\title{
Chemoselective Rhodium-Catalyzed Borylation of Bromoiodoarenes Under Mild Conditions
}

Anthony J. Varni, ${ }^{*}$ Michael V. Bautista, ${ }^{*}$ Kevin J. T. Noonan*

4400 Fifth Ave, Department of Chemistry, Carnegie Mellon University, Pittsburgh, Pennsylvania $15213-2617$

Corresponding Author Email Address: noonan@andrew.cmu.edu

$$
\text { Supporting Information }
$$

\section{Table of Contents}

GC-MS Analysis

Palladium-Catalyzed Borylation of 2-bromo-5-iodothiophene

Figure S1. GC-MS Conversion Data at $60{ }^{\circ} \mathrm{C}$

NMR Spectra (Figures S2-S23)

HRMS Spectra (Figures S24-S41)

GC-MS Spectra from Conversion Experiments (Figures S42-S64) S52-S74 


\section{GC-MS Analysis.}

Conversion was calculated using the equation below.

$$
\text { Percent Conversion }=\left(1-\frac{\text { Substrate at } t=24 h}{\text { Substrate at } t=0 \mathrm{~h}}\right) x\left(\frac{\text { Desired Product at } t=24 \mathrm{~h}}{\text { All Products Formed at } t=24 \mathrm{~h}}\right) x 100
$$

Palladium-Catalyzed Borylation of 2-bromo-5-iodothiophene.

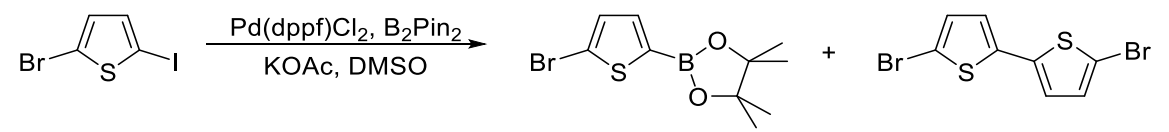

In a $\mathrm{N}_{2}$ filled glovebox, a $20 \mathrm{~mL}$ scintillation vial equipped with a Teflon screw cap was charged with an 2-bromo-5-iodothiophene (144 mg, $0.5 \mathrm{mmol}), \mathrm{Pd}(\mathrm{dppf}) \mathrm{Cl}_{2}(11 \mathrm{mg}, 0.015 \mathrm{mmol}), \mathrm{KOAc}$ (147 mg, $1.5 \mathrm{mmol}), \mathrm{B}_{2} \operatorname{pin}_{2}(121 \mathrm{mg}, 0.48 \mathrm{mmol})$, trimethoxybenzene (29 mg, $\left.0.17 \mathrm{mmol}\right)$, and $2.5 \mathrm{~mL}$ dry DMSO. The reaction vial was capped, removed from the glovebox, and placed in an oil bath at the specified temperature. The reaction was stirred for $24 \mathrm{~h}$, at which point, an aliquot was removed from the reaction mixture and conversion was determined using GC-MS analysis.

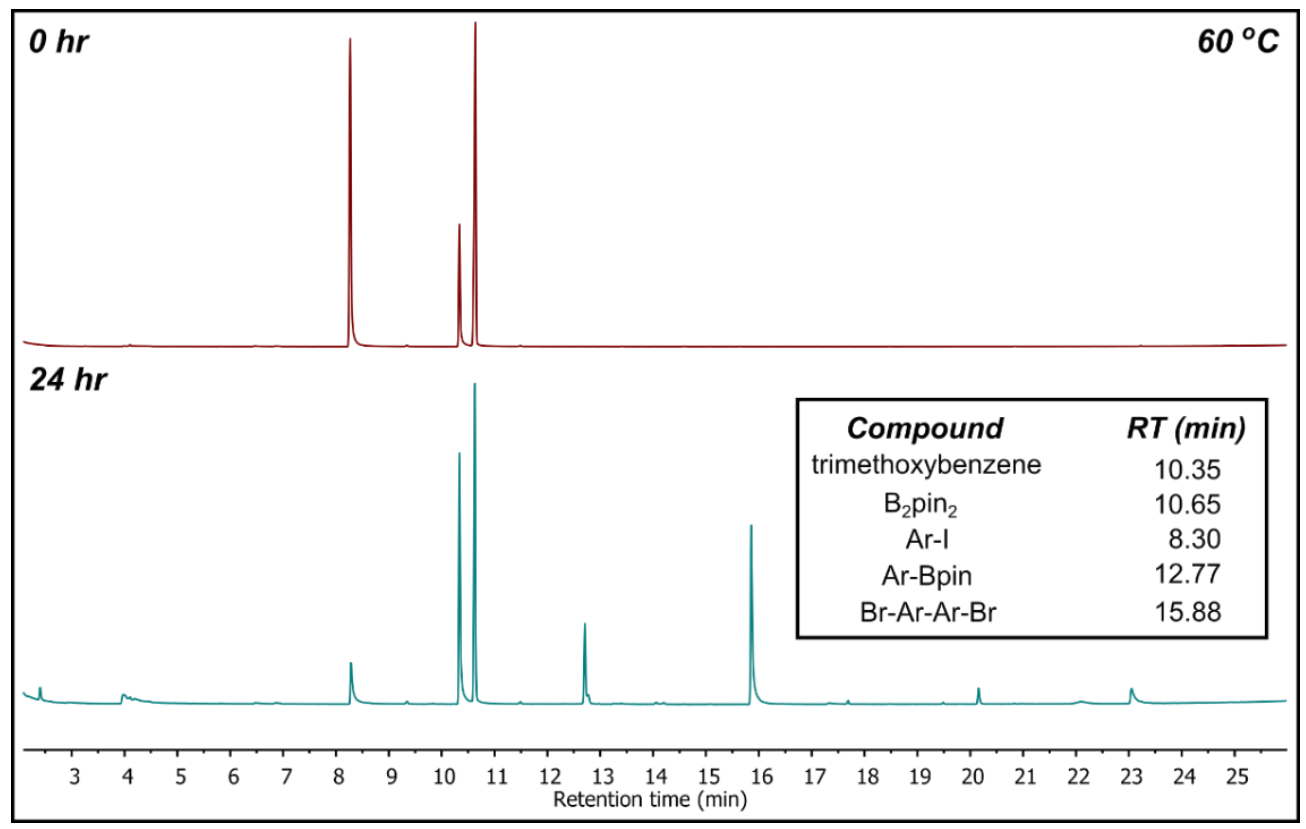

Figure S1. GC-MS spectra for conversion of 2-bromo-5-iodothiophene to 2-(5-bromothiophen-

2-yl)-4,4,5,5-tetramethyl-1,3,2-dioxaborolane at $60{ }^{\circ} \mathrm{C}$ using $\mathrm{Pd}(\mathrm{dppf}) \mathrm{Cl}_{2}$ as the catalyst. 


\section{NMR Spectra}

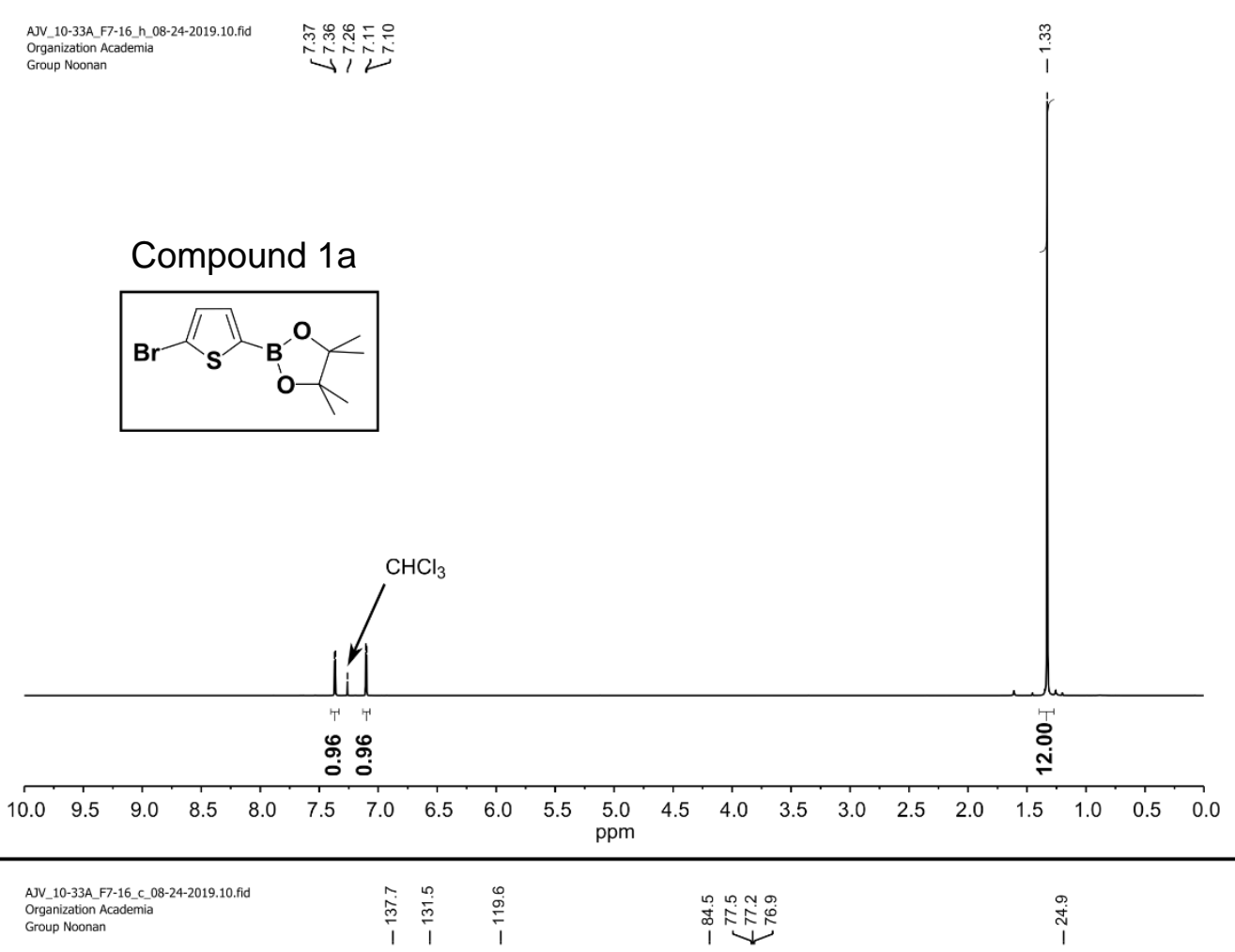

Compound 1a
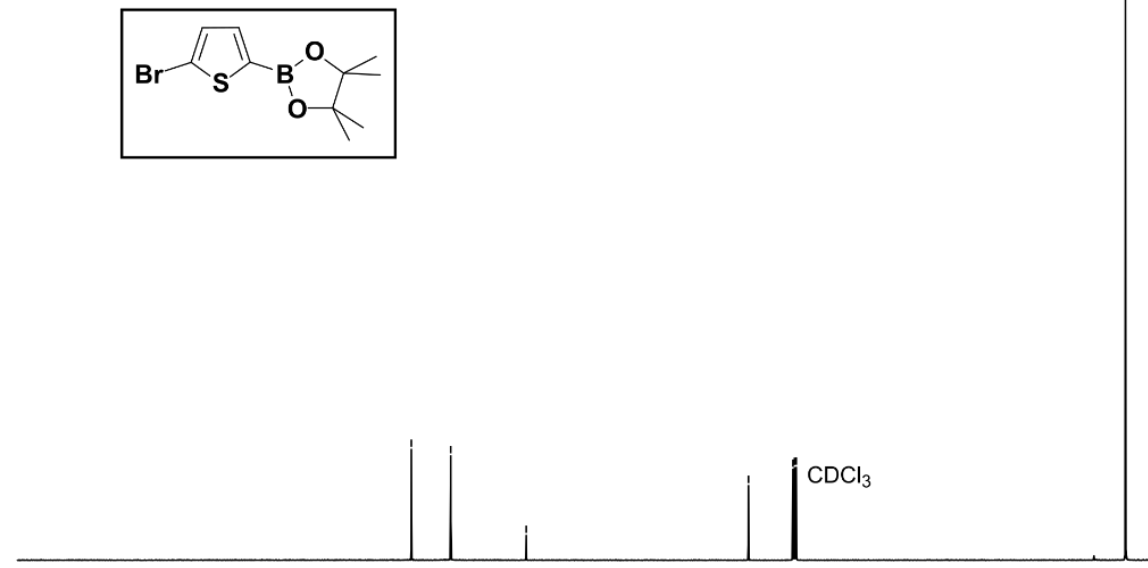

$\begin{array}{lllllllllllllllllllllll}200 & 190 & 180 & 170 & 160 & 150 & 140 & 130 & 120 & 110 & \begin{array}{c}100 \\ \mathrm{ppm}\end{array} & 90 & 80 & 70 & 60 & 50 & 40 & 30 & 20 & 10 & 0\end{array}$

Figure S2a. ${ }^{1} \mathrm{H}$ NMR spectrum (Top, $500 \mathrm{MHz}, \mathrm{CDCl}_{3}$ ) and ${ }^{13} \mathrm{C}\left\{{ }^{1} \mathrm{H}\right\} \mathrm{NMR}$ spectrum (Bottom, $126 \mathrm{MHz}_{\mathrm{CDCl}}$ ) of 2-(5-bromothiophen-2-yl)-4,4,5,5-tetramethyl-1,3,2-dioxaborolane. 


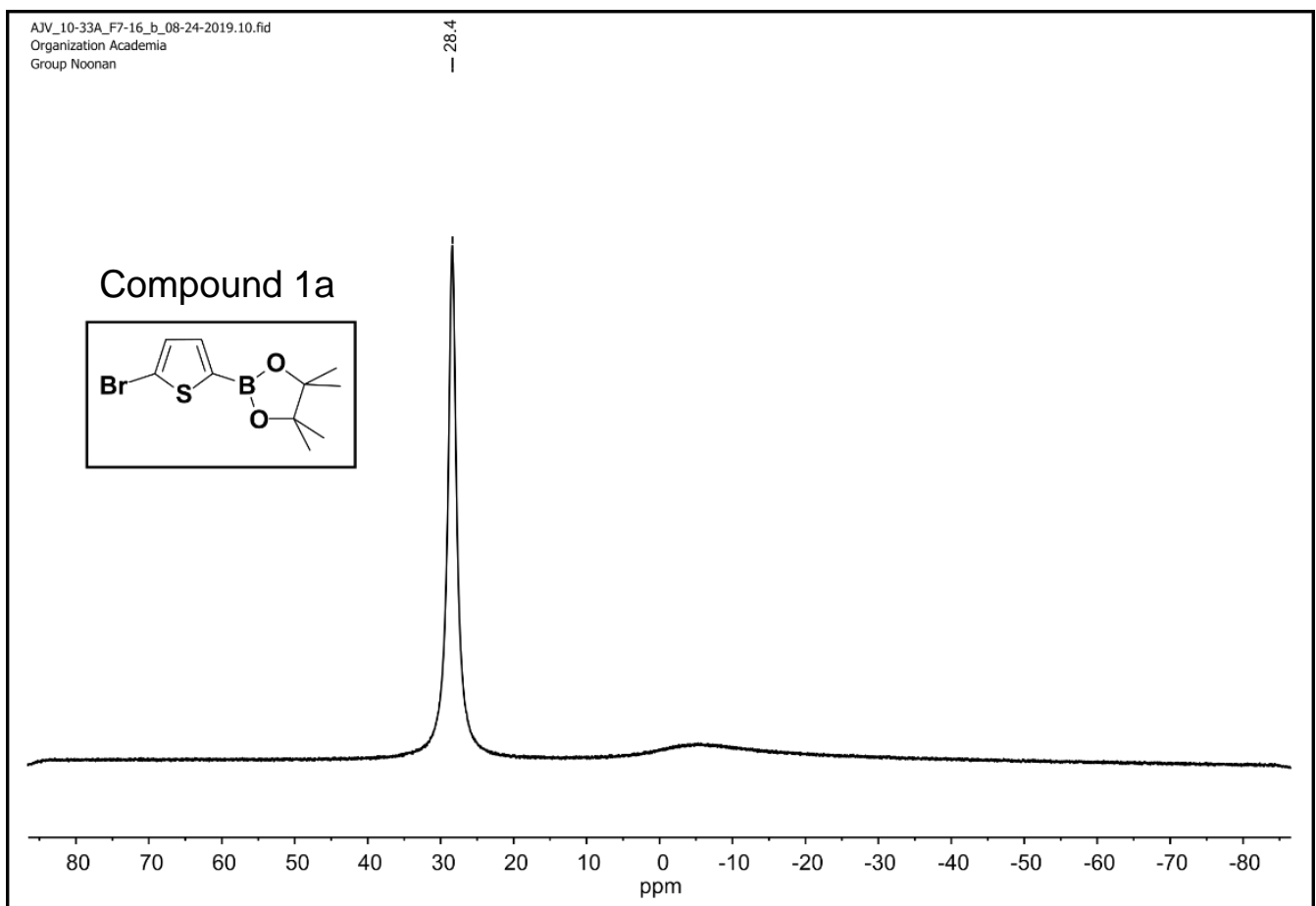

Figure S2b. ${ }^{11} \mathrm{~B}\left\{{ }^{1} \mathrm{H}\right\}$ NMR spectrum $\left(160 \mathrm{MHz}, \mathrm{CDCl}_{3}\right)$ of 2-(5-bromothiophen-2-yl)-4,4,5,5tetramethyl-1,3,2-dioxaborolane. 


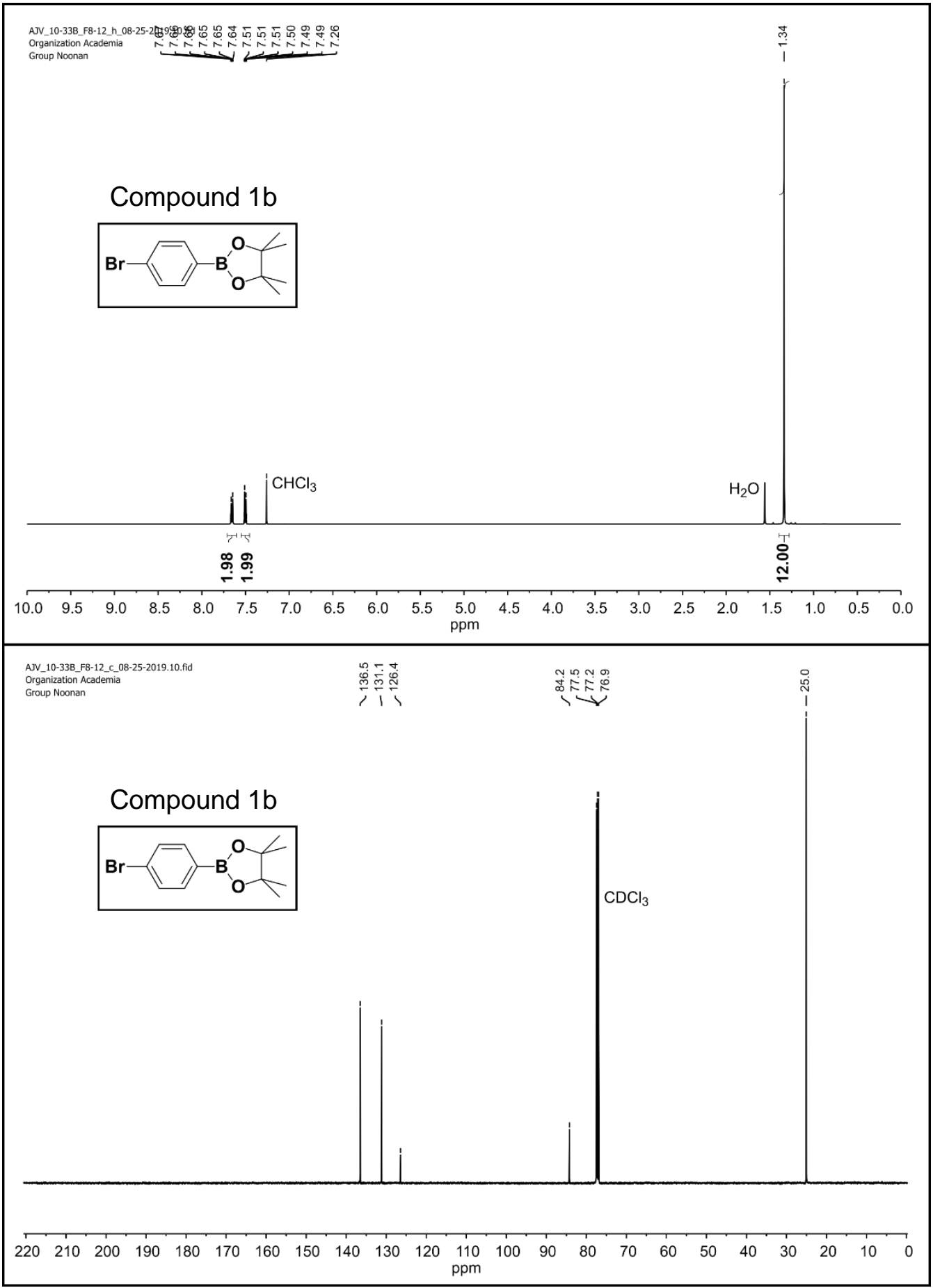

Figure S3a. ${ }^{1} \mathrm{H}$ NMR spectrum (Top, $500 \mathrm{MHz}, \mathrm{CDCl}_{3}$ ) and ${ }^{13} \mathrm{C}\left\{{ }^{1} \mathrm{H}\right\} \mathrm{NMR}$ spectrum (Bottom, $\left.126 \mathrm{MHz}, \mathrm{CDCl}_{3}\right)$ of 2-(4-bromophenyl)-4,4,5,5-tetramethyl-1,3,2-dioxaborolane. 


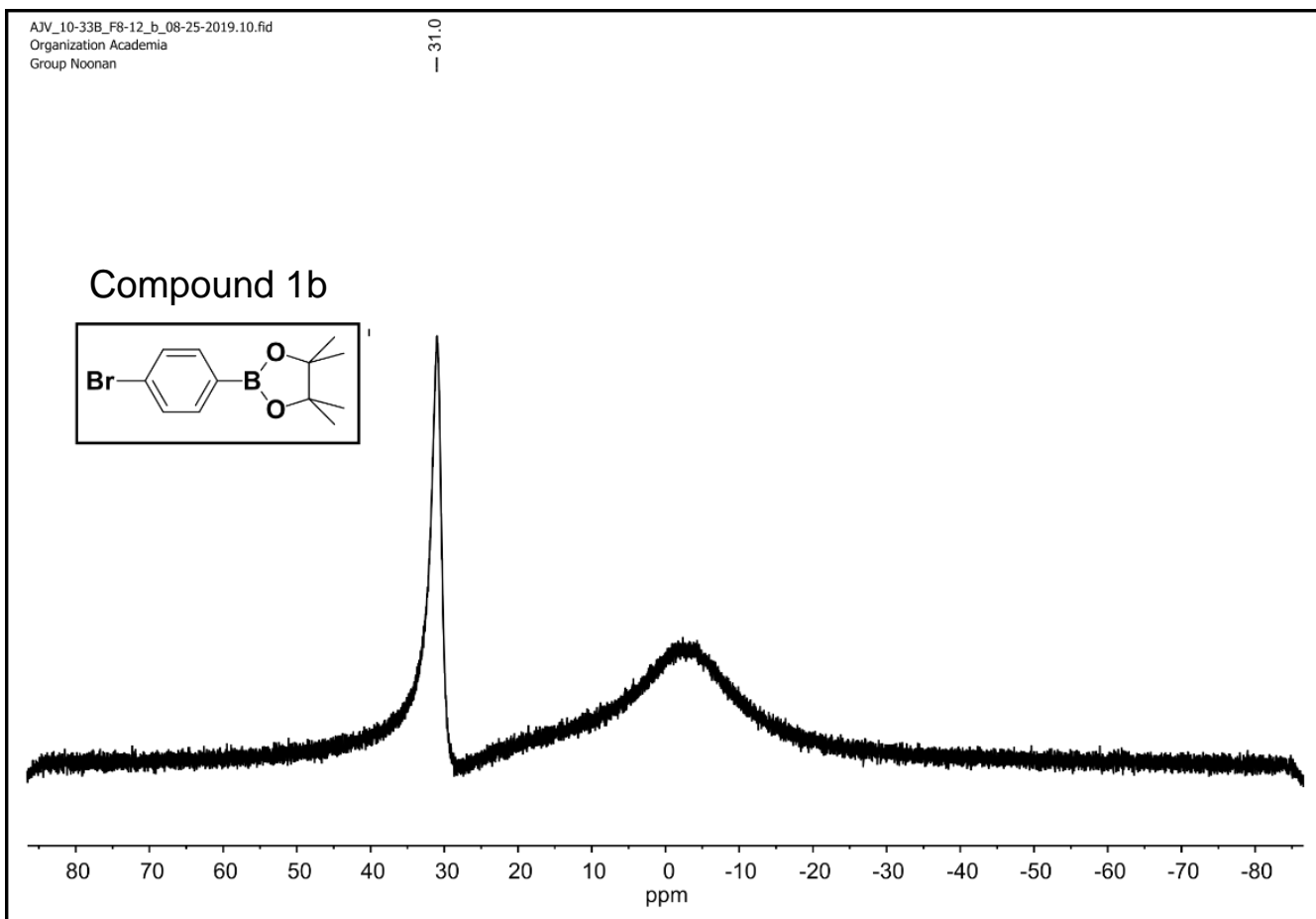

Figure S3b. ${ }^{11} \mathrm{~B}\left\{{ }^{1} \mathrm{H}\right\}$ NMR spectrum (160 $\left.\mathrm{MHz}, \mathrm{CDCl}_{3}\right)$ of 2-(4-bromophenyl)-4,4,5,5tetramethyl-1,3,2-dioxaborolane. 


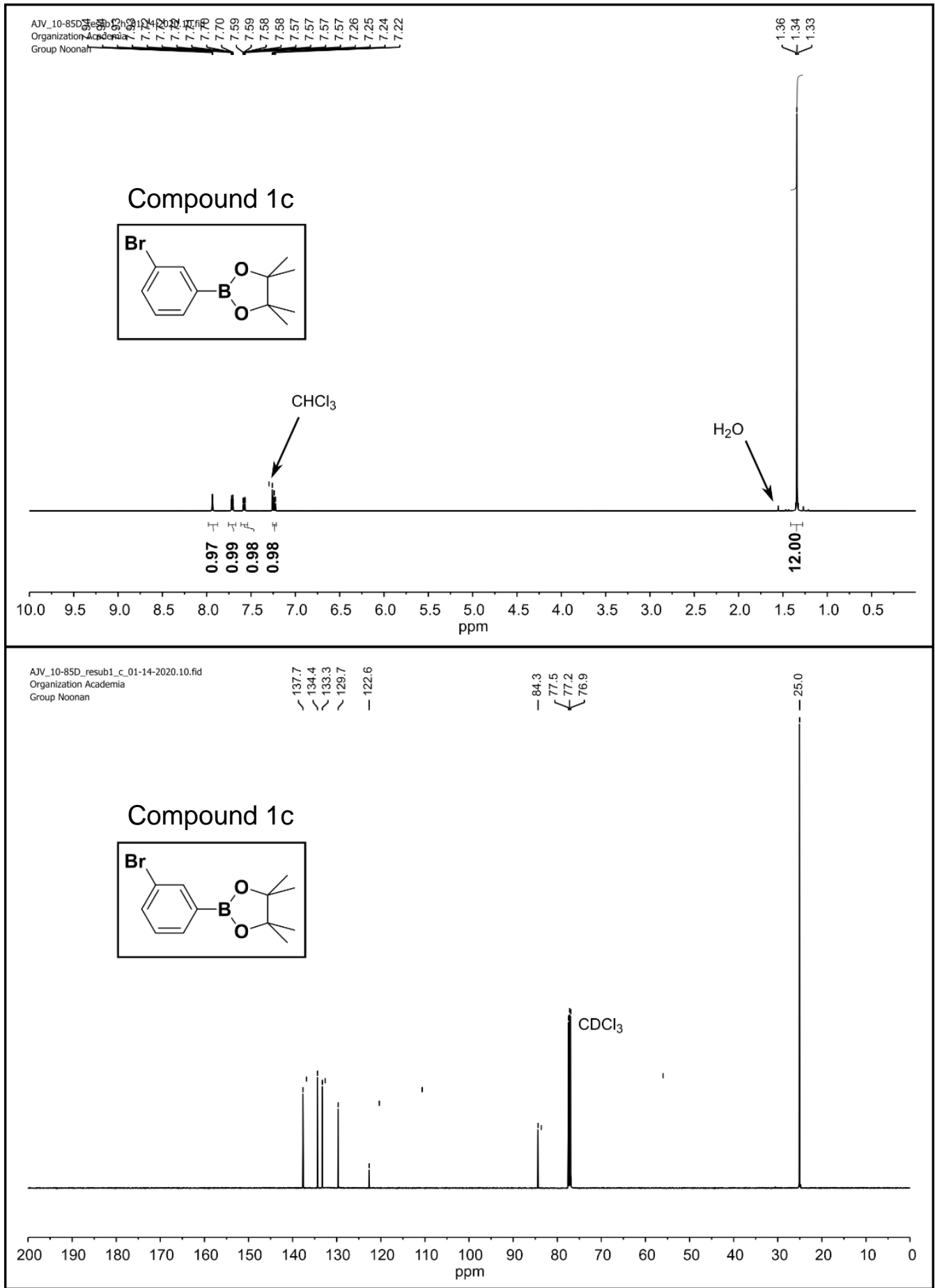

Figure S4a. ${ }^{1} \mathrm{H}$ NMR spectrum (Top, $500 \mathrm{MHz}, \mathrm{CDCl}_{3}$ ) and ${ }^{13} \mathrm{C}\left\{{ }^{1} \mathrm{H}\right\} \mathrm{NMR}$ spectrum (Bottom, $\left.126 \mathrm{MHz}, \mathrm{CDCl}_{3}\right)$ of 2-(3-bromophenyl)-4,4,5,5-tetramethyl-1,3,2-dioxaborolane. 


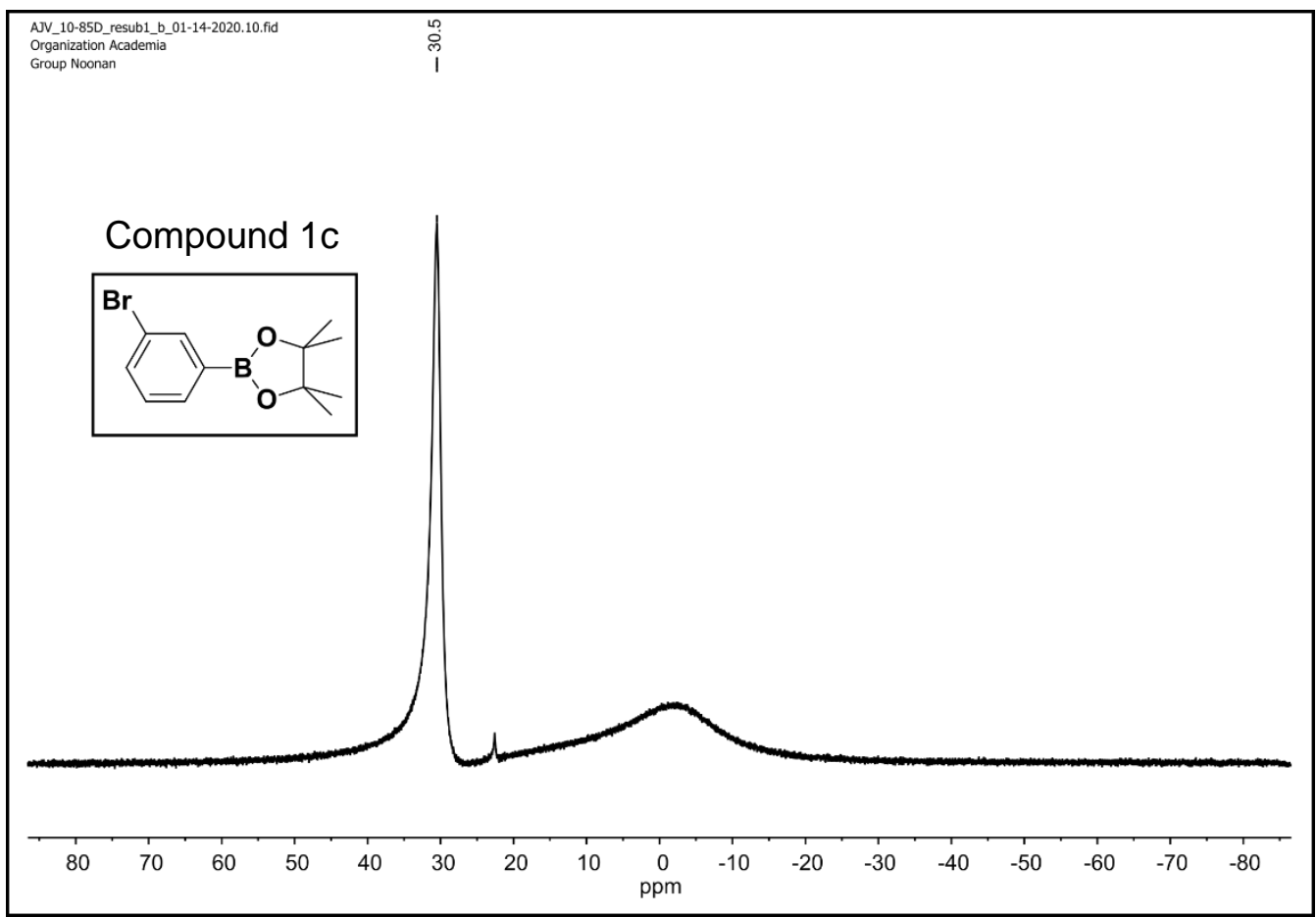

Figure S4b. ${ }^{11} \mathrm{~B}\left\{{ }^{1} \mathrm{H}\right\}$ NMR spectrum (160 $\left.\mathrm{MHz}, \mathrm{CDCl}_{3}\right)$ of 2-(3-bromophenyl)-4,4,5,5tetramethyl-1,3,2-dioxaborolane. 


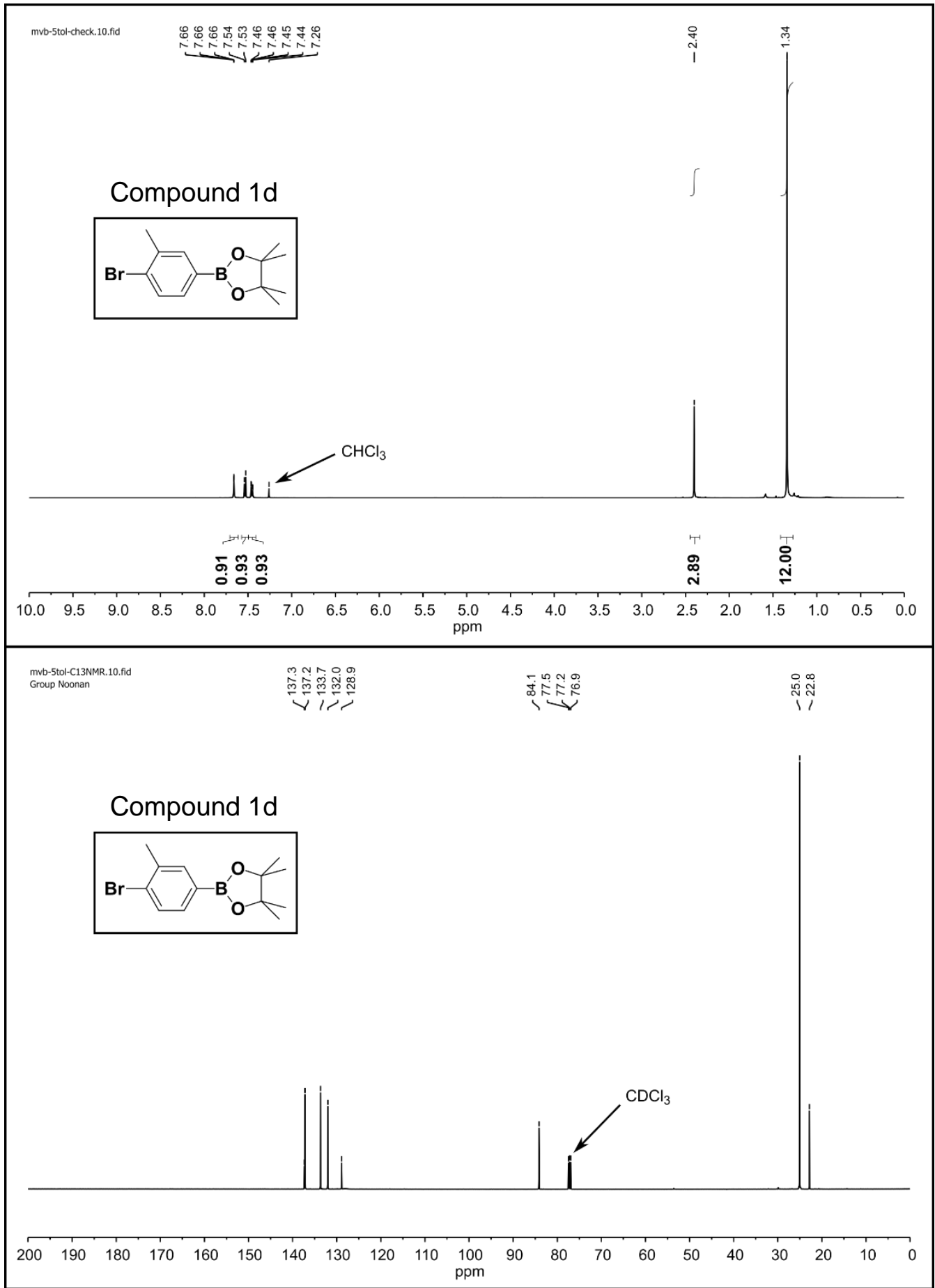

Figure S5a. ${ }^{1} \mathrm{H}$ NMR spectrum (Top, $500 \mathrm{MHz}, \mathrm{CDCl}_{3}$ ) and ${ }^{13} \mathrm{C}\left\{{ }^{1} \mathrm{H}\right\}$ NMR spectrum (Bottom, $\left.126 \mathrm{MHz}, \mathrm{CDCl}_{3}\right)$ of 2-(4-bromo-3-methylphenyl)-4,4,5,5-tetramethyl-1,3,2-dioxaborolane. 


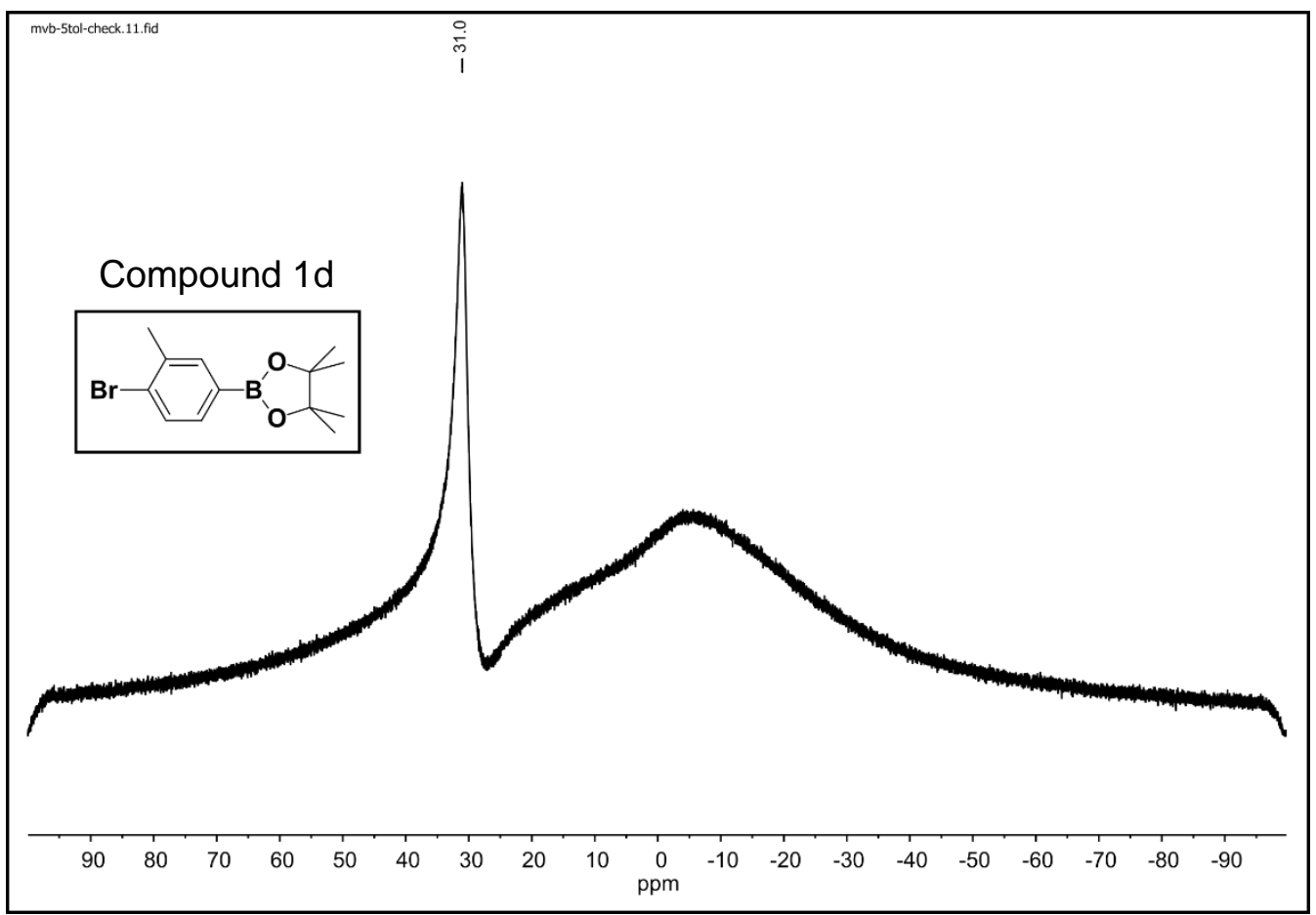

Figure S5b. ${ }^{11} \mathrm{~B}\left\{{ }^{1} \mathrm{H}\right\}$ NMR spectrum (160 $\left.\mathrm{MHz}, \mathrm{CDCl}_{3}\right)$ of 2-(4-bromo-3-methylphenyl)4,4,5,5-tetramethyl-1,3,2-dioxaborolane. 


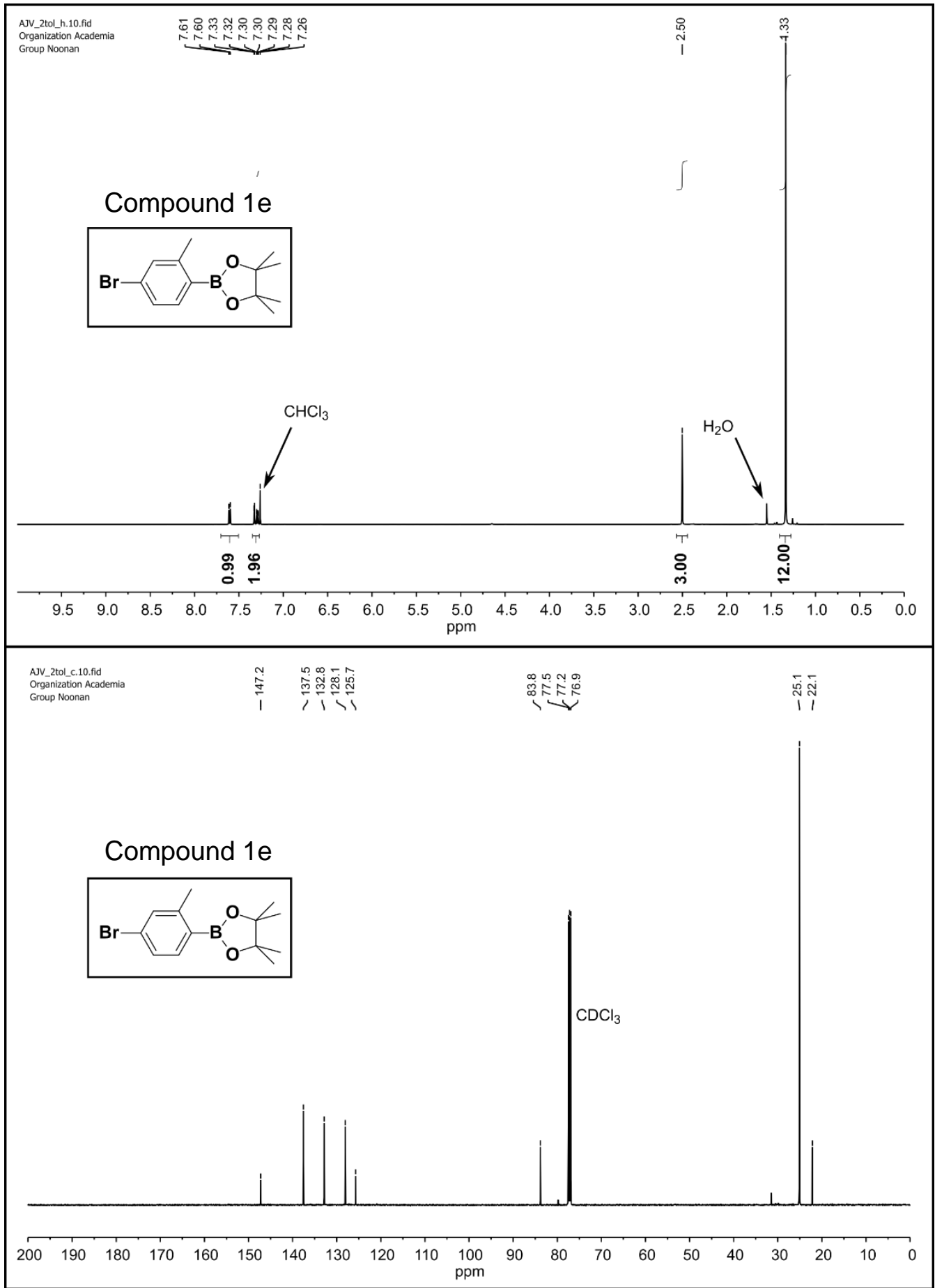

Figure S6a. ${ }^{1} \mathrm{H}$ NMR spectrum (Top, $500 \mathrm{MHz}, \mathrm{CDCl}_{3}$ ) and ${ }^{13} \mathrm{C}\left\{{ }^{1} \mathrm{H}\right\}$ NMR spectrum (Bottom, $126 \mathrm{MHz}_{\mathrm{CDCl}}$ ) of 2-(4-bromo-2-methylphenyl)-4,4,5,5-tetramethyl-1,3,2-dioxaborolane. 


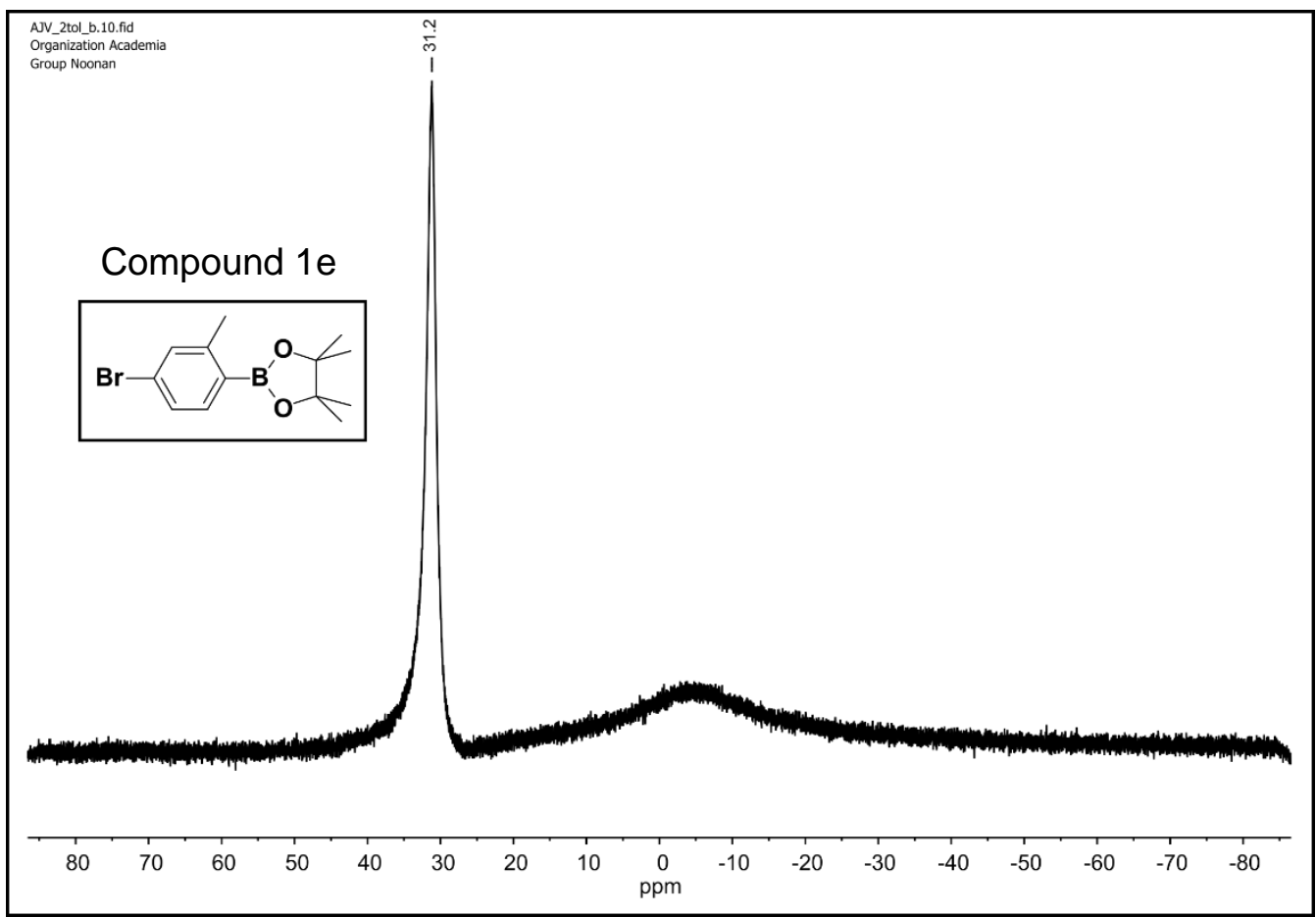

Figure S6b. ${ }^{11} \mathrm{~B}\left\{{ }^{1} \mathrm{H}\right\}$ NMR spectrum (160 $\left.\mathrm{MHz}, \mathrm{CDCl}_{3}\right)$ of 2-(4-bromo-2-methylphenyl)4,4,5,5-tetramethyl-1,3,2-dioxaborolane. 


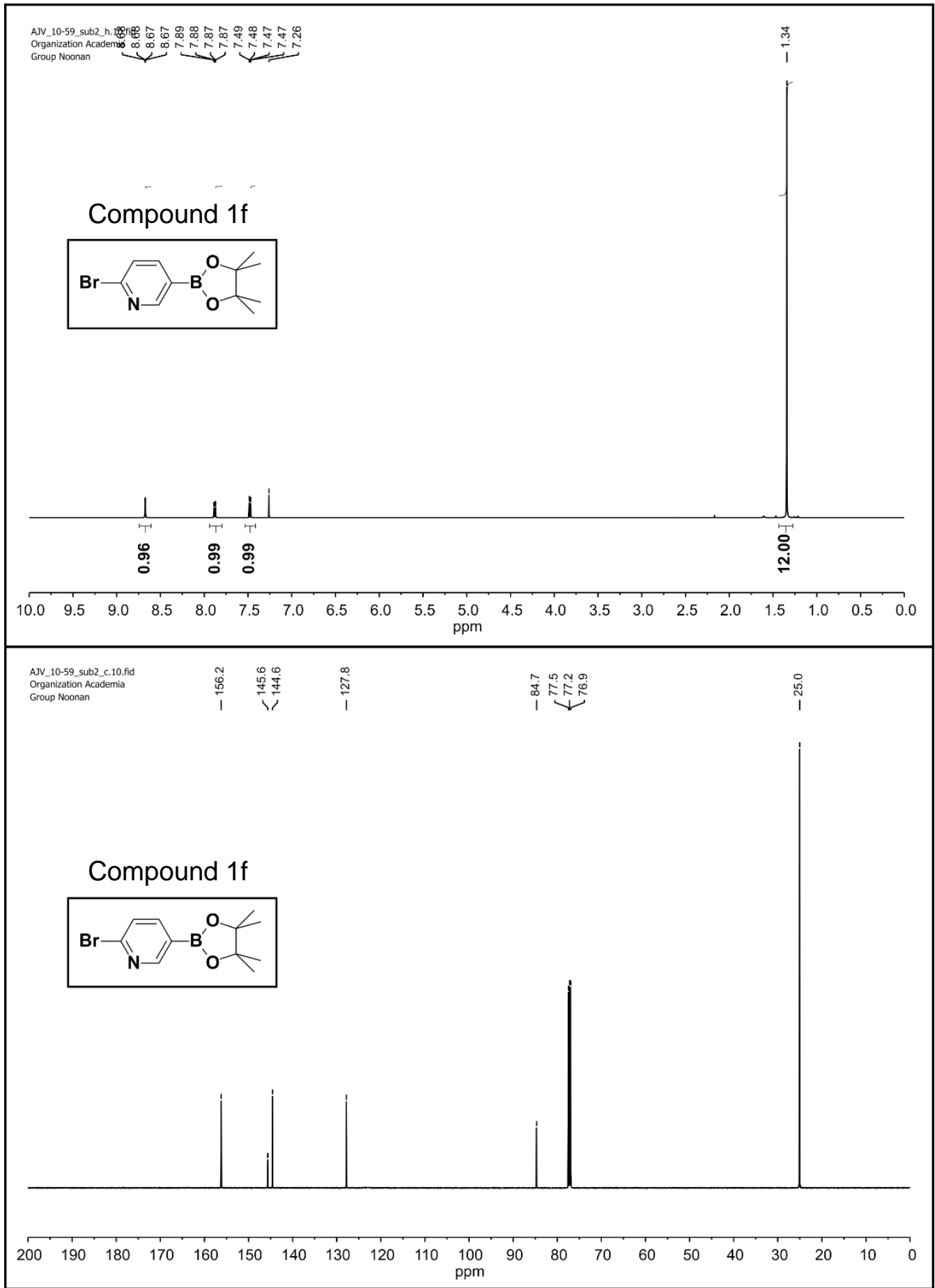

Figure S7a. ${ }^{1} \mathrm{H}$ NMR spectrum (Top, $500 \mathrm{MHz}, \mathrm{CDCl}_{3}$ ) and ${ }^{13} \mathrm{C}\left\{{ }^{1} \mathrm{H}\right\} \mathrm{NMR}$ spectrum (Bottom, $\left.126 \mathrm{MHz}_{\mathrm{CDCl}}\right)$ of 2-bromo-5-(4,4,5,5-tetramethyl-1,3,2-dioxaborolan-2-yl)pyridine. 


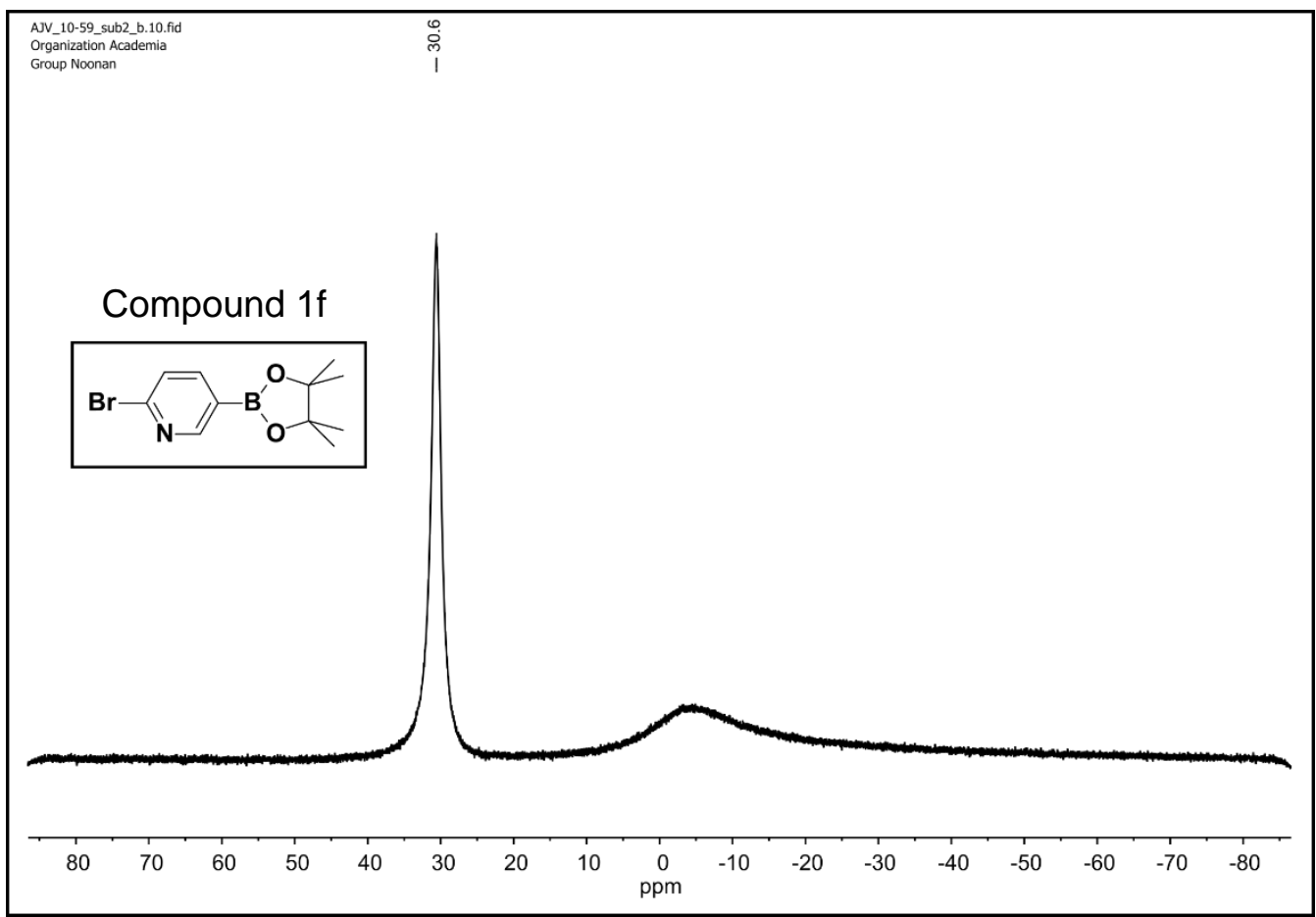

Figure S7b. ${ }^{11} \mathrm{~B}\left\{{ }^{1} \mathrm{H}\right\}$ NMR spectrum $\left(160 \mathrm{MHz}, \mathrm{CDCl}_{3}\right)$ of 2-bromo-5-(4,4,5,5-tetramethyl1,3,2-dioxaborolan-2-yl)pyridine. 


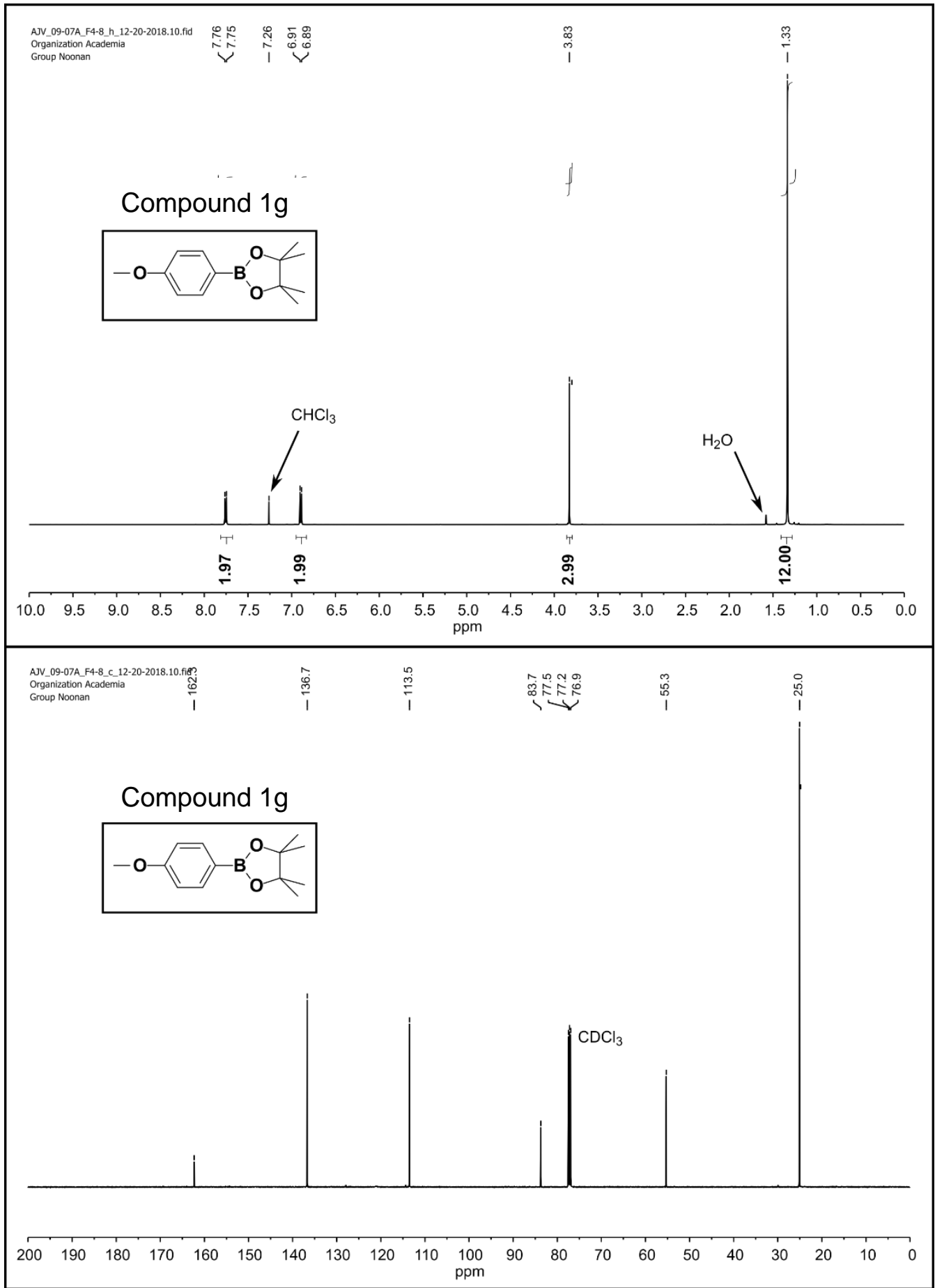

Figure S8a. ${ }^{1} \mathrm{H}$ NMR spectrum (Top, $500 \mathrm{MHz}, \mathrm{CDCl}_{3}$ ) and ${ }^{13} \mathrm{C}\left\{{ }^{1} \mathrm{H}\right\} \mathrm{NMR}$ spectrum (Bottom, $126 \mathrm{MHz}_{\mathrm{CDCl}}$ ) of 2-(4-methoxyphenyl)-4,4,5,5-tetramethyl-1,3,2-dioxaborolane. 


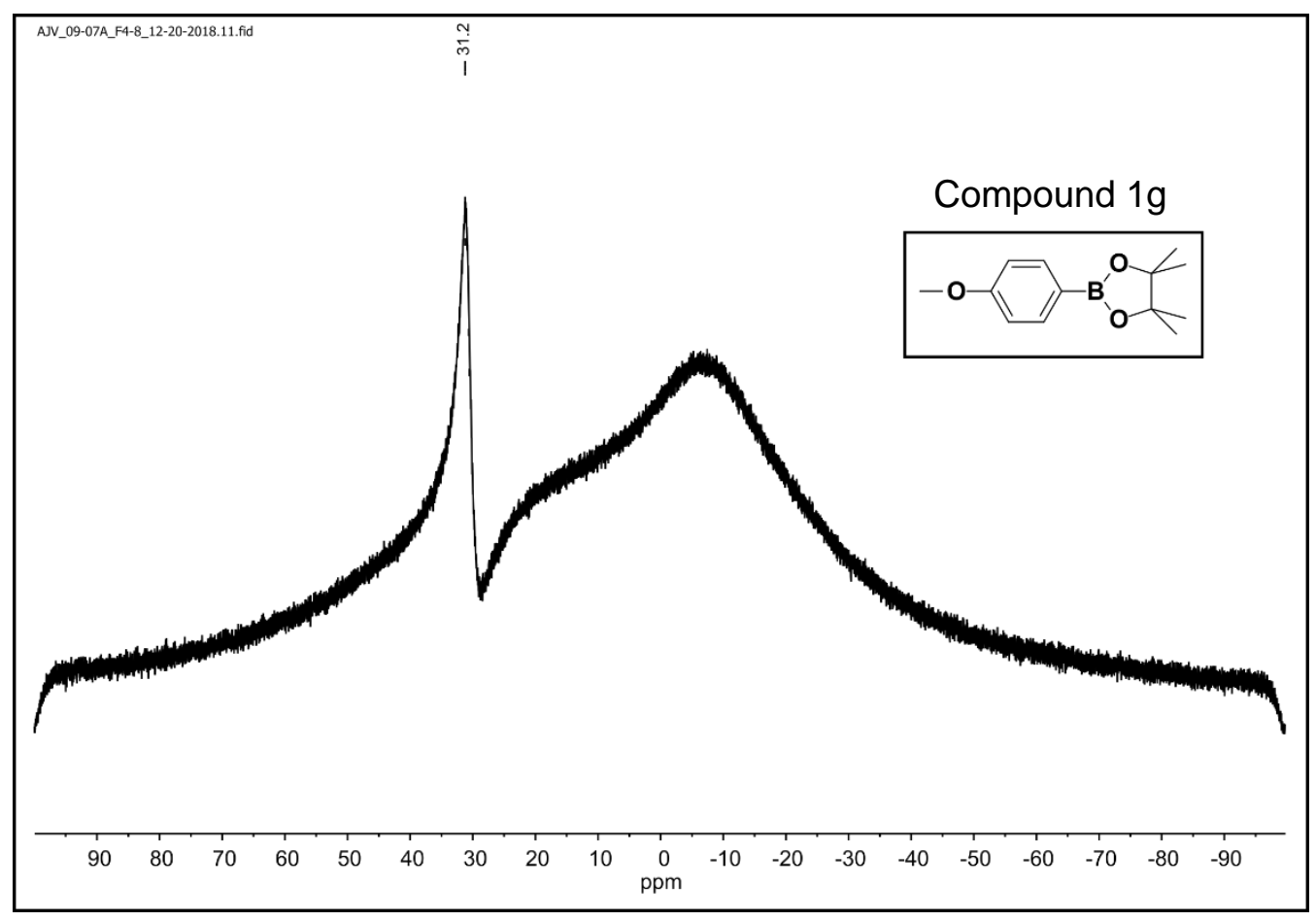

Figure S8b. ${ }^{11} \mathrm{~B}\left\{{ }^{1} \mathrm{H}\right\}$ NMR spectrum (160 $\left.\mathrm{MHz}, \mathrm{CDCl}_{3}\right)$ of 2-(4-methoxyphenyl)-4,4,5,5tetramethyl-1,3,2-dioxaborolane. 


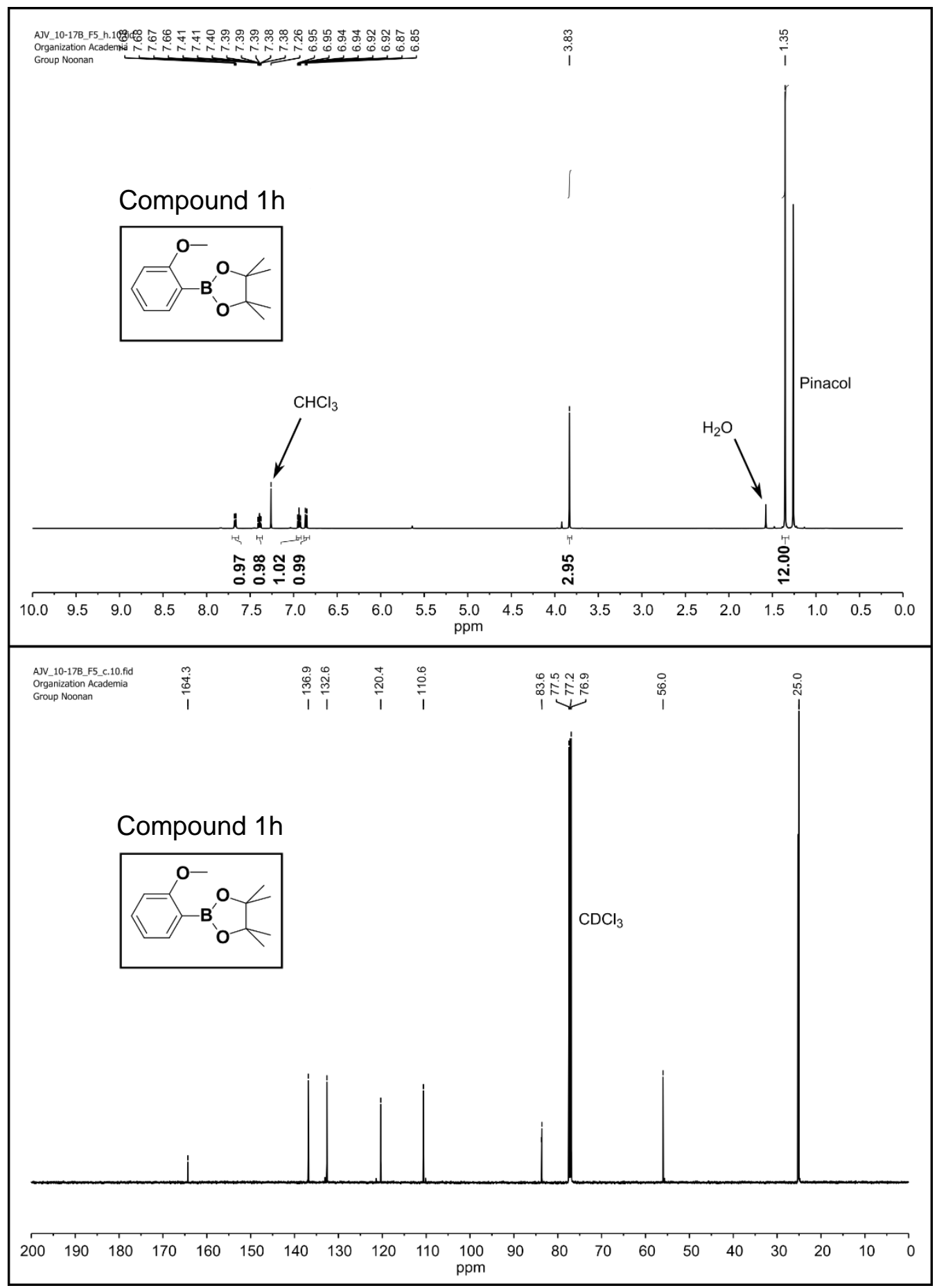

Figure S9a. ${ }^{1} \mathrm{H}$ NMR spectrum (Top, $500 \mathrm{MHz}, \mathrm{CDCl}_{3}$ ) and ${ }^{13} \mathrm{C}\left\{{ }^{1} \mathrm{H}\right\} \mathrm{NMR}$ spectrum (Bottom, $\left.126 \mathrm{MHz}, \mathrm{CDCl}_{3}\right)$ of 2-(2-methoxyphenyl)-4,4,5,5-tetramethyl-1,3,2-dioxaborolane. 


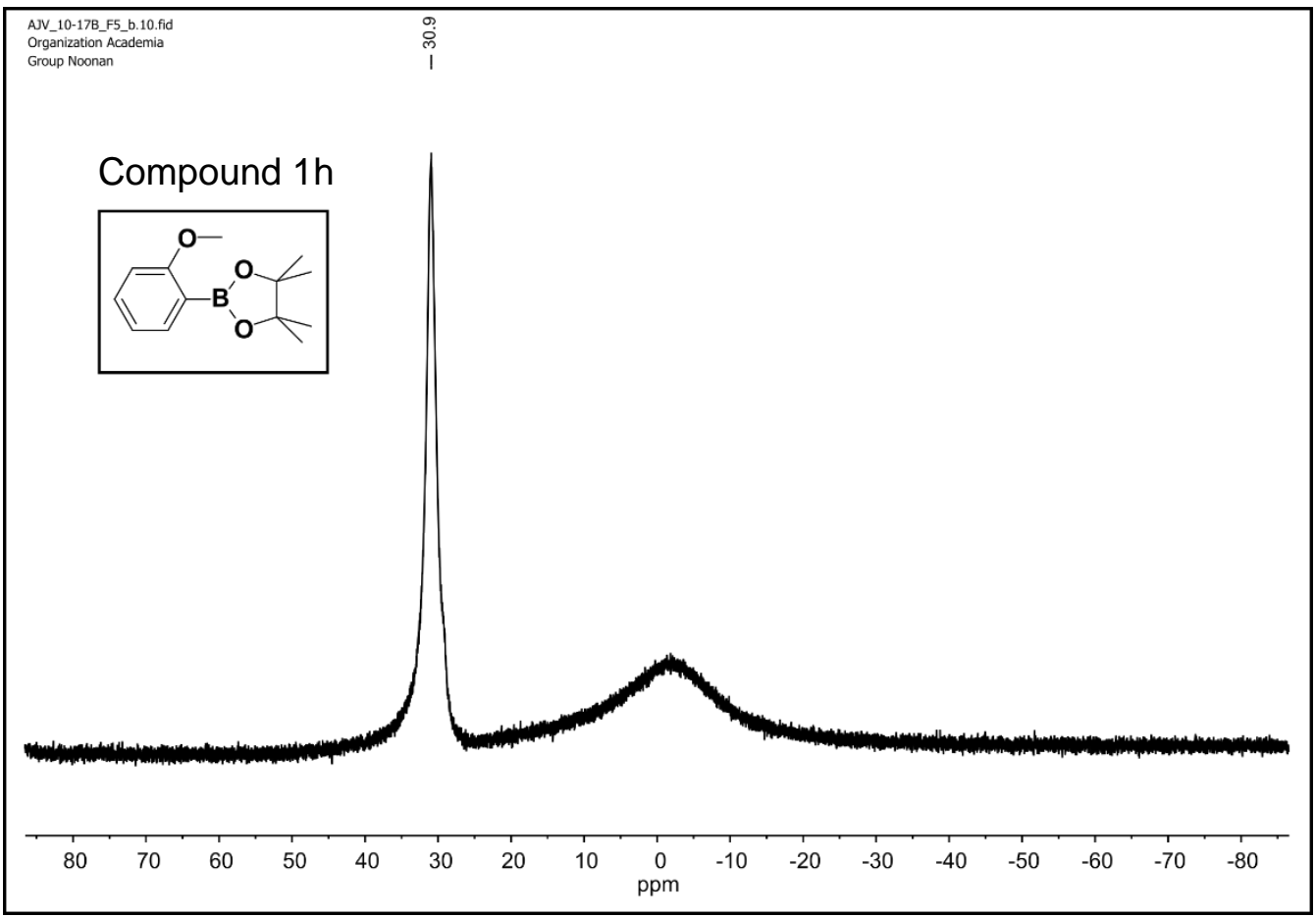

Figure S9b. ${ }^{11} \mathrm{~B}\left\{{ }^{1} \mathrm{H}\right\}$ NMR spectrum (160 $\left.\mathrm{MHz}, \mathrm{CDCl}_{3}\right)$ of 2-(2-methoxyphenyl)-4,4,5,5tetramethyl-1,3,2-dioxaborolane. 


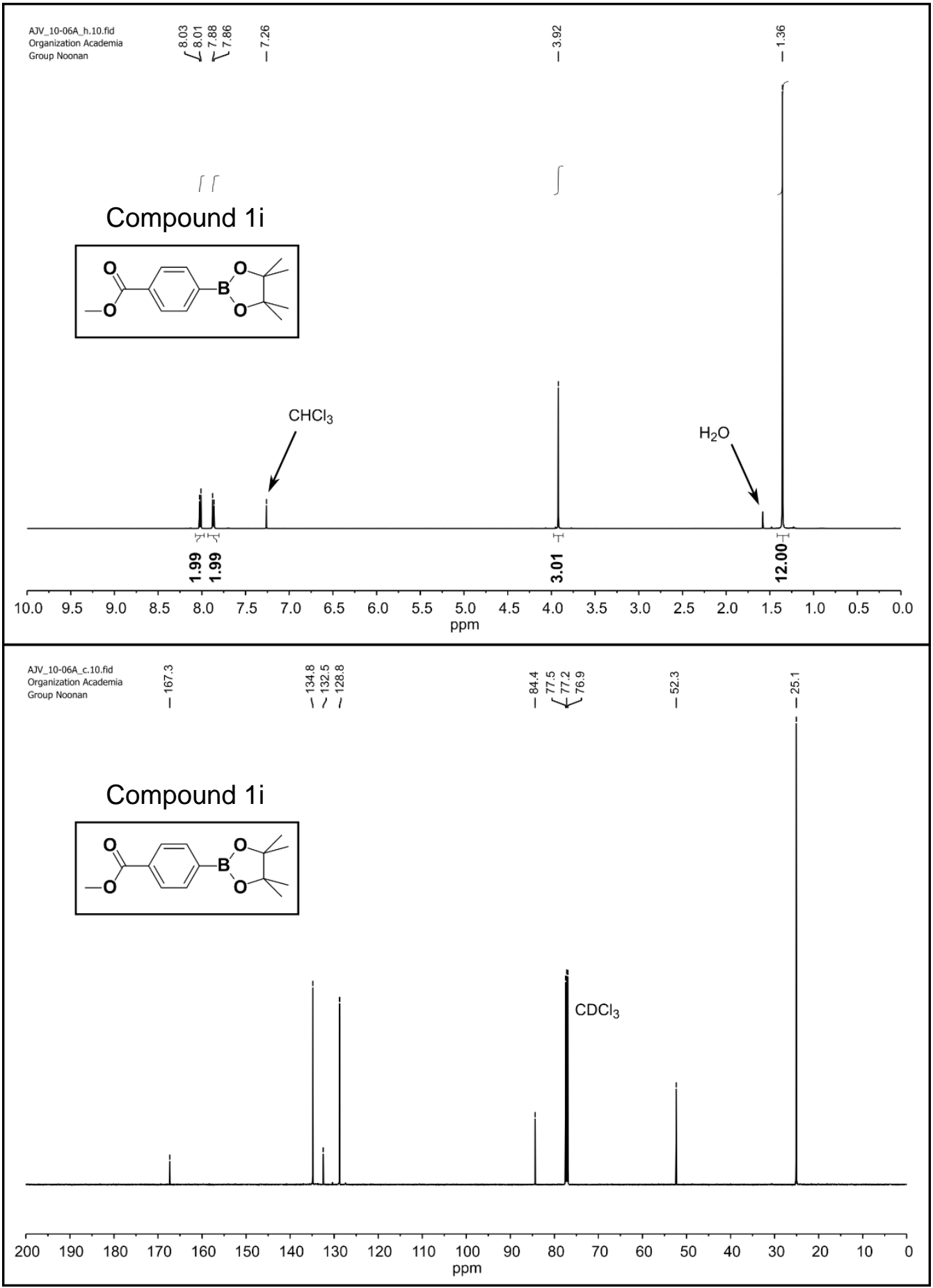

Figure S10a. ${ }^{1} \mathrm{H}$ NMR spectrum (Top, $500 \mathrm{MHz}, \mathrm{CDCl}_{3}$ ) and ${ }^{13} \mathrm{C}\left\{{ }^{1} \mathrm{H}\right\} \mathrm{NMR}$ spectrum (Bottom, $\left.126 \mathrm{MHz}, \mathrm{CDCl}_{3}\right)$ of methyl 4-(4,4,5,5-tetramethyl-1,3,2-dioxaborolan-2-yl)benzoate. 


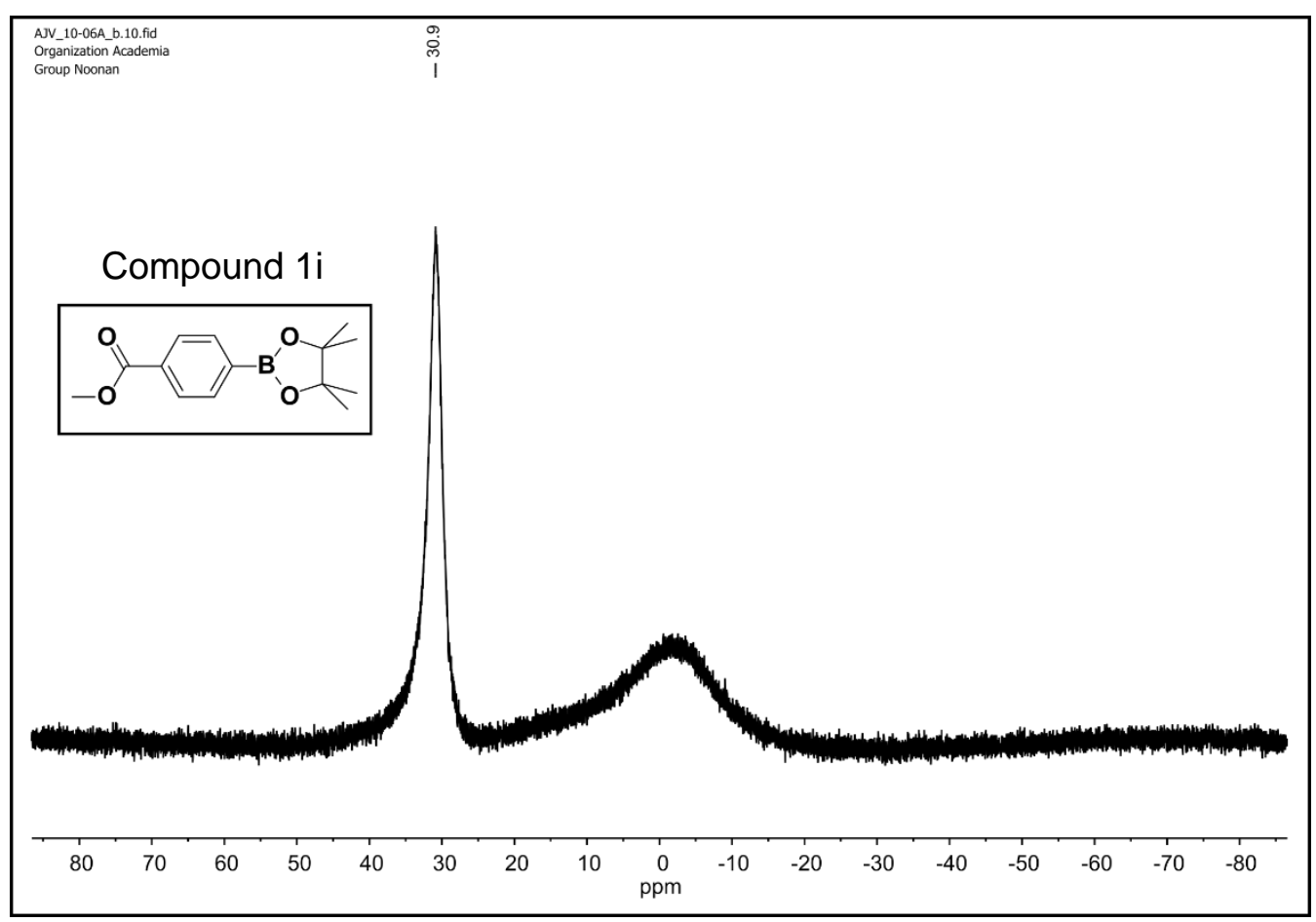

Figure S10b. ${ }^{11} \mathrm{~B}\left\{{ }^{1} \mathrm{H}\right\}$ NMR spectrum $\left(160 \mathrm{MHz}, \mathrm{CDCl}_{3}\right)$ of methyl 4-(4,4,5,5-tetramethyl1,3,2-dioxaborolan-2-yl)benzoate. 


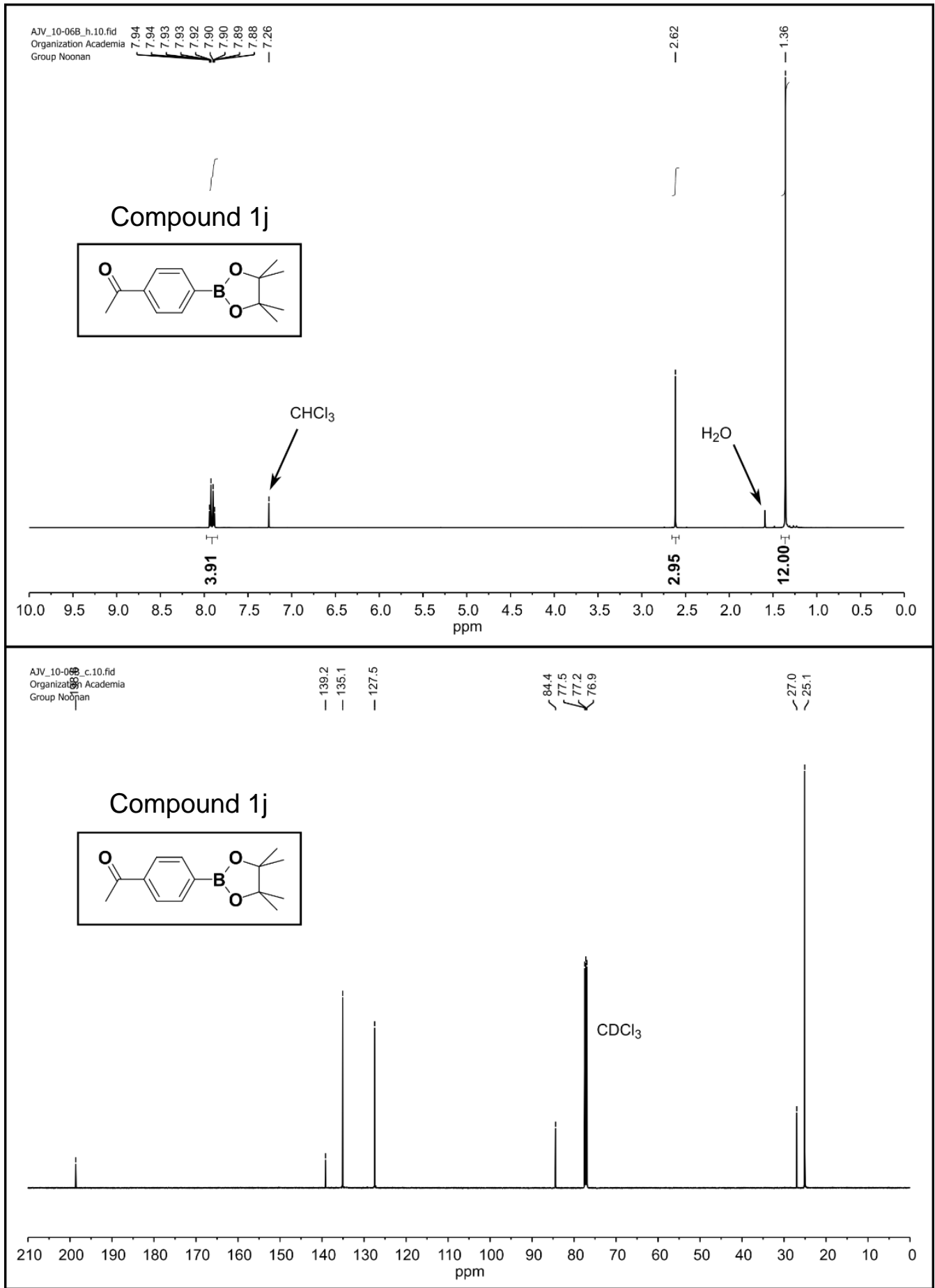

Figure S11a. ${ }^{1} \mathrm{H}$ NMR spectrum (Top, $500 \mathrm{MHz}, \mathrm{CDCl}_{3}$ ) and ${ }^{13} \mathrm{C}\left\{{ }^{1} \mathrm{H}\right\} \mathrm{NMR}$ spectrum (Bottom, $\left.126 \mathrm{MHz}, \mathrm{CDCl}_{3}\right)$ of 1-(4-(4,4,5,5-tetramethyl-1,3,2-dioxaborolan-2-yl)phenyl)ethan-1-one. 


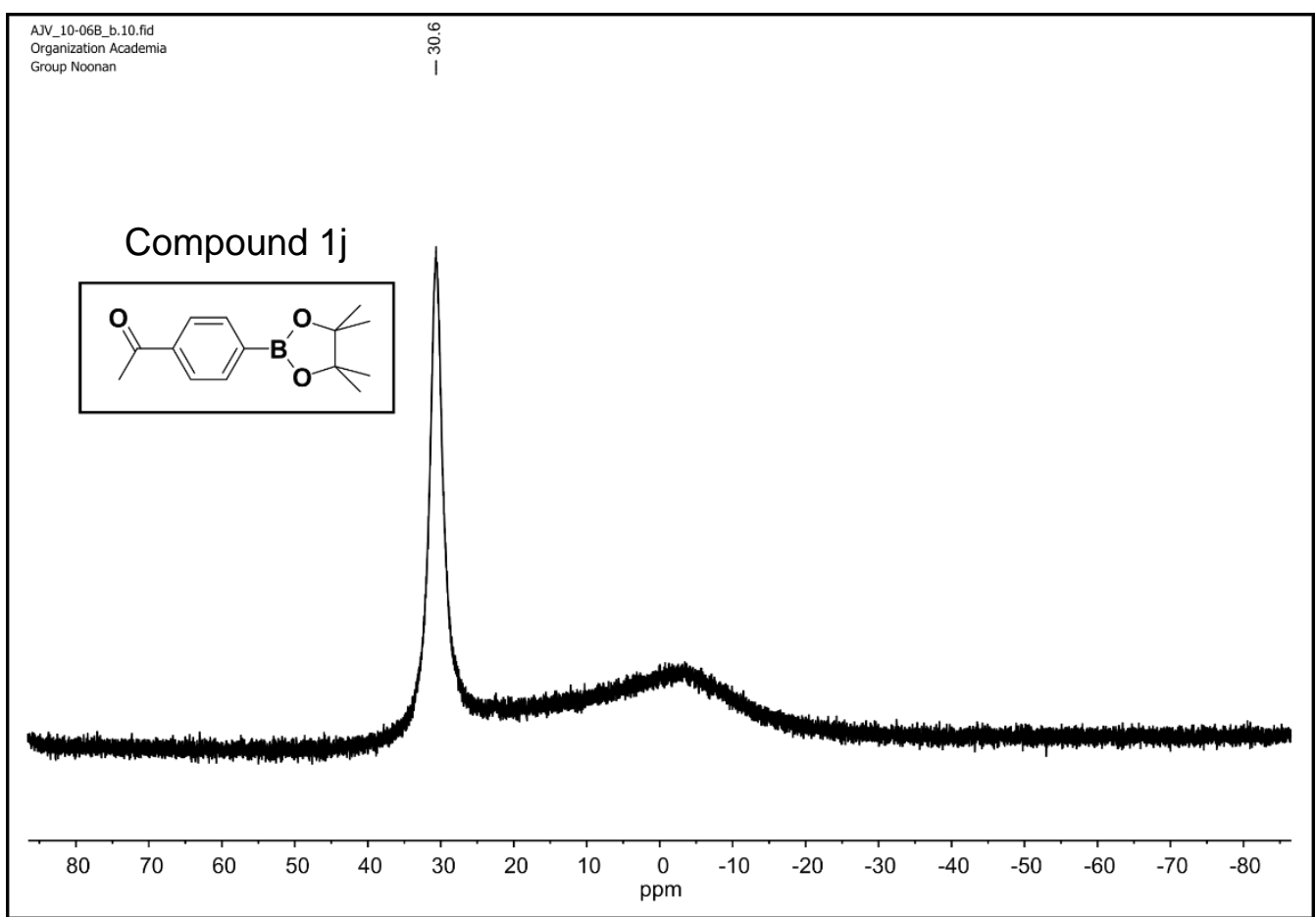

Figure S11b. ${ }^{11} \mathrm{~B}\left\{{ }^{1} \mathrm{H}\right\}$ NMR spectrum $\left(160 \mathrm{MHz}, \mathrm{CDCl}_{3}\right)$ of 1-(4-(4,4,5,5-tetramethyl-1,3,2dioxaborolan-2-yl)phenyl)ethan-1-one. 


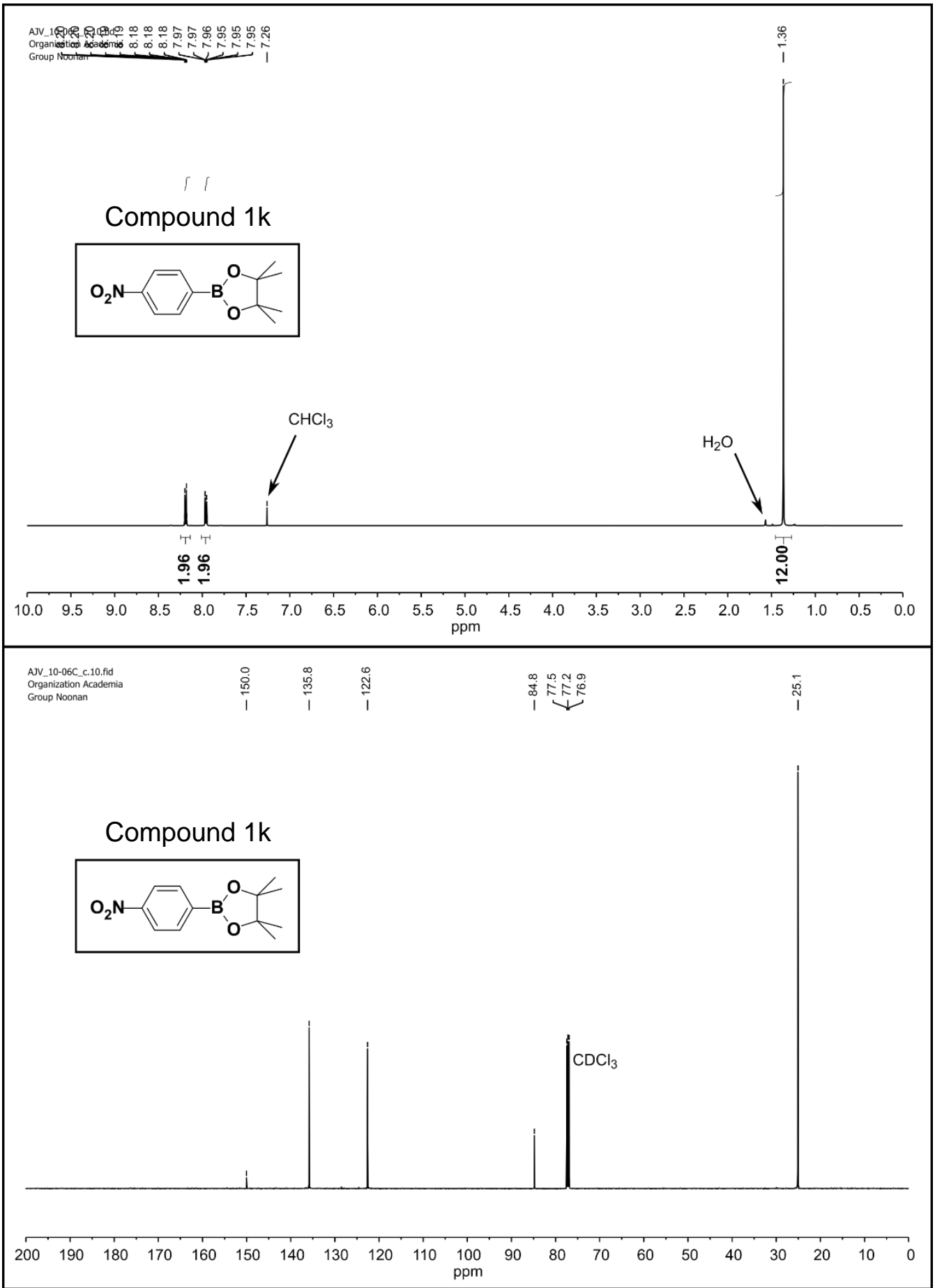

Figure S12a. ${ }^{1} \mathrm{H}$ NMR spectrum (Top, $500 \mathrm{MHz}, \mathrm{CDCl}_{3}$ ) and ${ }^{13} \mathrm{C}\left\{{ }^{1} \mathrm{H}\right\} \mathrm{NMR}$ spectrum (Bottom, $126 \mathrm{MHz}_{\mathrm{CDCl}}$ ) of 4,4,5,5-tetramethyl-2-(4-nitrophenyl)-1,3,2-dioxaborolane. 


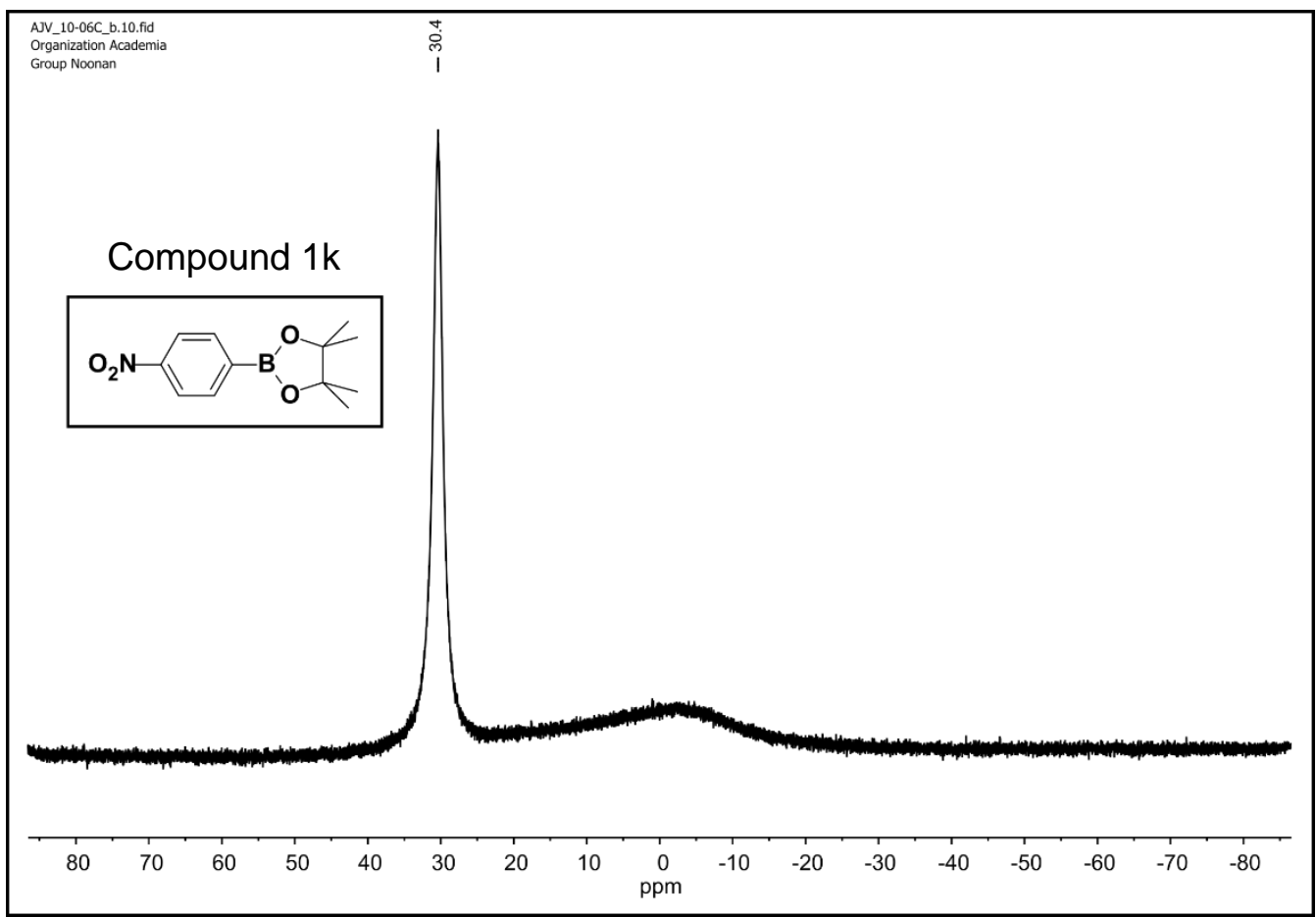

Figure S12b. ${ }^{11} \mathrm{~B}\left\{{ }^{1} \mathrm{H}\right\}$ NMR spectrum (160 $\left.\mathrm{MHz}, \mathrm{CDCl}_{3}\right)$ of 4,4,5,5-tetramethyl-2-(4nitrophenyl)-1,3,2-dioxaborolane. 


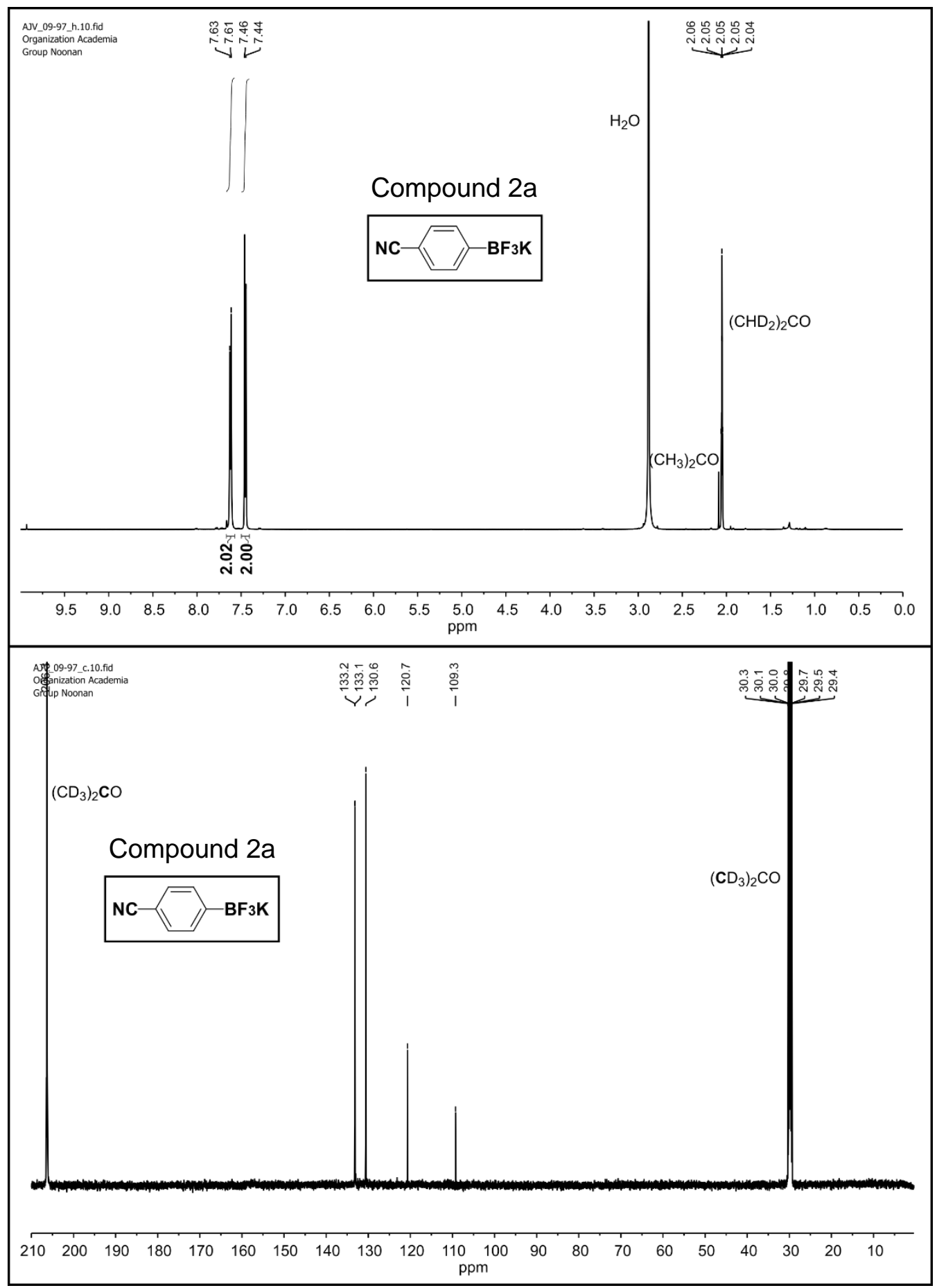

Figure S13a. ${ }^{1} \mathrm{H}$ NMR spectrum (Top, $500 \mathrm{MHz},\left(\mathrm{CD}_{3}\right)_{2} \mathrm{CO}$ ) and ${ }^{13} \mathrm{C}\left\{{ }^{1} \mathrm{H}\right\} \mathrm{NMR}$ spectrum (Bottom, $126 \mathrm{MHz},\left(\mathrm{CD}_{3}\right)_{2} \mathrm{CO}$ ) of potassium (4-cyanophenyl)trifluoroborate. 


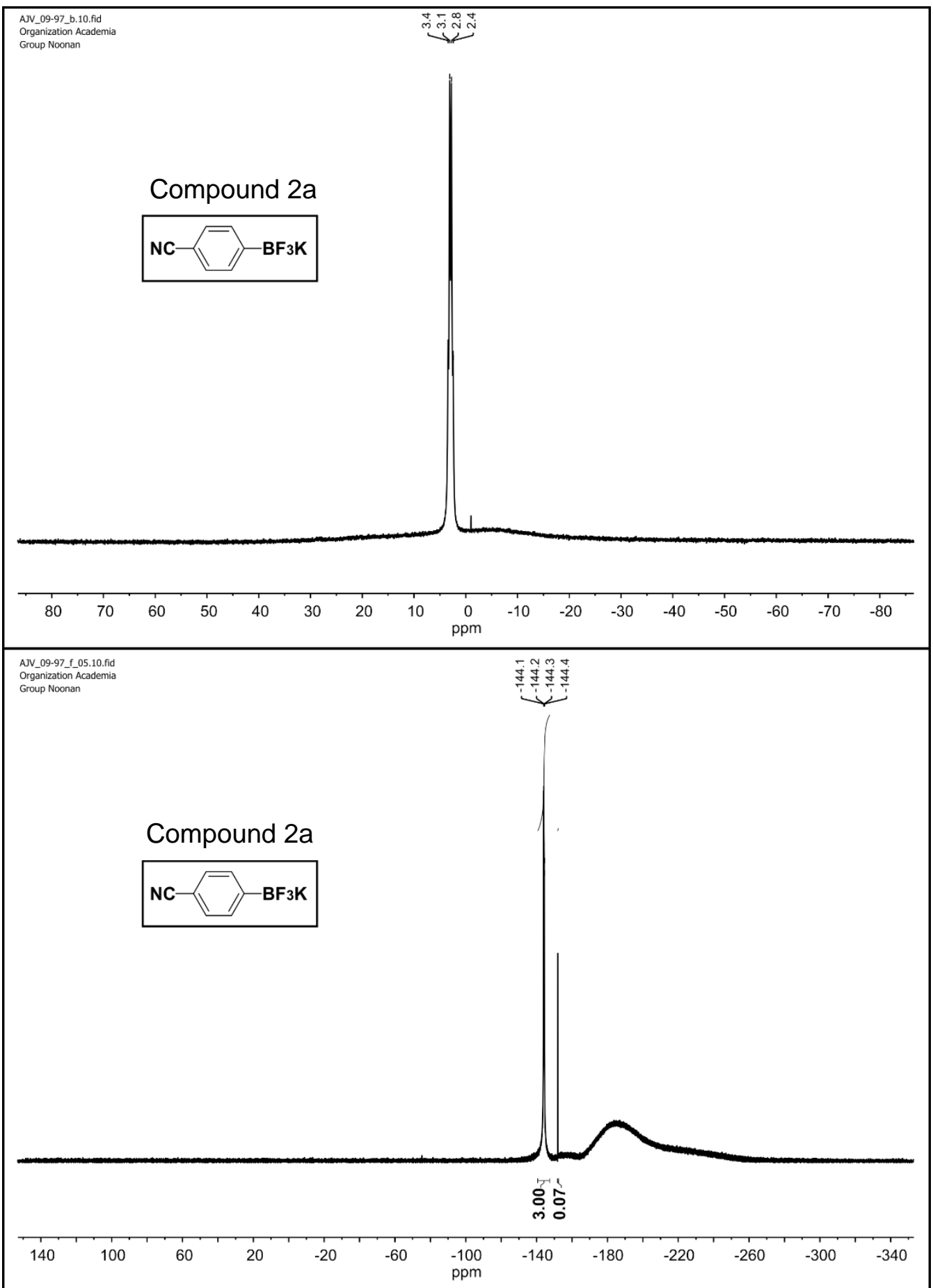

Figure S13b. ${ }^{11} \mathrm{~B}\left\{{ }^{1} \mathrm{H}\right\}$ NMR spectrum (Top, $160 \mathrm{MHz},\left(\mathrm{CD}_{3}\right)_{2} \mathrm{CO}$ ) and ${ }^{19} \mathrm{~F}$ NMR spectrum (Bottom, $470 \mathrm{MHz},\left(\mathrm{CD}_{3}\right)_{2} \mathrm{CO}$ ) of potassium (4-cyanophenyl)trifluoroborate. 


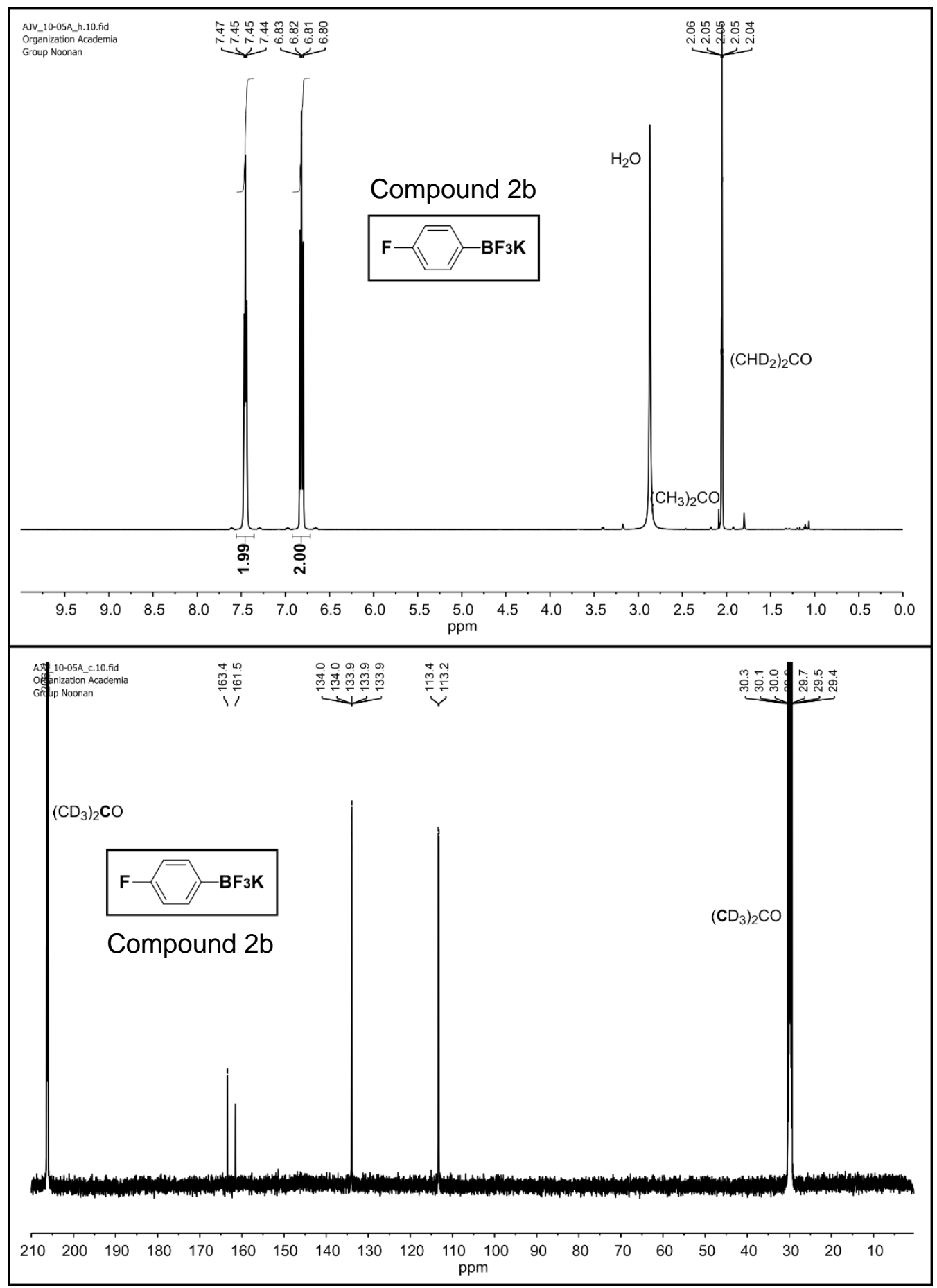

Figure S14a. ${ }^{1} \mathrm{H}$ NMR spectrum (Top, $500 \mathrm{MHz},\left(\mathrm{CD}_{3}\right)_{2} \mathrm{CO}$ ) and ${ }^{13} \mathrm{C}\left\{{ }^{1} \mathrm{H}\right\} \mathrm{NMR}$ spectrum (Bottom, $\left.126 \mathrm{MHz},\left(\mathrm{CD}_{3}\right)_{2} \mathrm{CO}\right)$ of potassium (4-fluorophenyl)trifluoroborate. 


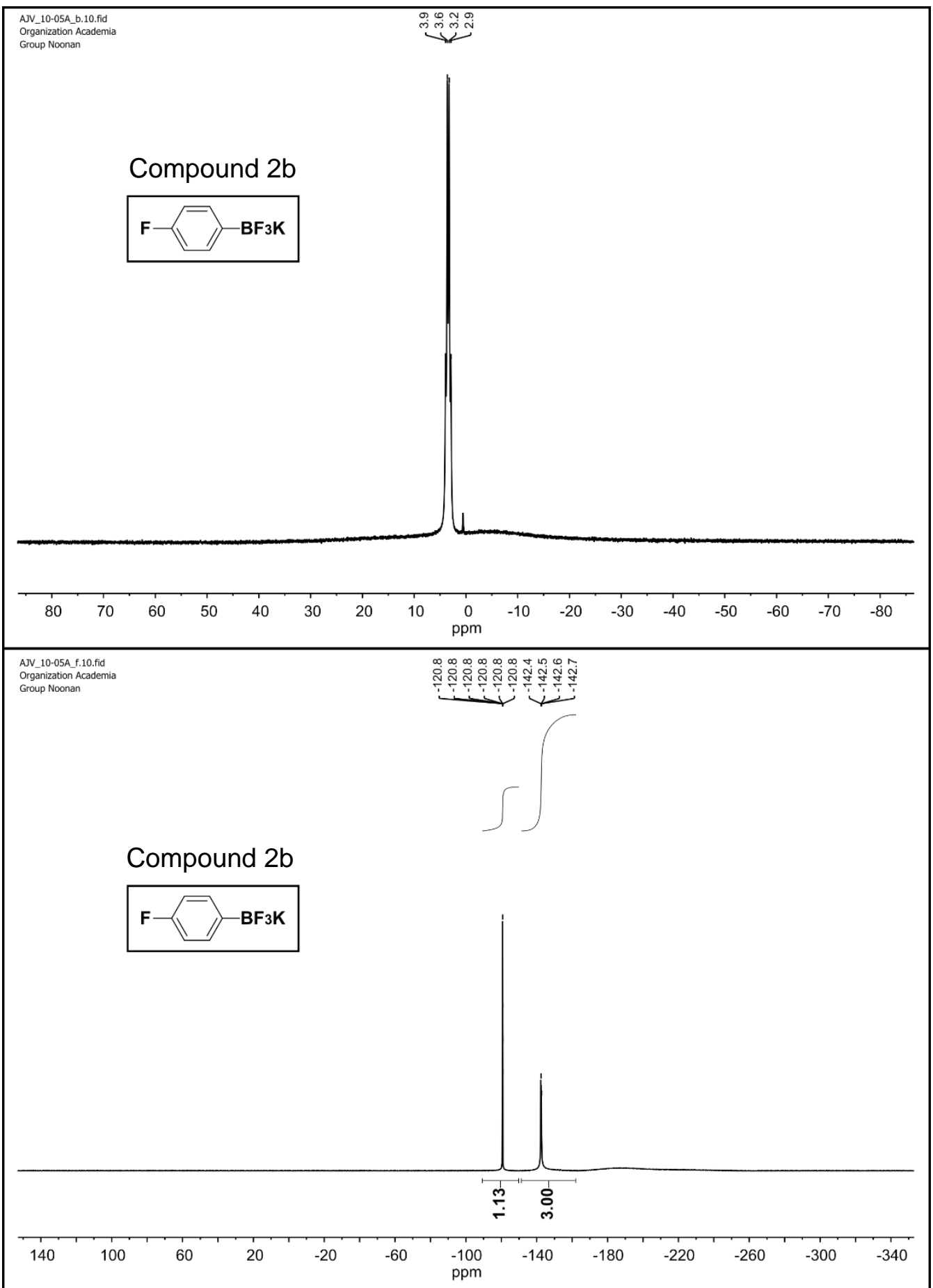

Figure S14b. ${ }^{11} \mathrm{~B}\left\{{ }^{1} \mathrm{H}\right\}$ NMR spectrum (Top, $160 \mathrm{MHz},\left(\mathrm{CD}_{3}\right)_{2} \mathrm{CO}$ ) and ${ }^{19} \mathrm{~F}$ NMR spectrum (Bottom, $\left.470 \mathrm{MHz},\left(\mathrm{CD}_{3}\right)_{2} \mathrm{CO}\right)$ of potassium (4-fluorophenyl)trifluoroborate. 


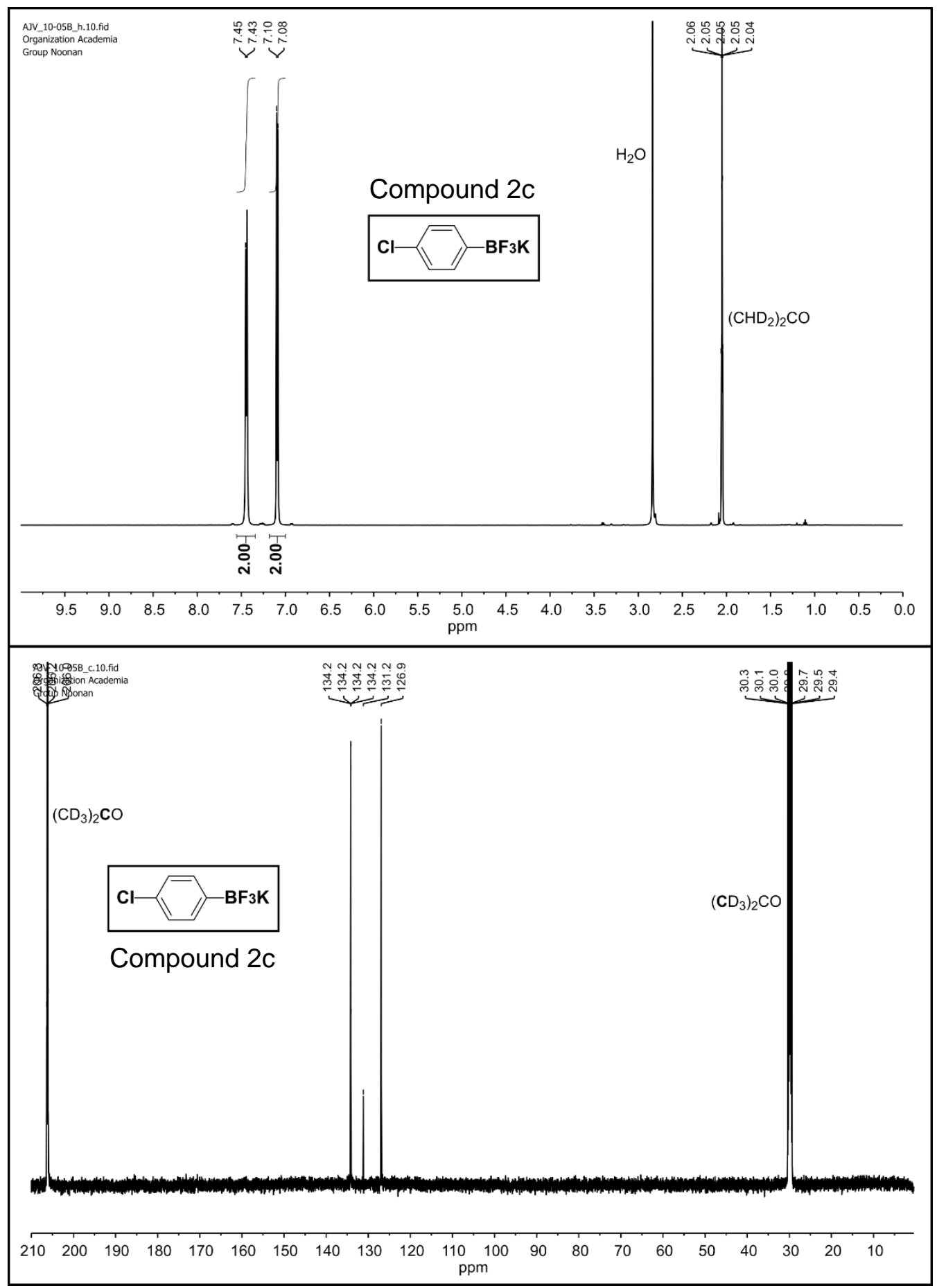

Figure S15a. ${ }^{1} \mathrm{H}$ NMR spectrum (Top, $500 \mathrm{MHz},\left(\mathrm{CD}_{3}\right)_{2} \mathrm{CO}$ ) and ${ }^{13} \mathrm{C}\left\{{ }^{1} \mathrm{H}\right\} \mathrm{NMR}$ spectrum (Bottom, $\left.126 \mathrm{MHz},\left(\mathrm{CD}_{3}\right)_{2} \mathrm{CO}\right)$ of potassium (4-chlorophenyl)trifluoroborate. 


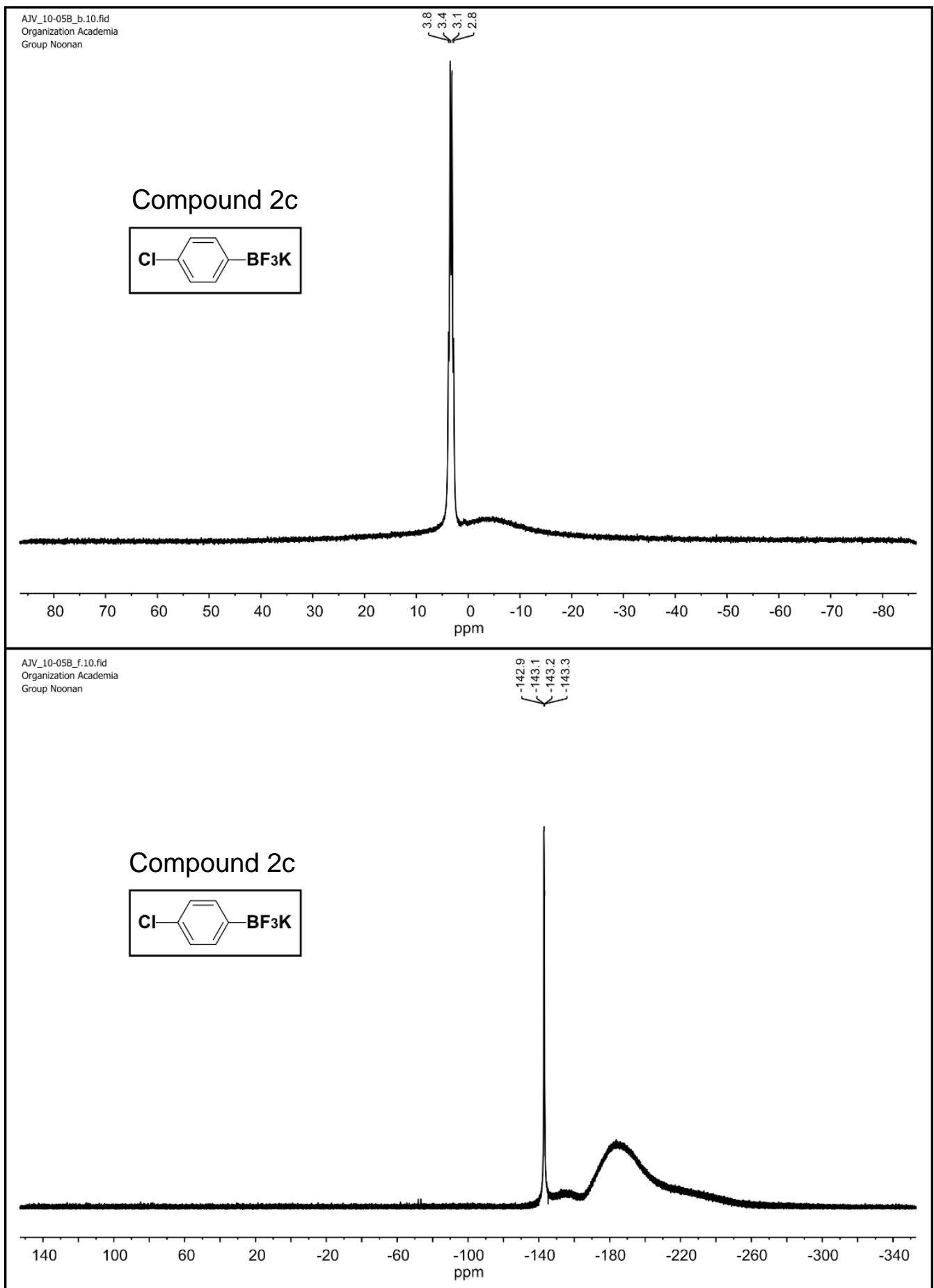

Figure S15b. ${ }^{11} \mathrm{~B}\left\{{ }^{1} \mathrm{H}\right\}$ NMR spectrum (Top, $160 \mathrm{MHz},\left(\mathrm{CD}_{3}\right)_{2} \mathrm{CO}$ ) and ${ }^{19} \mathrm{~F}$ NMR spectrum (Bottom, $470 \mathrm{MHz},\left(\mathrm{CD}_{3}\right)_{2} \mathrm{CO}$ ) of potassium (4-chlorophenyl)trifluoroborate. 


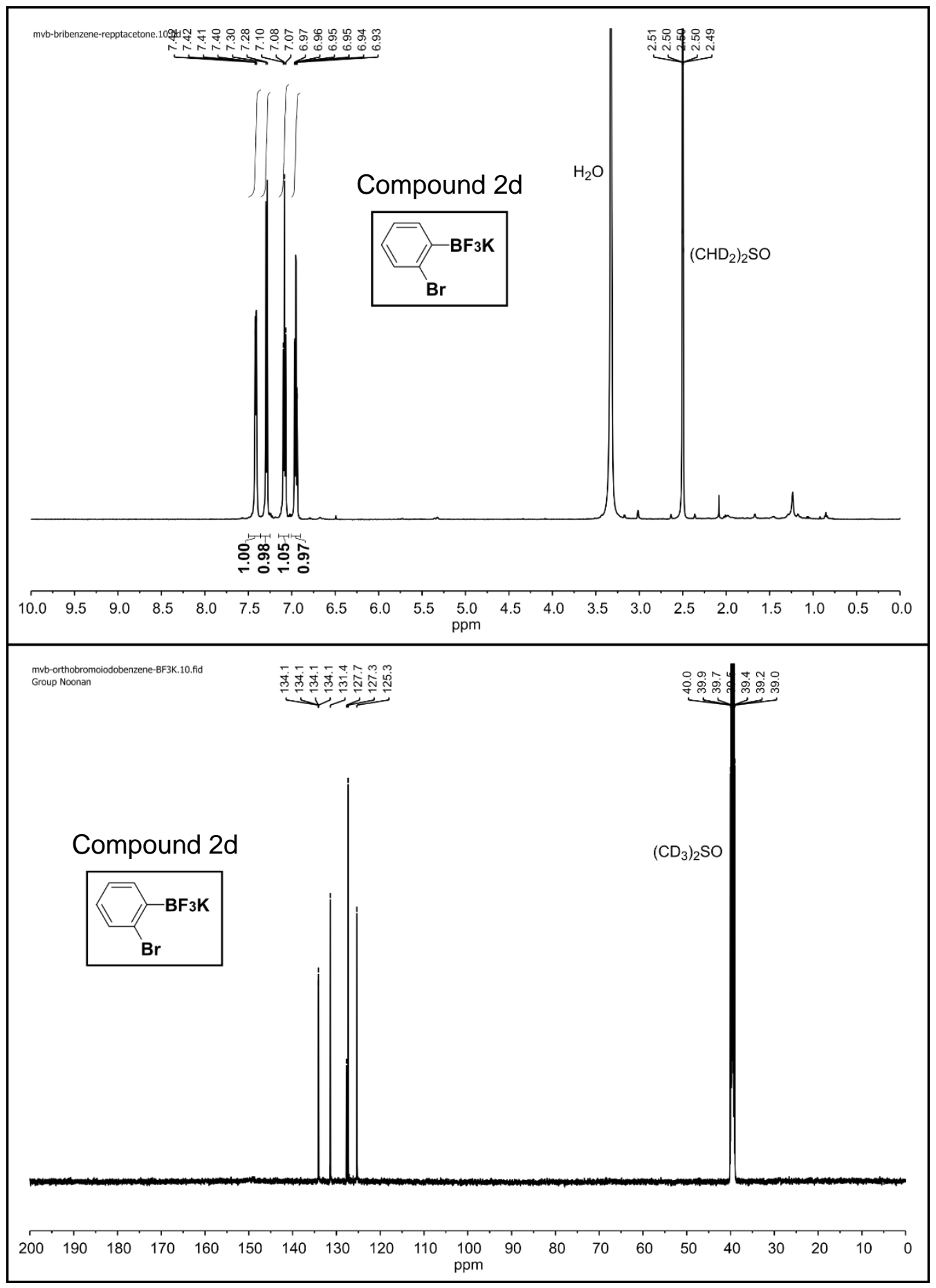

Figure S16a. ${ }^{1} \mathrm{H}$ NMR spectrum (Top, $500 \mathrm{MHz},\left(\mathrm{CD}_{3}\right)_{2} \mathrm{SO}$ ) and ${ }^{13} \mathrm{C}\left\{{ }^{1} \mathrm{H}\right\} \mathrm{NMR}$ spectrum (Bottom, $126 \mathrm{MHz},\left(\mathrm{CD}_{3}\right)_{2} \mathrm{SO}$ ) of potassium (2-bromophenyl)trifluoroborate. 


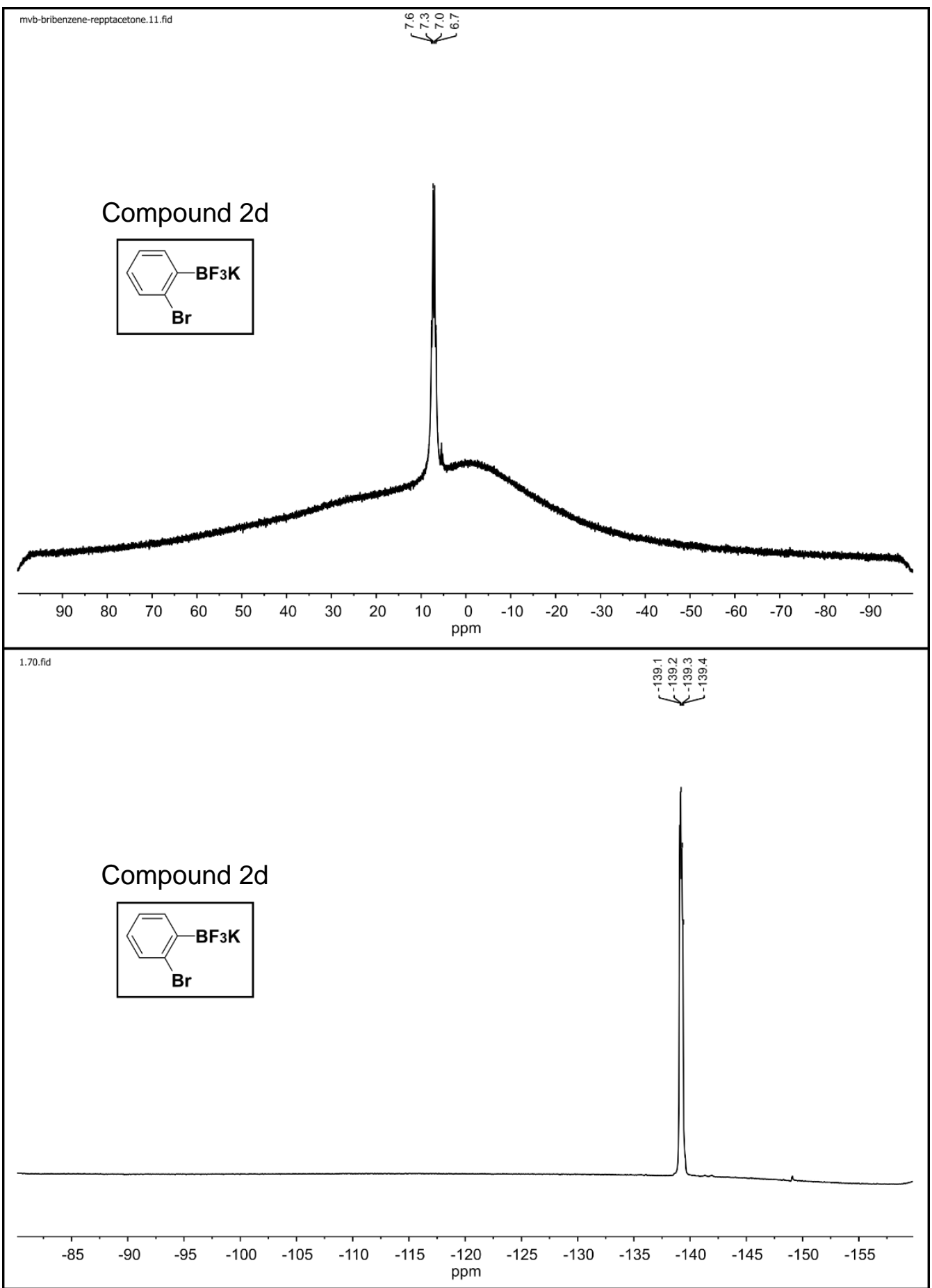

Figure S16b. ${ }^{11} \mathrm{~B}\left\{{ }^{1} \mathrm{H}\right\}$ NMR spectrum (Top, $160 \mathrm{MHz},\left(\mathrm{CD}_{3}\right)_{2} \mathrm{SO}$ ) and ${ }^{19} \mathrm{~F}$ NMR spectrum (Bottom, $470 \mathrm{MHz},\left(\mathrm{CD}_{3}\right)_{2} \mathrm{SO}$ ) of potassium (2-bromophenyl)trifluoroborate. 


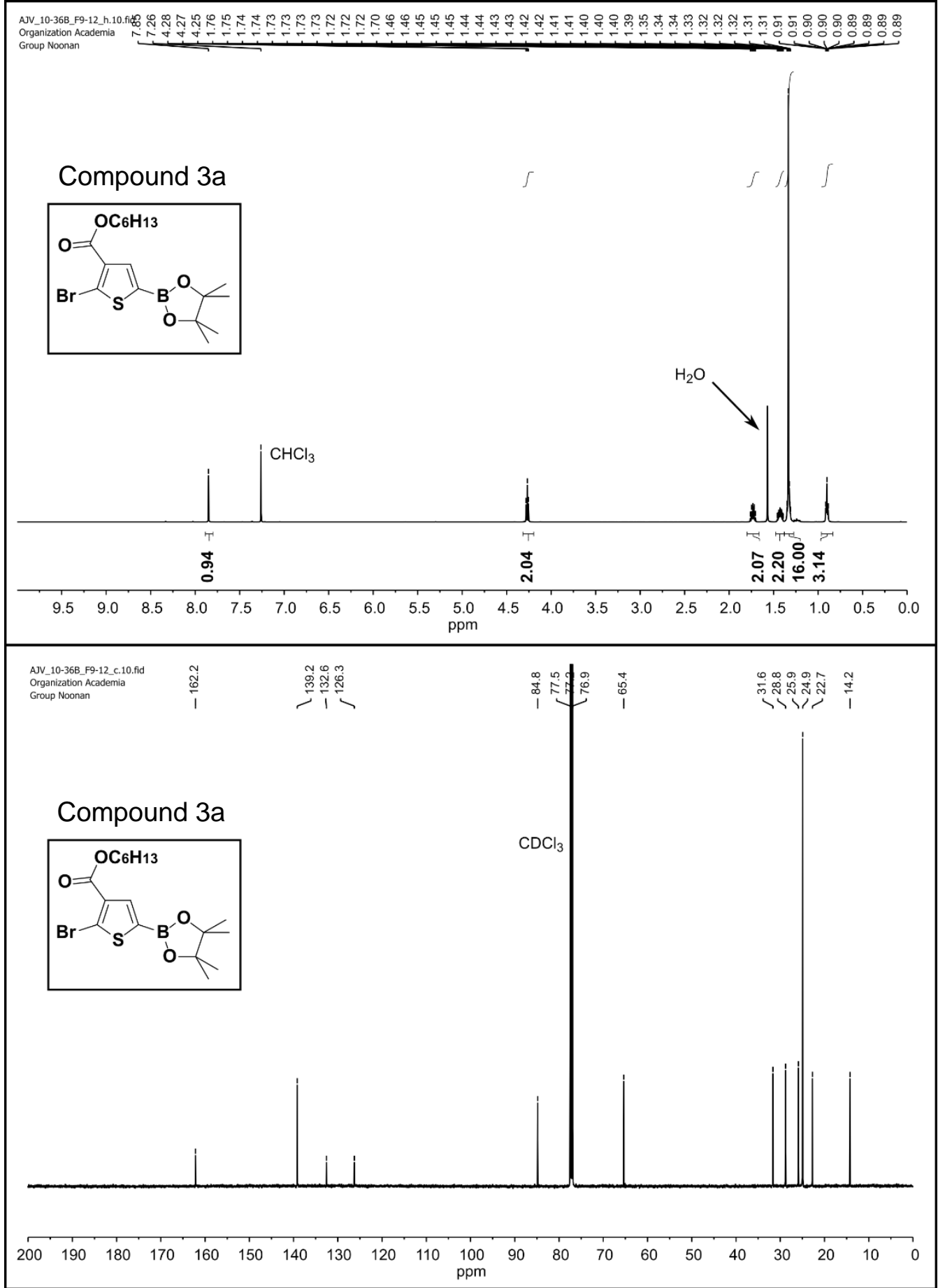

Figure S17a. ${ }^{1} \mathrm{H}$ NMR spectrum (Top, $500 \mathrm{MHz}, \mathrm{CDCl}_{3}$ ) and ${ }^{13} \mathrm{C}\left\{{ }^{1} \mathrm{H}\right\} \mathrm{NMR}$ spectrum (Bottom, $\left.126 \mathrm{MHz}, \mathrm{CDCl}_{3}\right)$ of hexyl 2-bromo-5-(4,4,5,5-tetramethyl-1,3,2-dioxaborolan-2yl)thiophene-3-carboxylate. 


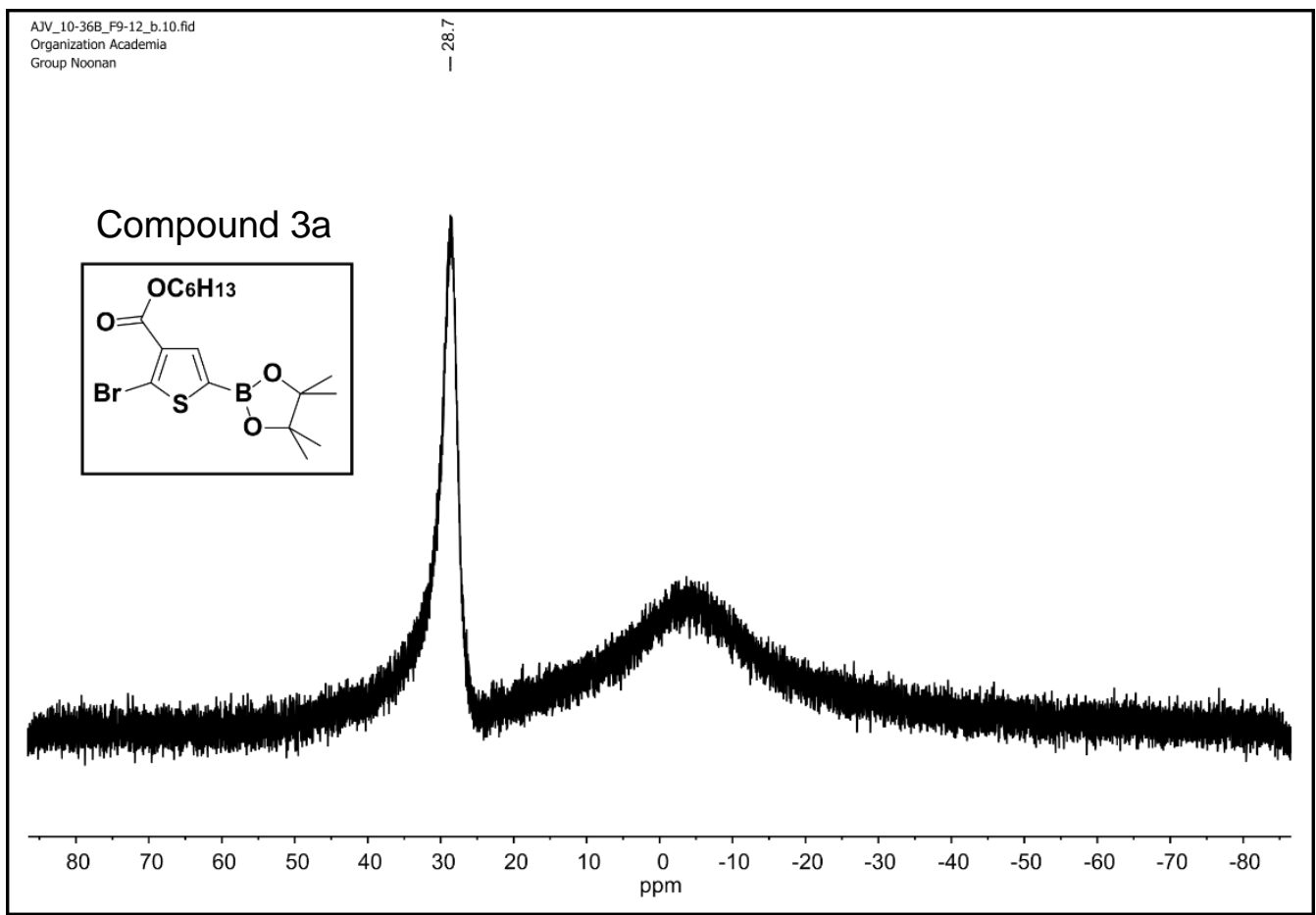

Figure S17b. ${ }^{11} \mathrm{~B}\left\{{ }^{1} \mathrm{H}\right\}$ NMR spectrum $\left(160 \mathrm{MHz}, \mathrm{CDCl}_{3}\right)$ of hexyl 2-bromo-5-(4,4,5,5tetramethyl-1,3,2-dioxaborolan-2-yl)thiophene-3-carboxylate. 


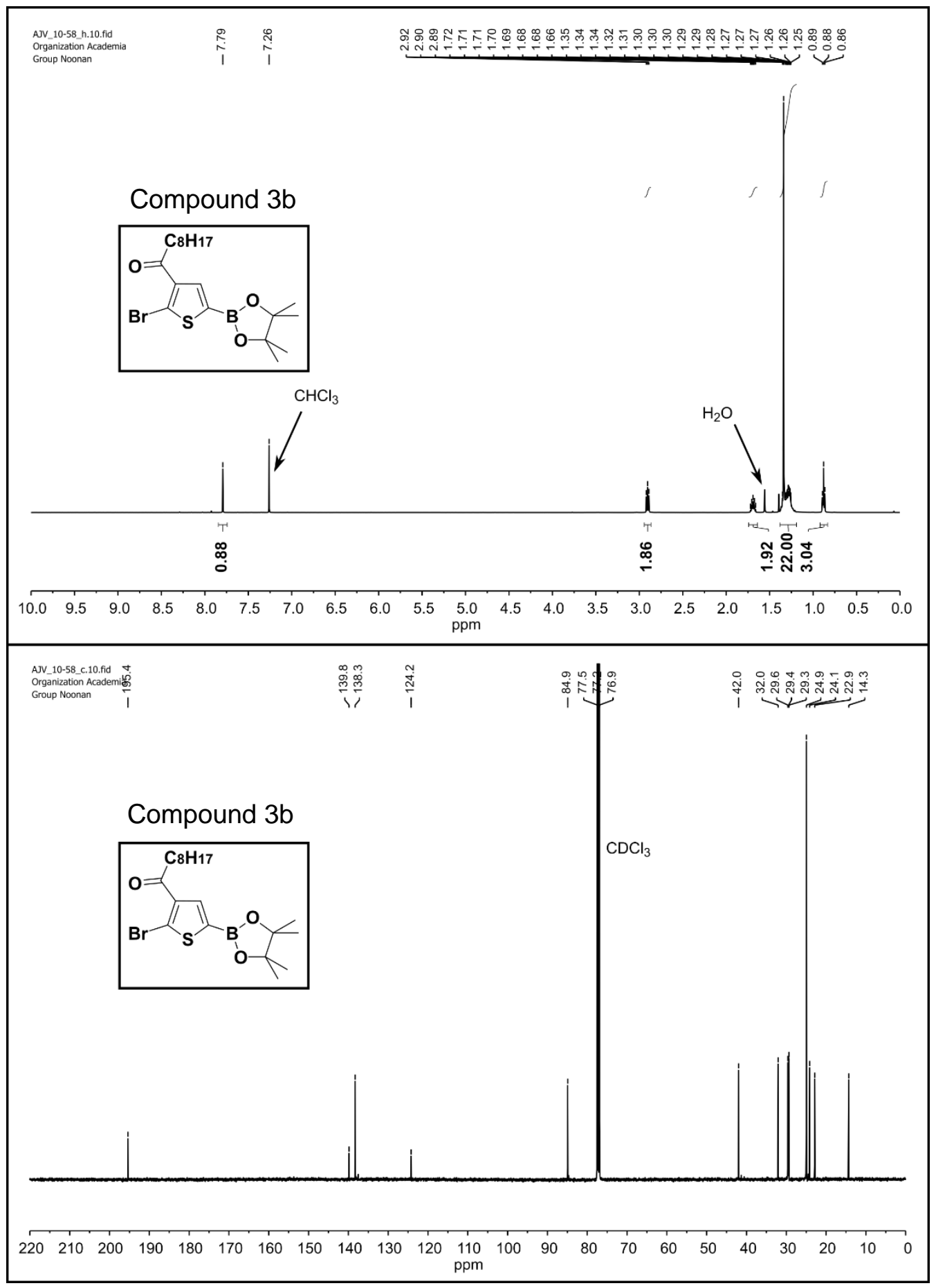

Figure S18a. ${ }^{1} \mathrm{H}$ NMR spectrum (Top, $500 \mathrm{MHz}, \mathrm{CDCl}_{3}$ ) and ${ }^{13} \mathrm{C}\left\{{ }^{1} \mathrm{H}\right\} \mathrm{NMR}$ spectrum (Bottom, $\left.126 \mathrm{MHz}, \mathrm{CDCl}_{3}\right)$ of 1-(2-bromo-5-(4,4,5,5-tetramethyl-1,3,2-dioxaborolan-2-yl)thiophen-3yl)nonan-1-one. 


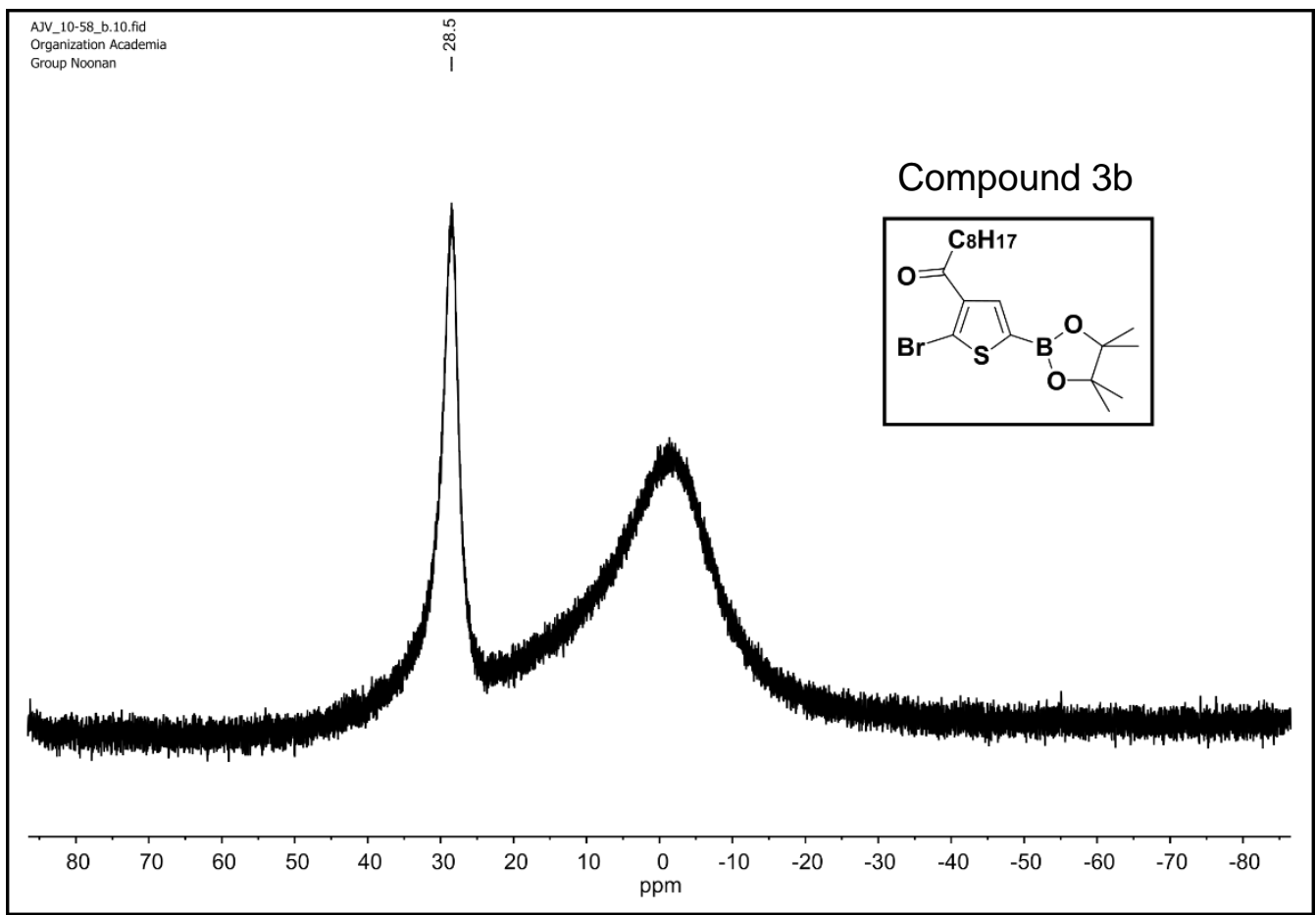

Figure S18b. ${ }^{11} \mathrm{~B}\left\{{ }^{1} \mathrm{H}\right\}$ NMR spectrum $\left(160 \mathrm{MHz}, \mathrm{CDCl}_{3}\right)$ of 1-(2-bromo-5-(4,4,5,5-tetramethyl1,3,2-dioxaborolan-2-yl)thiophen-3-yl)nonan-1-one. 


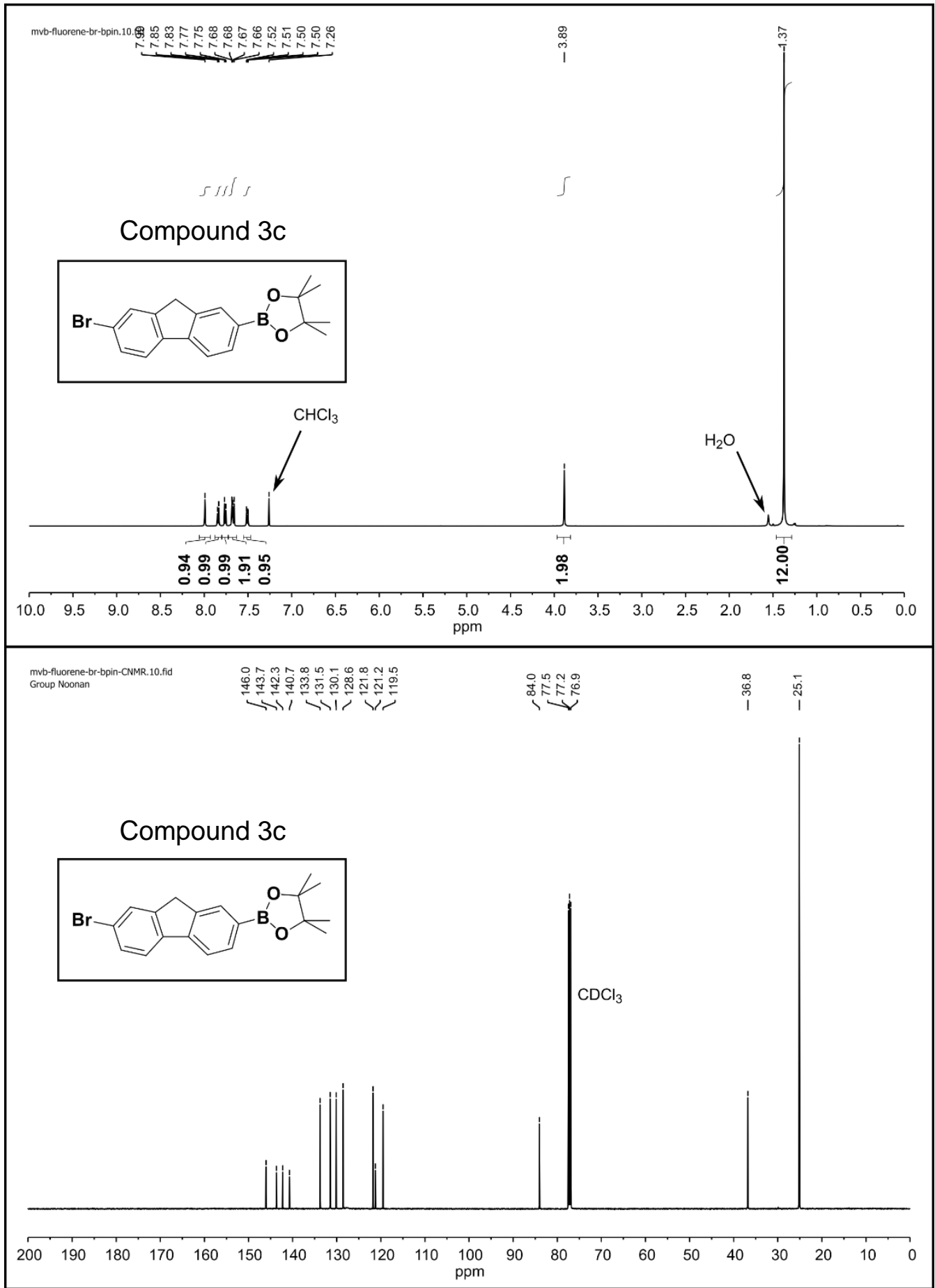

Figure S19a. ${ }^{1} \mathrm{H}$ NMR spectrum (Top, $500 \mathrm{MHz}, \mathrm{CDCl}_{3}$ ) and ${ }^{13} \mathrm{C}\left\{{ }^{1} \mathrm{H}\right\} \mathrm{NMR}$ spectrum (Bottom, $126 \mathrm{MHz}_{\mathrm{CDCl}}$ ) of 2-(7-bromo-9H-fluoren-2-yl)-4,4,5,5-tetramethyl-1,3,2-dioxaborolane. 


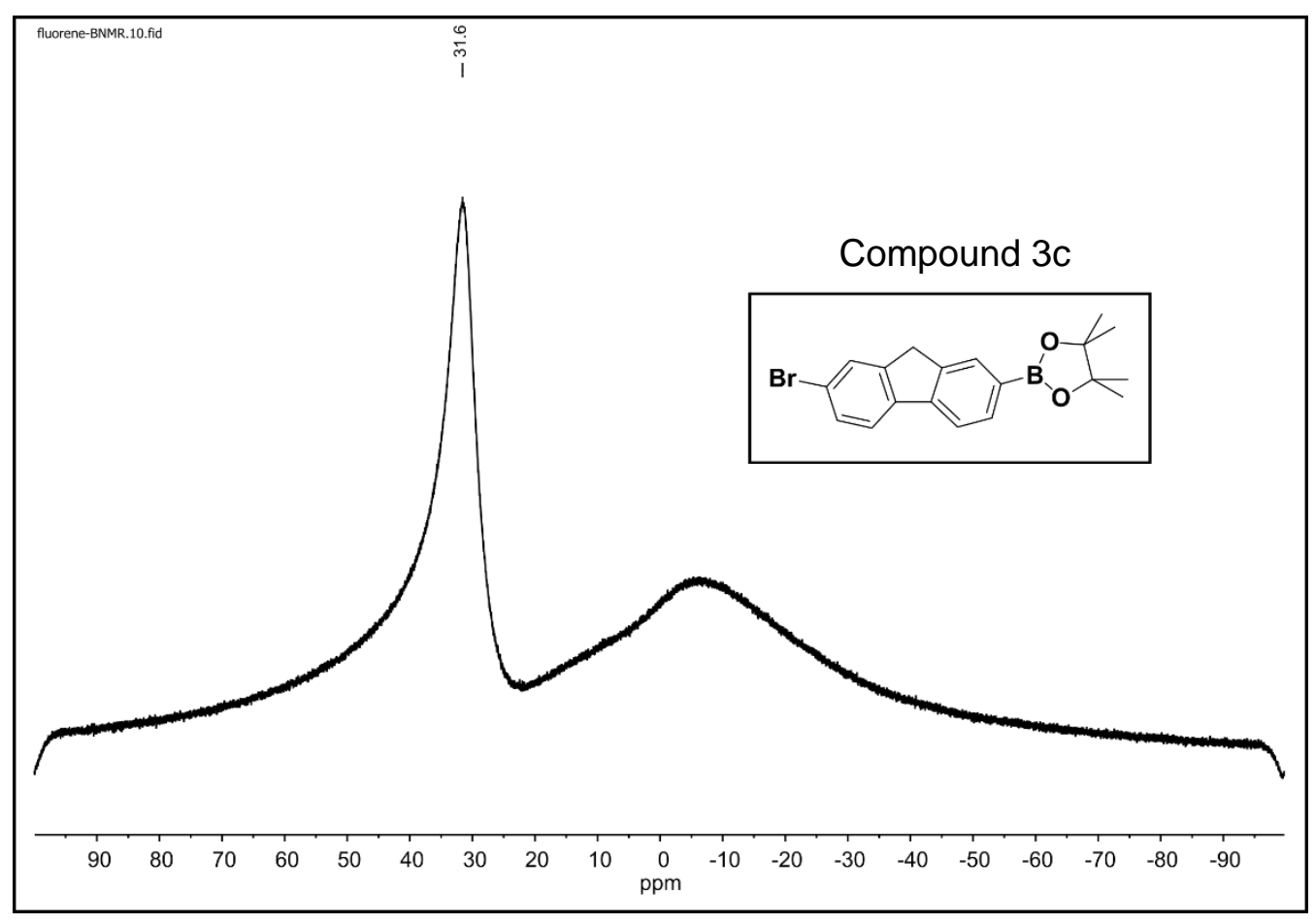

Figure S19b. ${ }^{11} \mathrm{~B}\left\{{ }^{1} \mathrm{H}\right\}$ NMR spectrum (160 MHz, $\left.\mathrm{CDCl}_{3}\right)$ of 2-(7-bromo-9H-fluoren-2-yl)4,4,5,5-tetramethyl-1,3,2-dioxaborolane. 


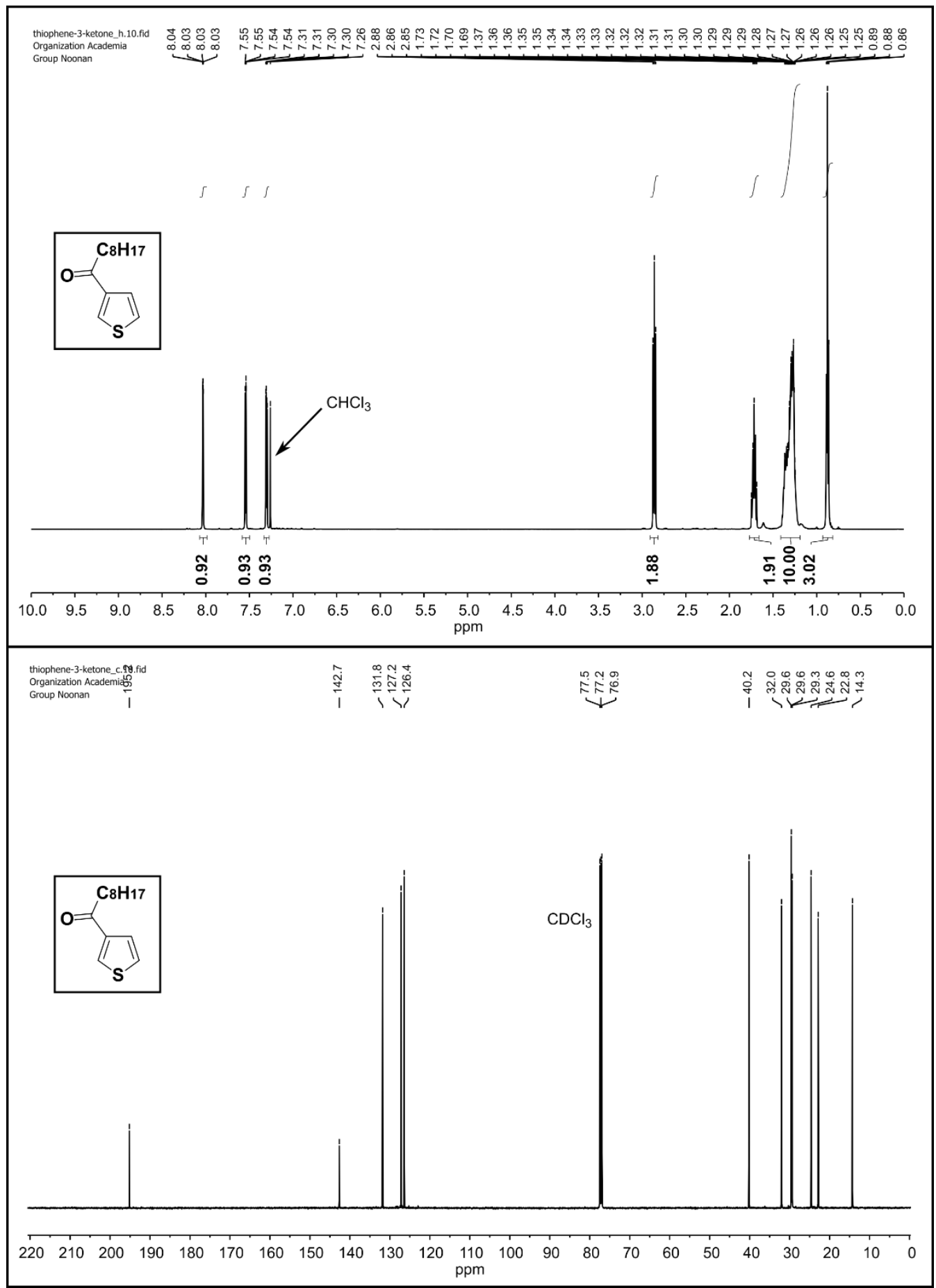

Figure S20. ${ }^{1} \mathrm{H}$ NMR spectrum (Top, $500 \mathrm{MHz}, \mathrm{CDCl}_{3}$ ) and ${ }^{13} \mathrm{C}\left\{{ }^{1} \mathrm{H}\right\}$ NMR spectrum (Bottom, $\left.126 \mathrm{MHz}, \mathrm{CDCl}_{3}\right)$ of 1-(thiophen-3-yl)nonan-1-one. 


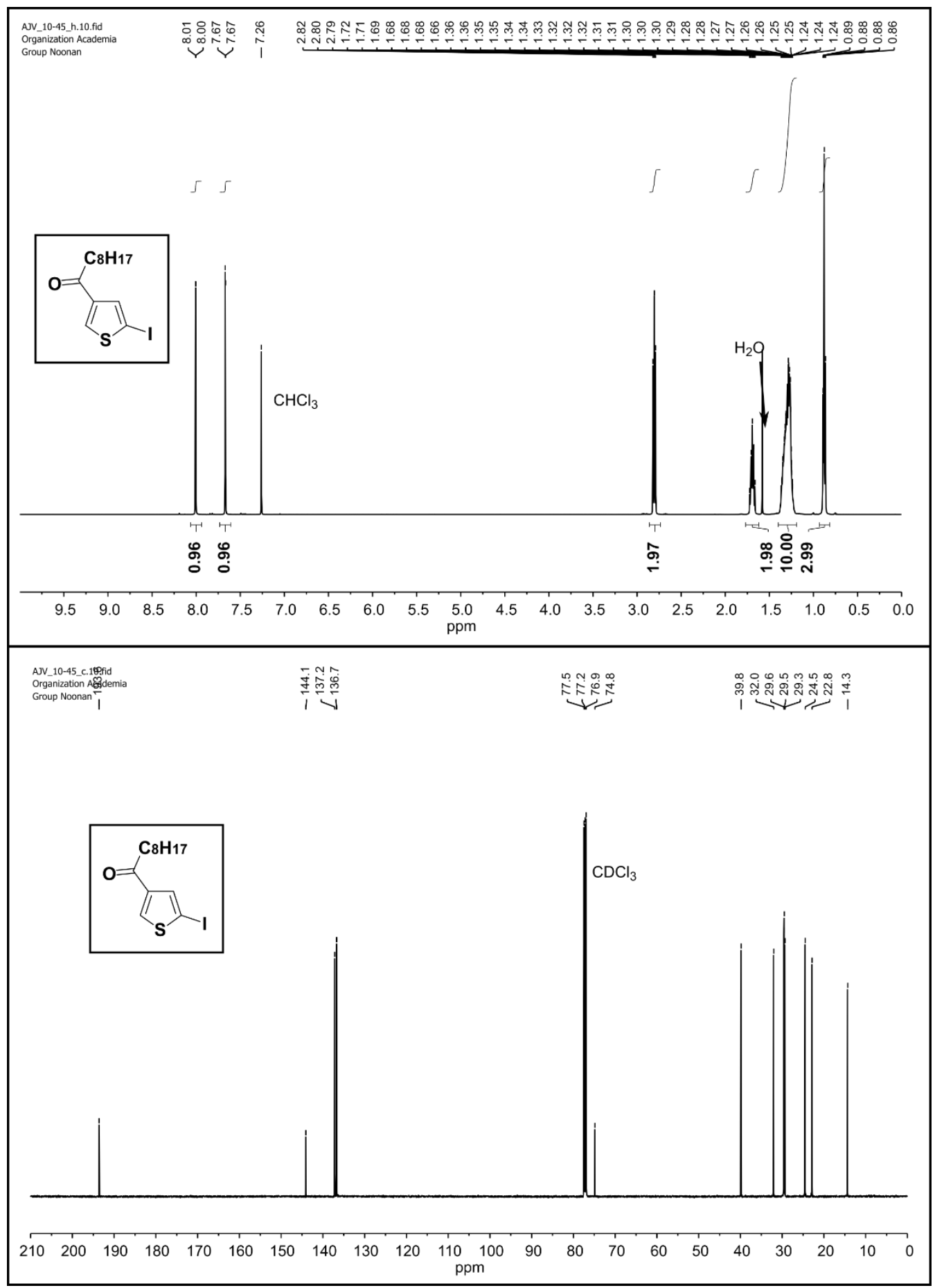

Figure S21. ${ }^{1} \mathrm{H}$ NMR spectrum (Top, $500 \mathrm{MHz}, \mathrm{CDCl}_{3}$ ) and ${ }^{13} \mathrm{C}\left\{{ }^{1} \mathrm{H}\right\} \mathrm{NMR}$ spectrum (Bottom, $\left.126 \mathrm{MHz}, \mathrm{CDCl}_{3}\right)$ of 1-(5-iodothiophen-3-yl)nonan-1-one. 


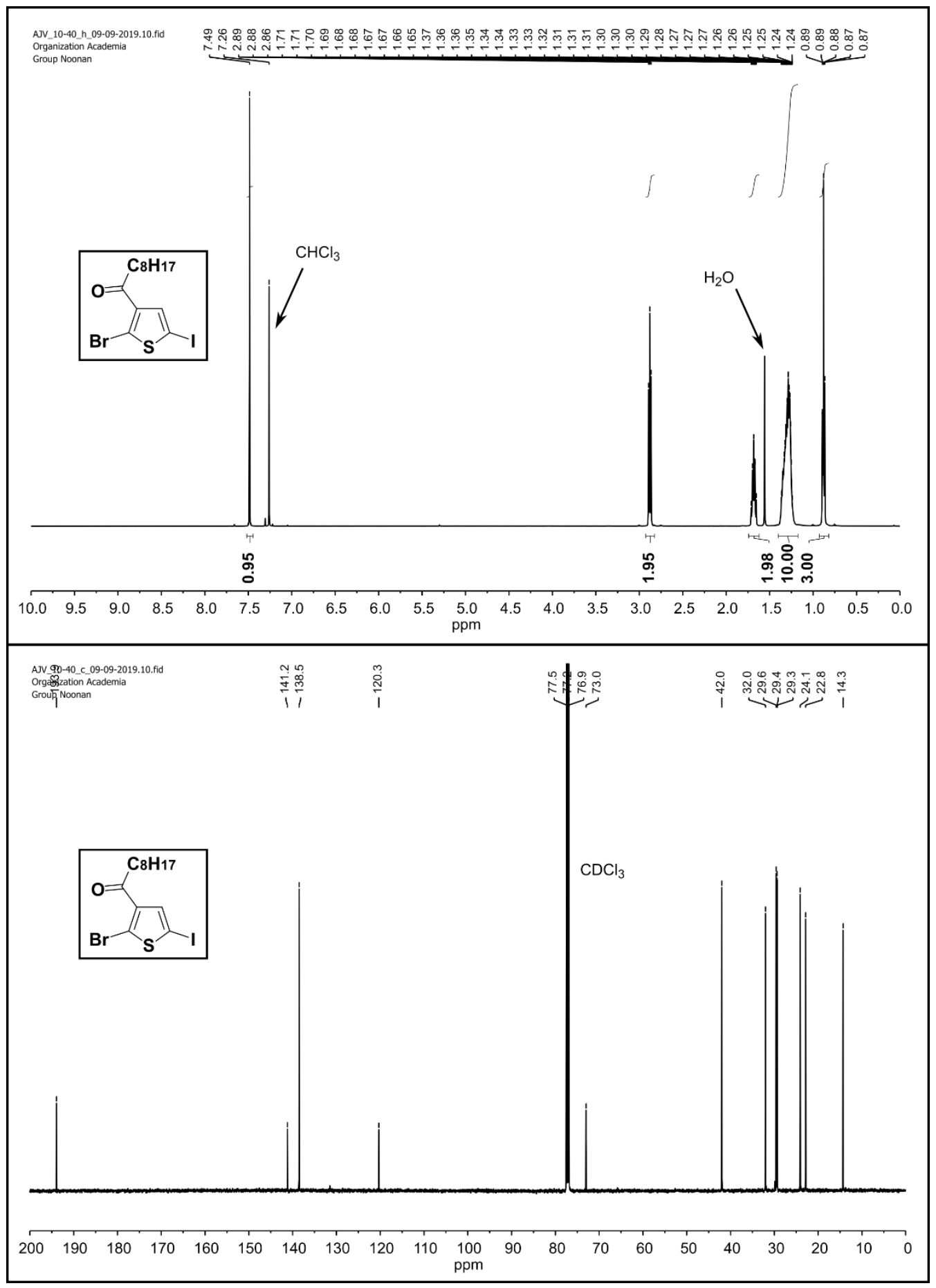

Figure S22. ${ }^{1} \mathrm{H}$ NMR spectrum (Top, $500 \mathrm{MHz}, \mathrm{CDCl}_{3}$ ) and ${ }^{13} \mathrm{C}\left\{{ }^{1} \mathrm{H}\right\} \mathrm{NMR}$ spectrum (Bottom, $\left.126 \mathrm{MHz}, \mathrm{CDCl}_{3}\right)$ of 1-(2-bromo-5-iodothiophen-3-yl)nonan-1-one. 


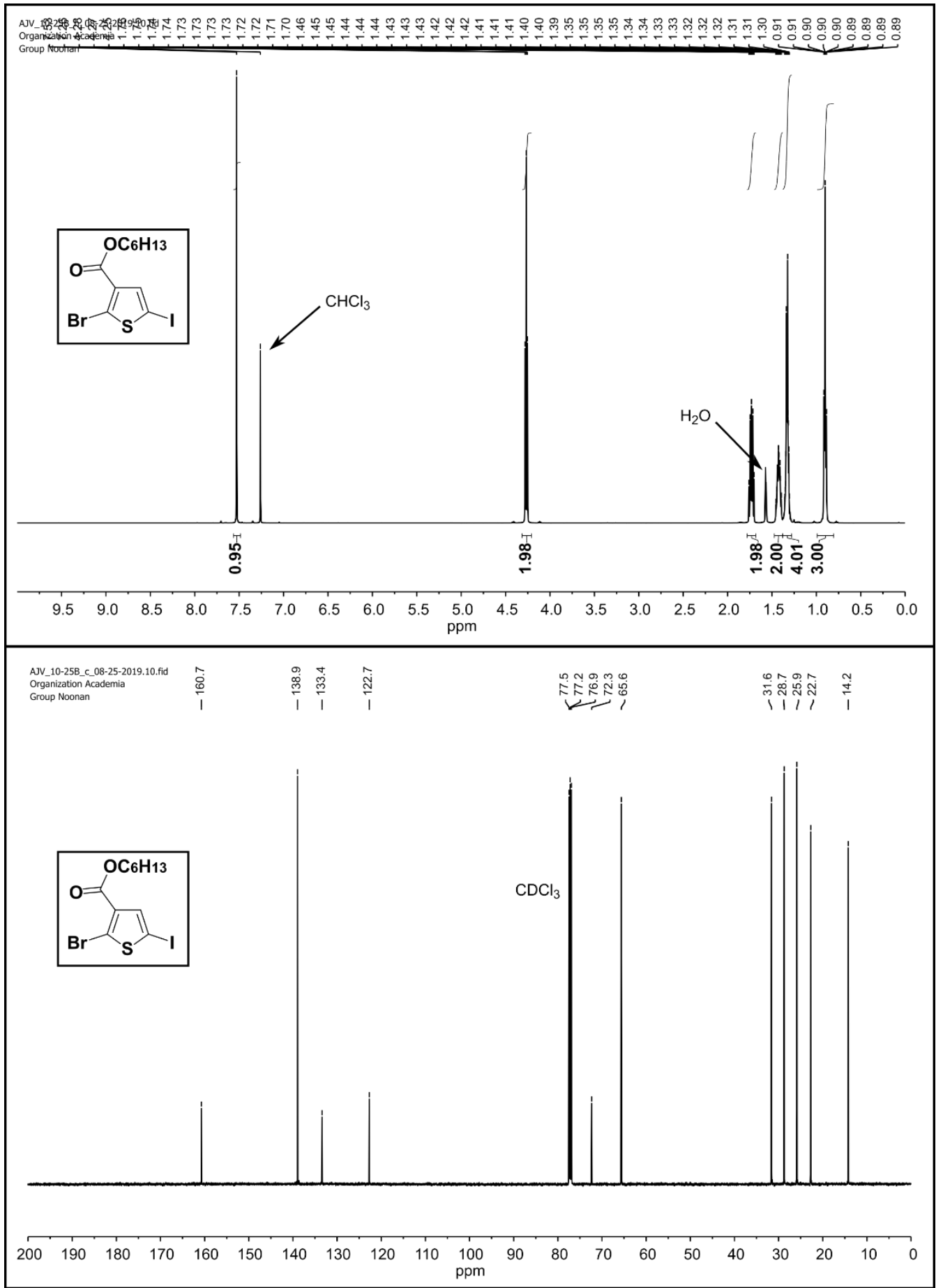

Figure S23. ${ }^{1} \mathrm{H}$ NMR spectrum (Top, $500 \mathrm{MHz}, \mathrm{CDCl}_{3}$ ) and ${ }^{13} \mathrm{C}\left\{{ }^{1} \mathrm{H}\right\}$ NMR spectrum (Bottom, $126 \mathrm{MHz}, \mathrm{CDCl}_{3}$ ) of hexyl 2-bromo-5-iodothiophene-3-carboxylate. 


\section{HRMS}

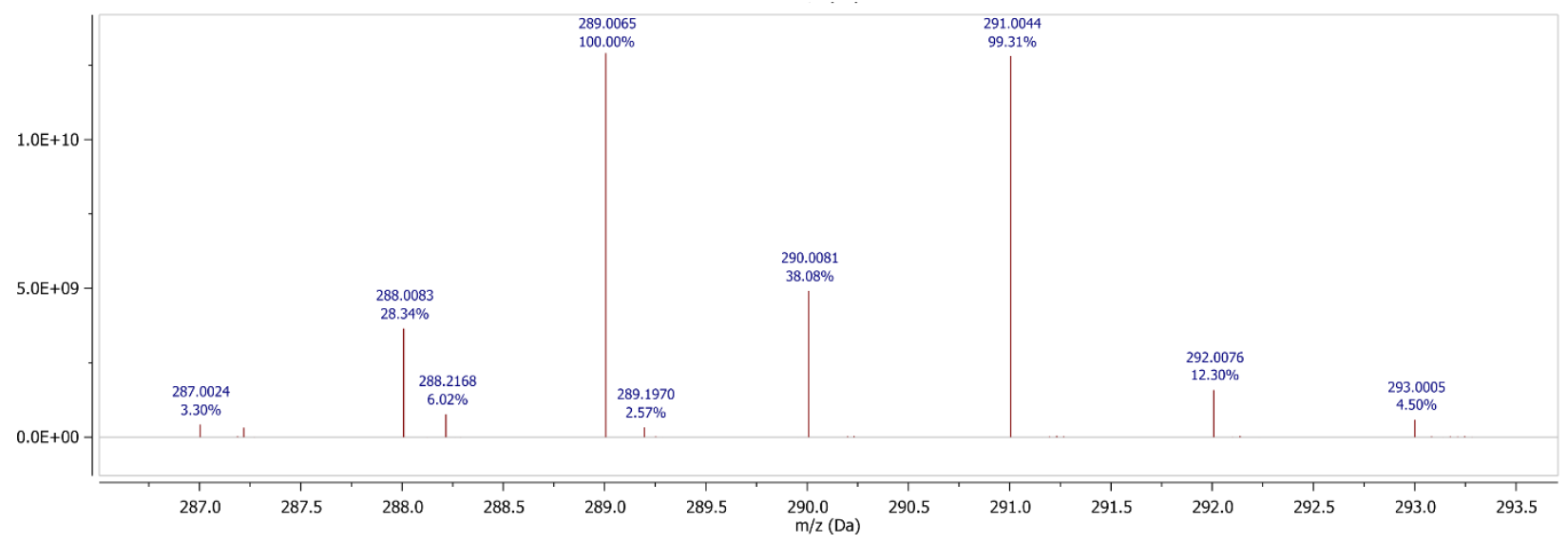

Figure S24. DART-MS of 2-(5-bromothiophen-2-yl)-4,4,5,5-tetramethyl-1,3,2-dioxaborolane (Compound 1a).

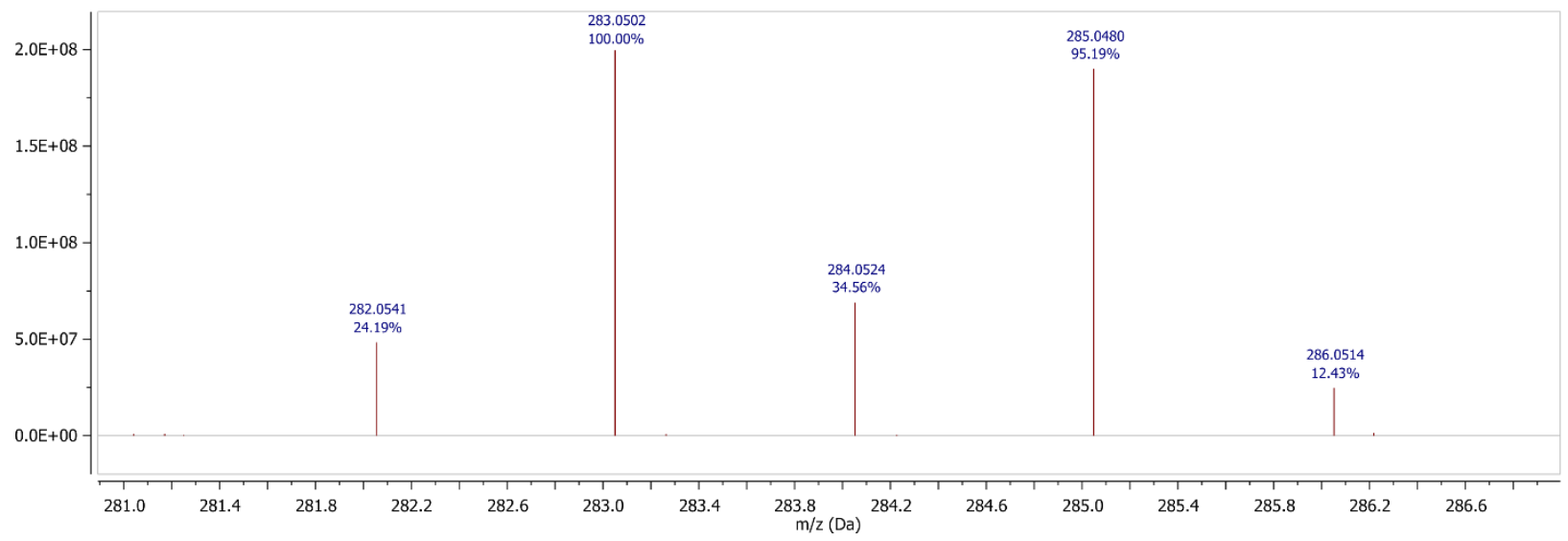

Figure S25. DART-MS of 2-(4-bromophenyl)-4,4,5,5-tetramethyl-1,3,2-dioxaborolane (Compound 1b). 


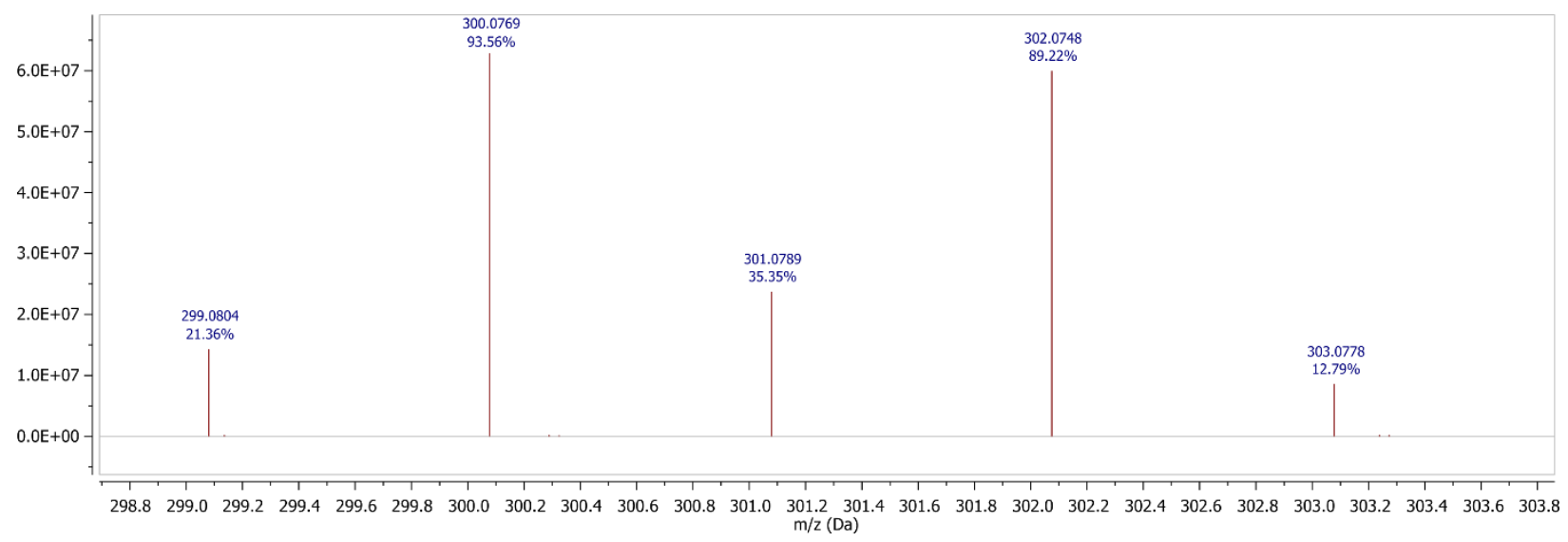

Figure S26. DART-MS of 2-(3-bromophenyl)-4,4,5,5-tetramethyl-1,3,2-dioxaborolane (Compound 1c).

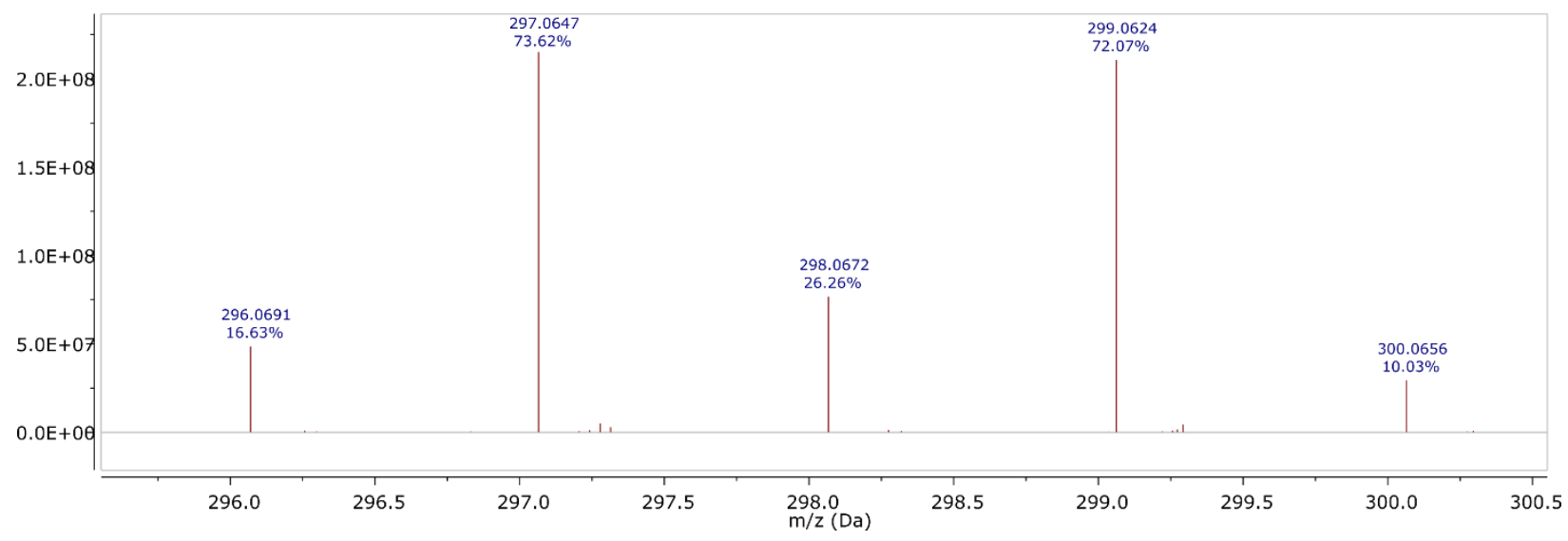

Figure S27. DART-MS of 2-(4-bromo-3-methylphenyl)-4,4,5,5-tetramethyl-1,3,2dioxaborolane (Compound 1d). 


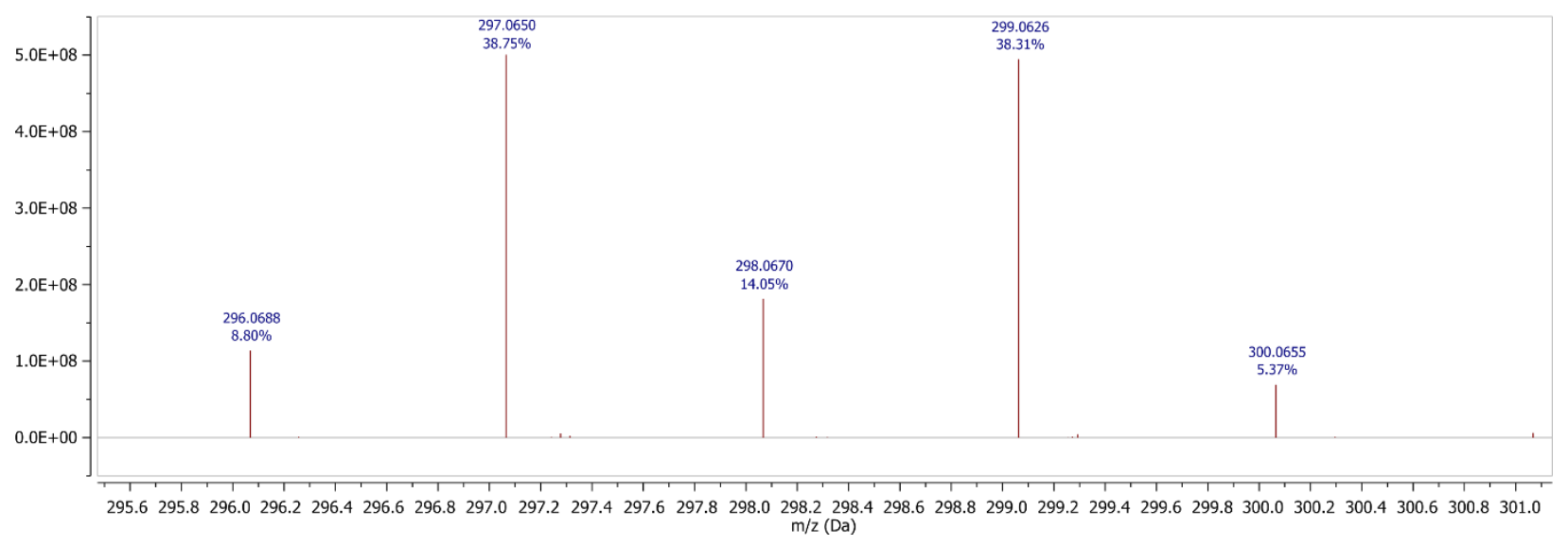

Figure S28. DART-MS of 2-(4-bromo-2-methylphenyl)-4,4,5,5-tetramethyl-1,3,2dioxaborolane (Compound $1 \mathrm{e})$.

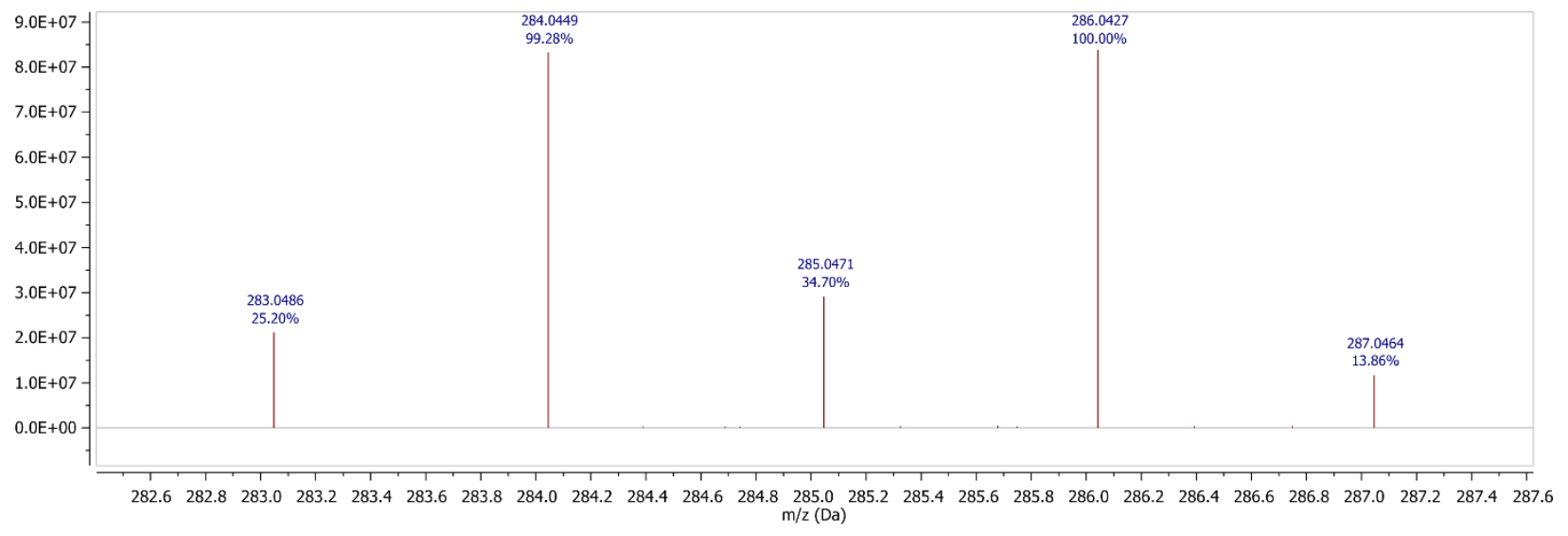

Figure S29. DART-MS of 2-bromo-5-(4,4,5,5-tetramethyl-1,3,2-dioxaborolan-2-yl)pyridine (Compound 1f). 


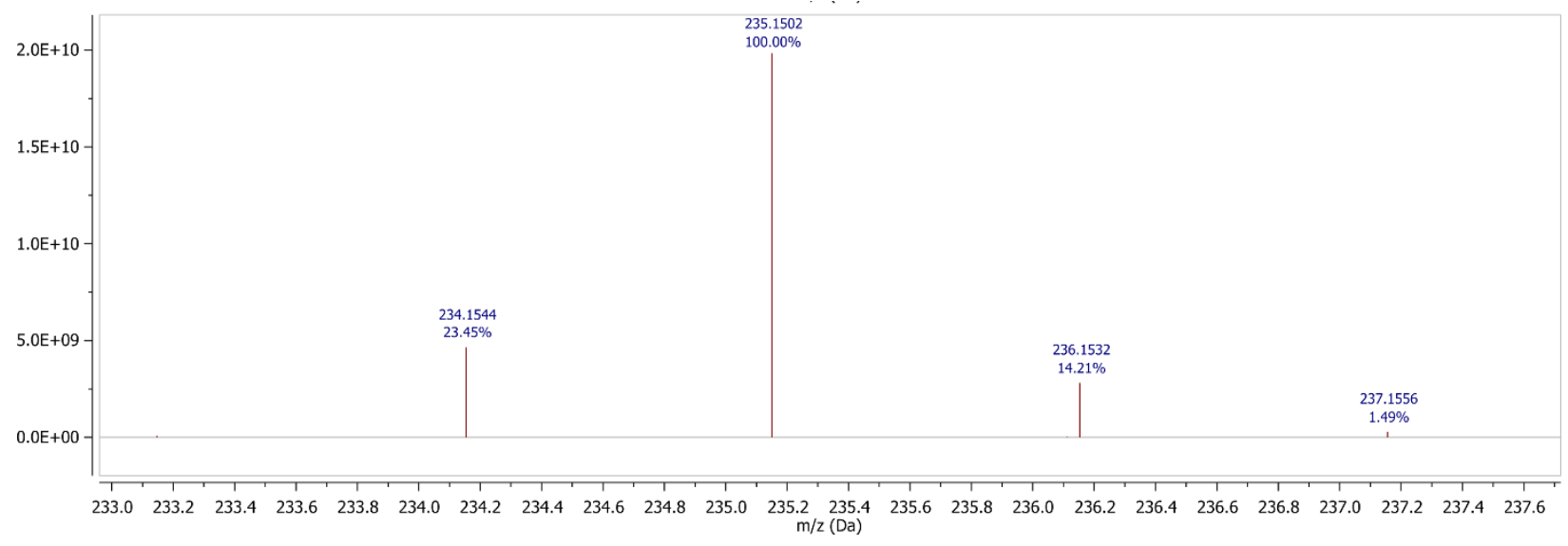

Figure S30. DART-MS of 2-(4-methoxyphenyl)-4,4,5,5-tetramethyl-1,3,2-dioxaborolane (Compound 1g).

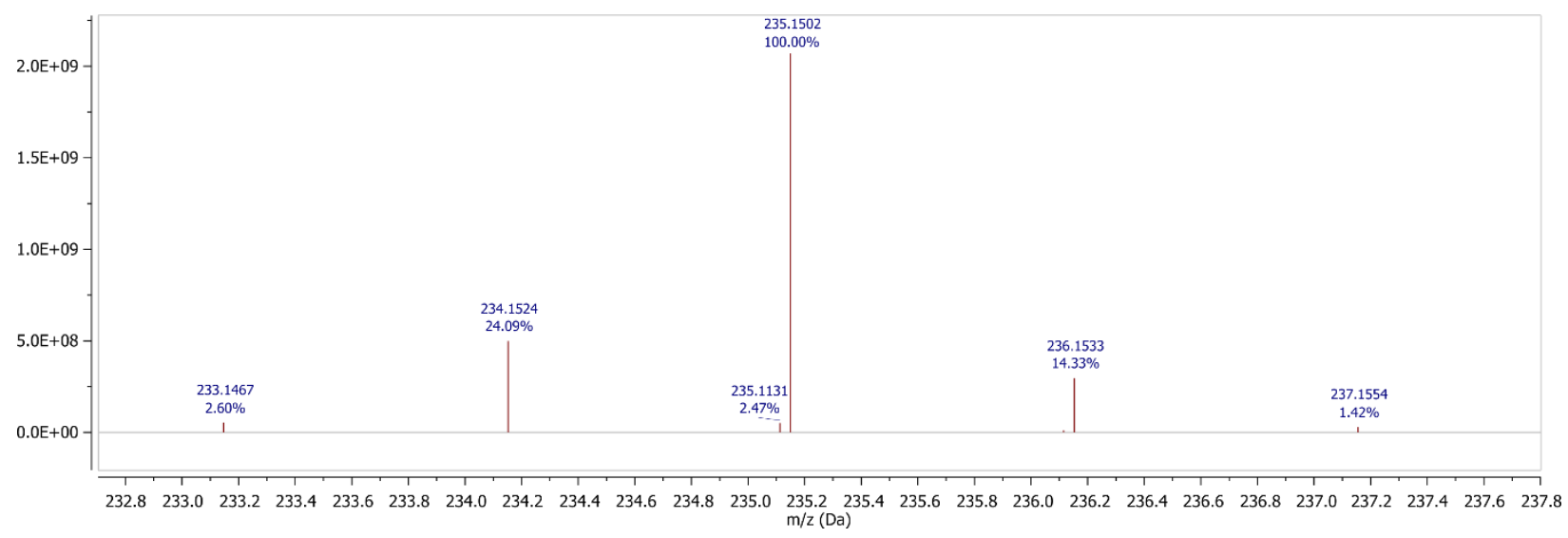

Figure S31. DART-MS of 2-(2-methoxyphenyl)-4,4,5,5-tetramethyl-1,3,2-dioxaborolane (Compound 1h). 


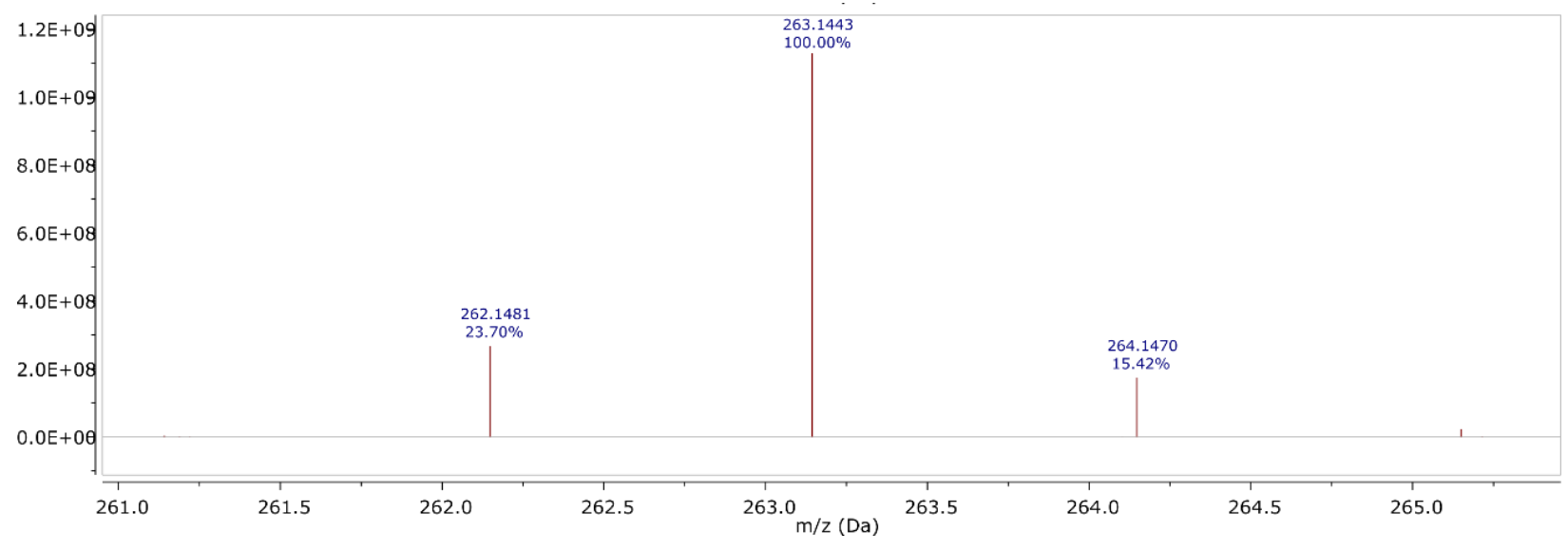

Figure S32. DART-MS of methyl 4-(4,4,5,5-tetramethyl-1,3,2-dioxaborolan-2-yl)benzoate (Compound 1i).

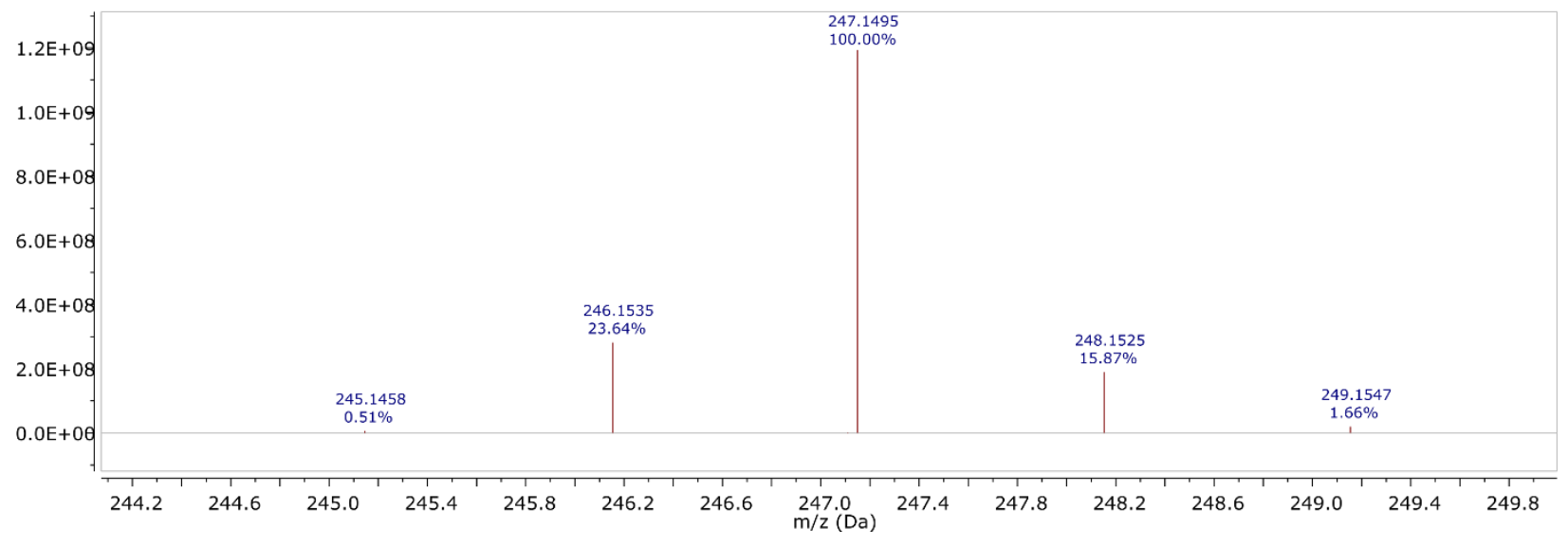

Figure S33. DART-MS of 1-(4-(4,4,5,5-tetramethyl-1,3,2-dioxaborolan-2-yl)phenyl)ethan-1one (Compound $1 \mathrm{j}$ ). 


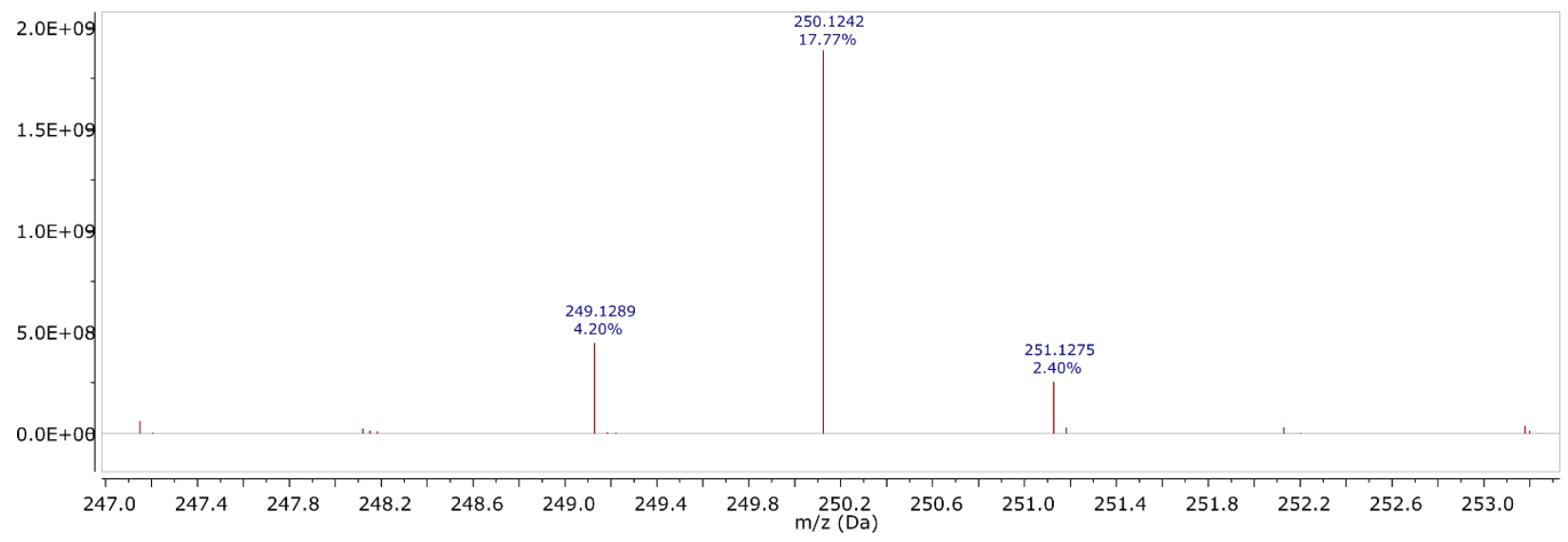

Figure S34. DART-MS of 4,4,5,5-tetramethyl-2-(4-nitrophenyl)-1,3,2-dioxaborolane (Compound 1k).

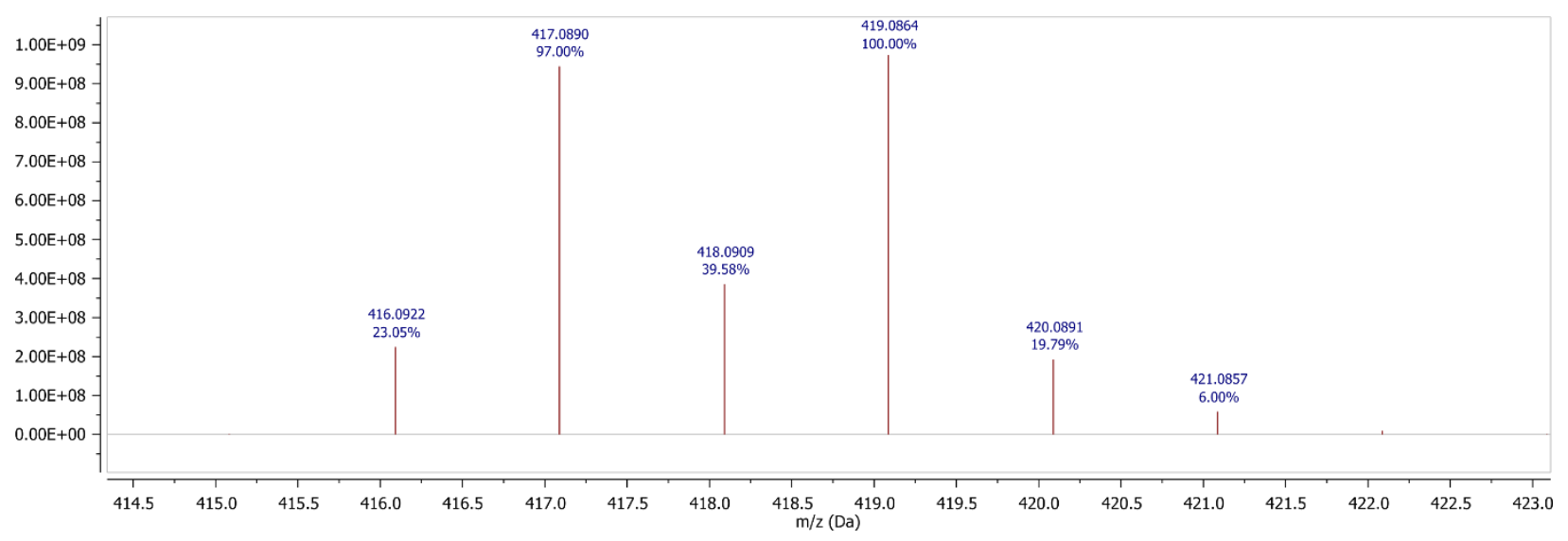

Figure S35. DART-MS of hexyl 2-bromo-5-(4,4,5,5-tetramethyl-1,3,2-dioxaborolan-2yl)thiophene-3-carboxylate (Compound 3a). 


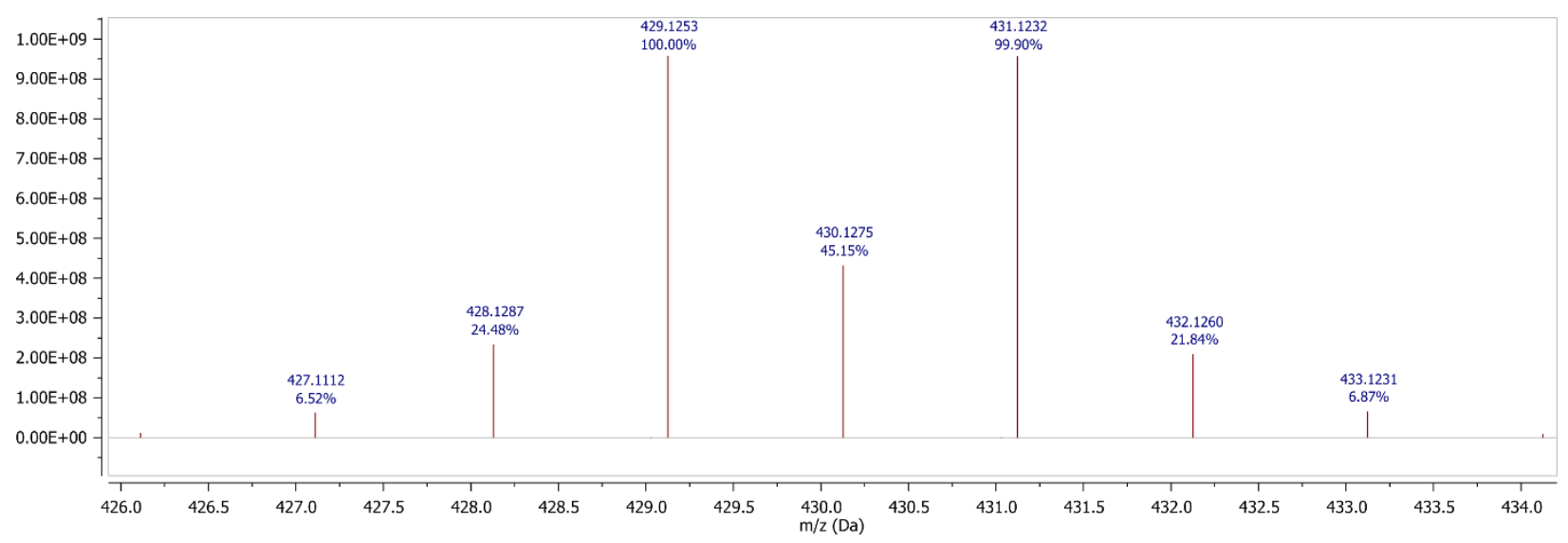

Figure S36. DART-MS of 1-(2-bromo-5-(4,4,5,5-tetramethyl-1,3,2-dioxaborolan-2yl)thiophen-3-yl)nonan-1-one (Compound 3b).

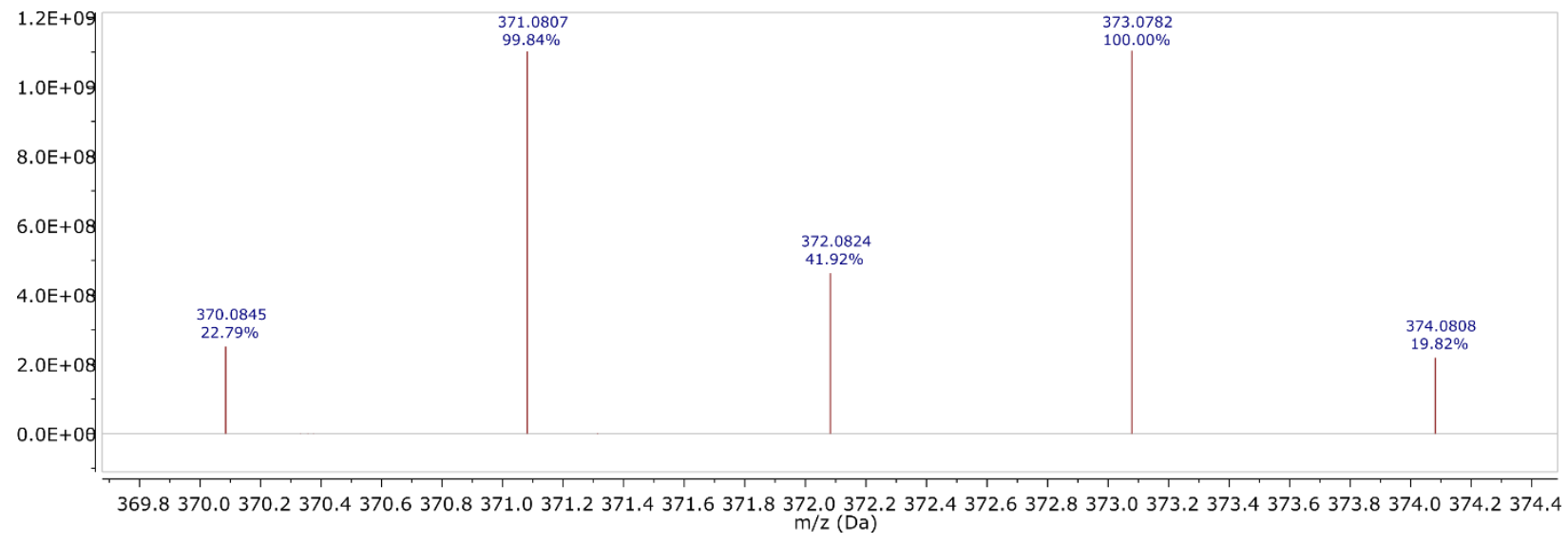

Figure S37. DART-MS of 2-(7-bromo-9H-fluoren-2-yl)-4,4,5,5-tetramethyl-1,3,2dioxaborolane (Compound $3 \mathrm{c}$ ). 


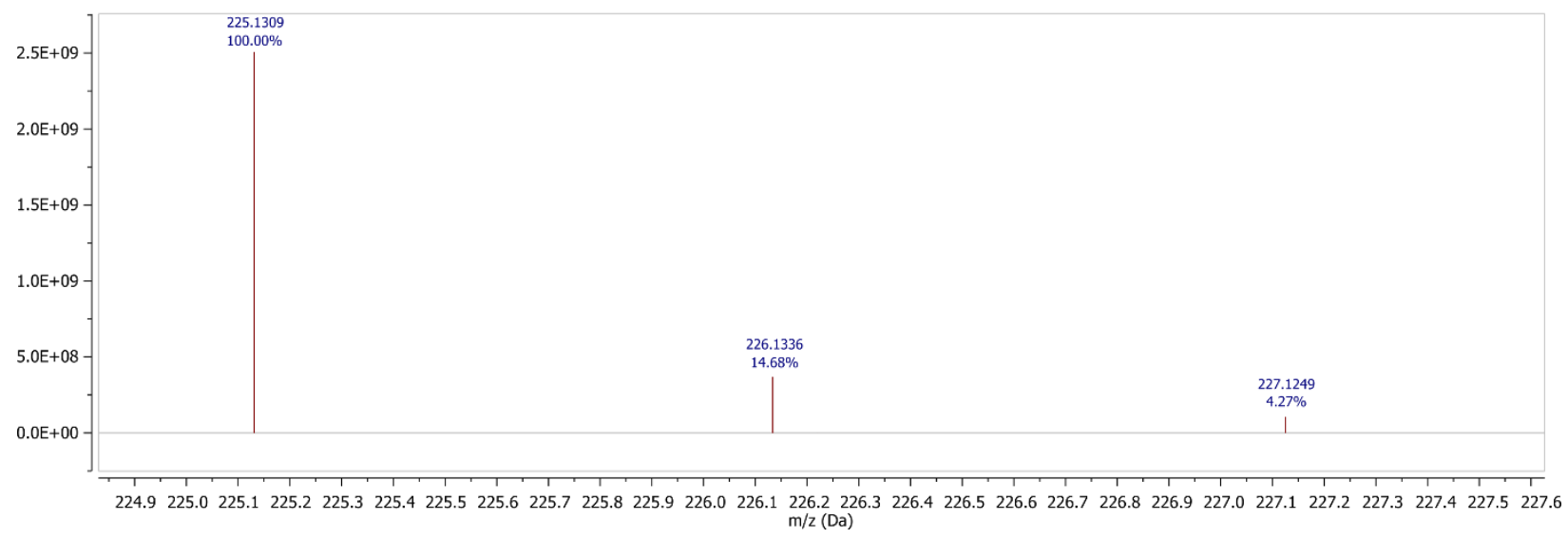

Figure S38. DART-MS of 1-(thiophen-3-yl)nonan-1-one.

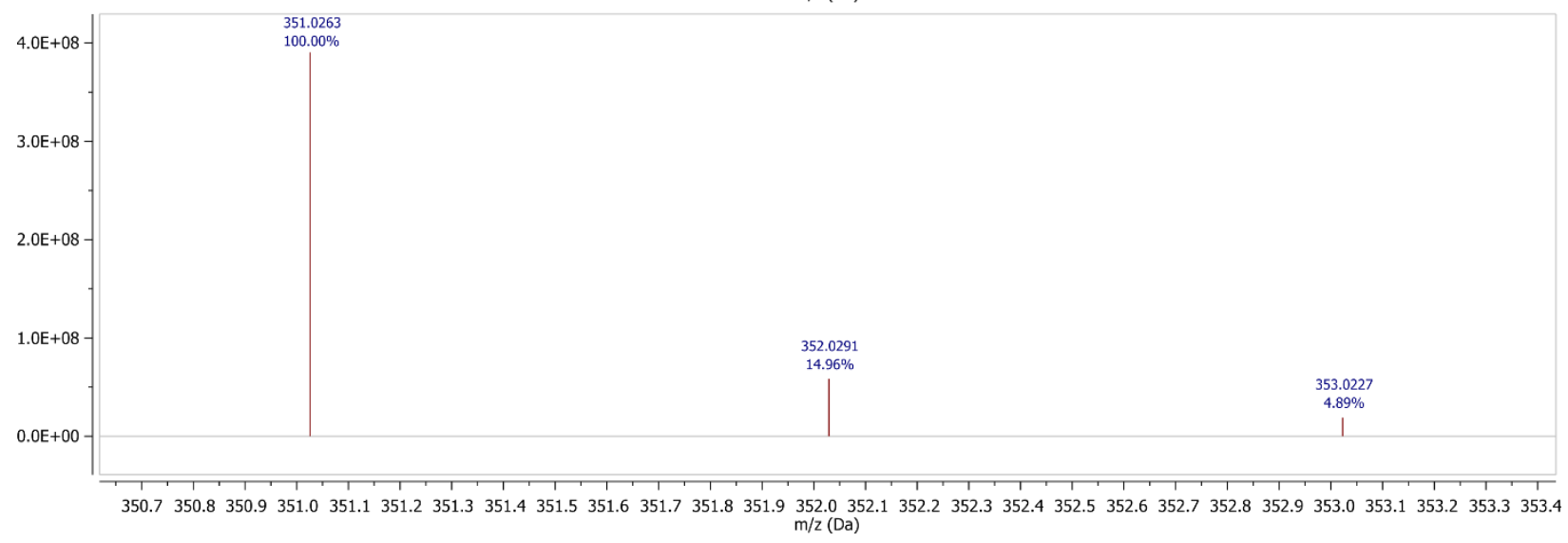

Figure S39. DART-MS of 1-(5-iodothiophen-3-yl)nonan-1-one. 


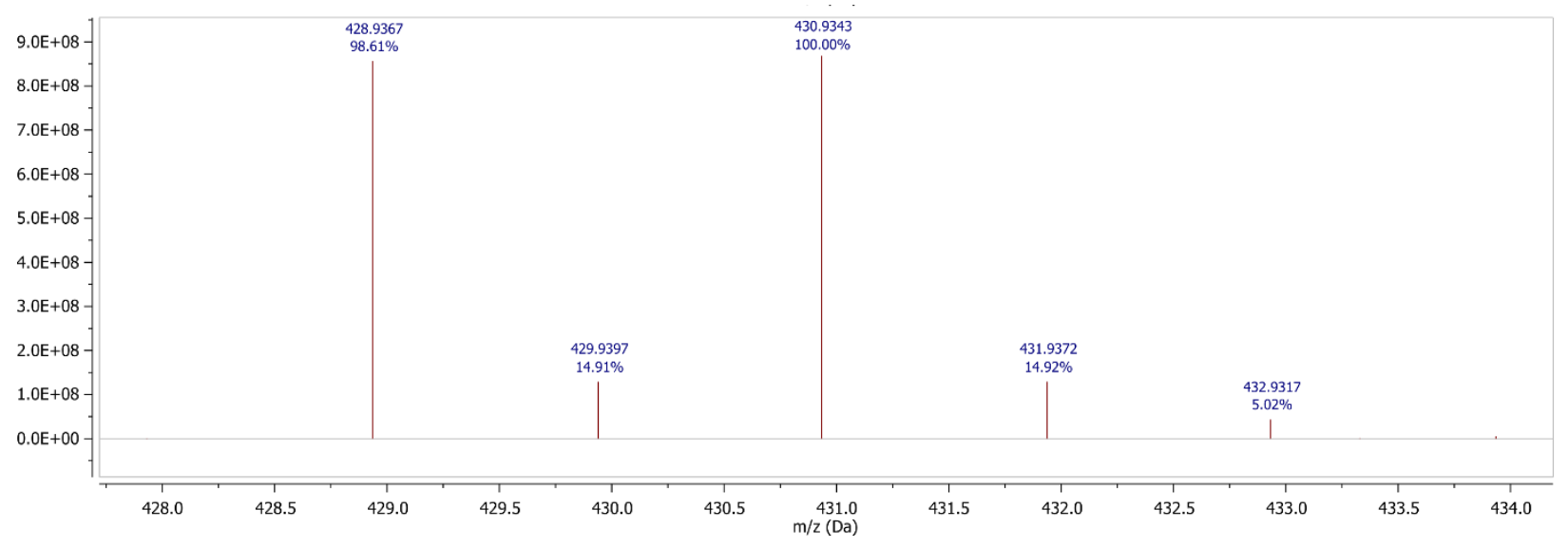

Figure S40. DART-MS of 1-(2-bromo-5-iodothiophen-3-yl)nonan-1-one.

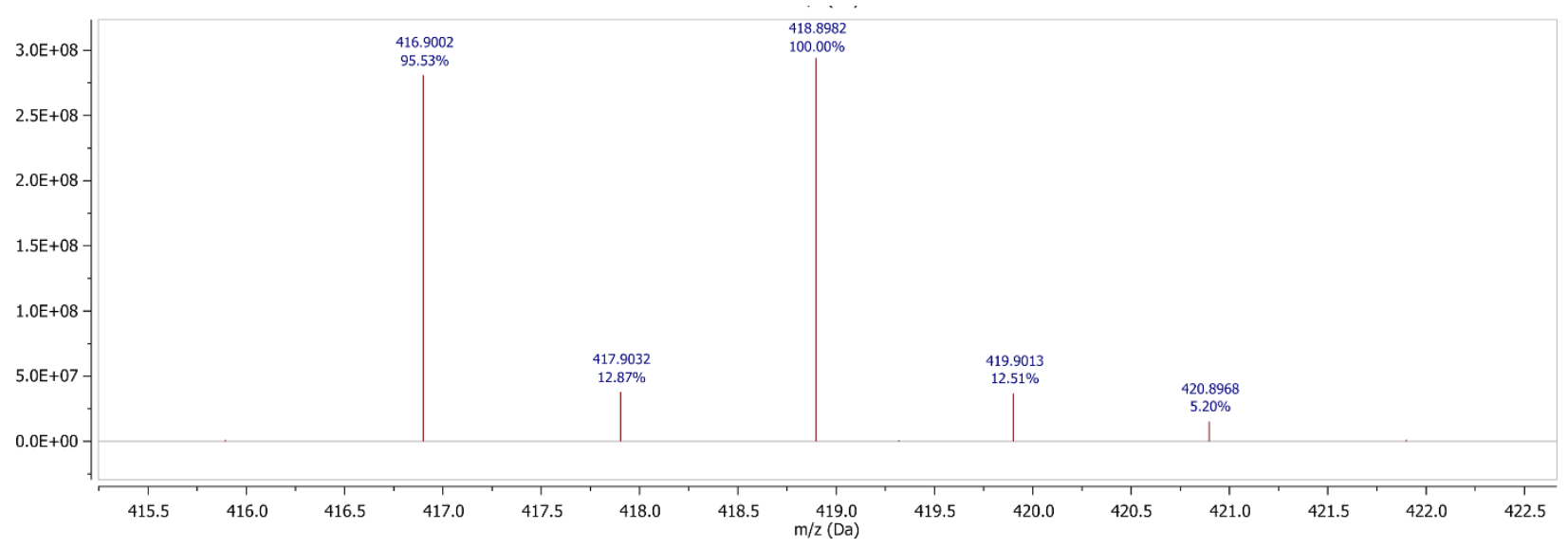

Figure S41. DART-MS of hexyl 2-bromo-5-iodothiophene-3-carboxylate. 
GC-MS Data for Conversion
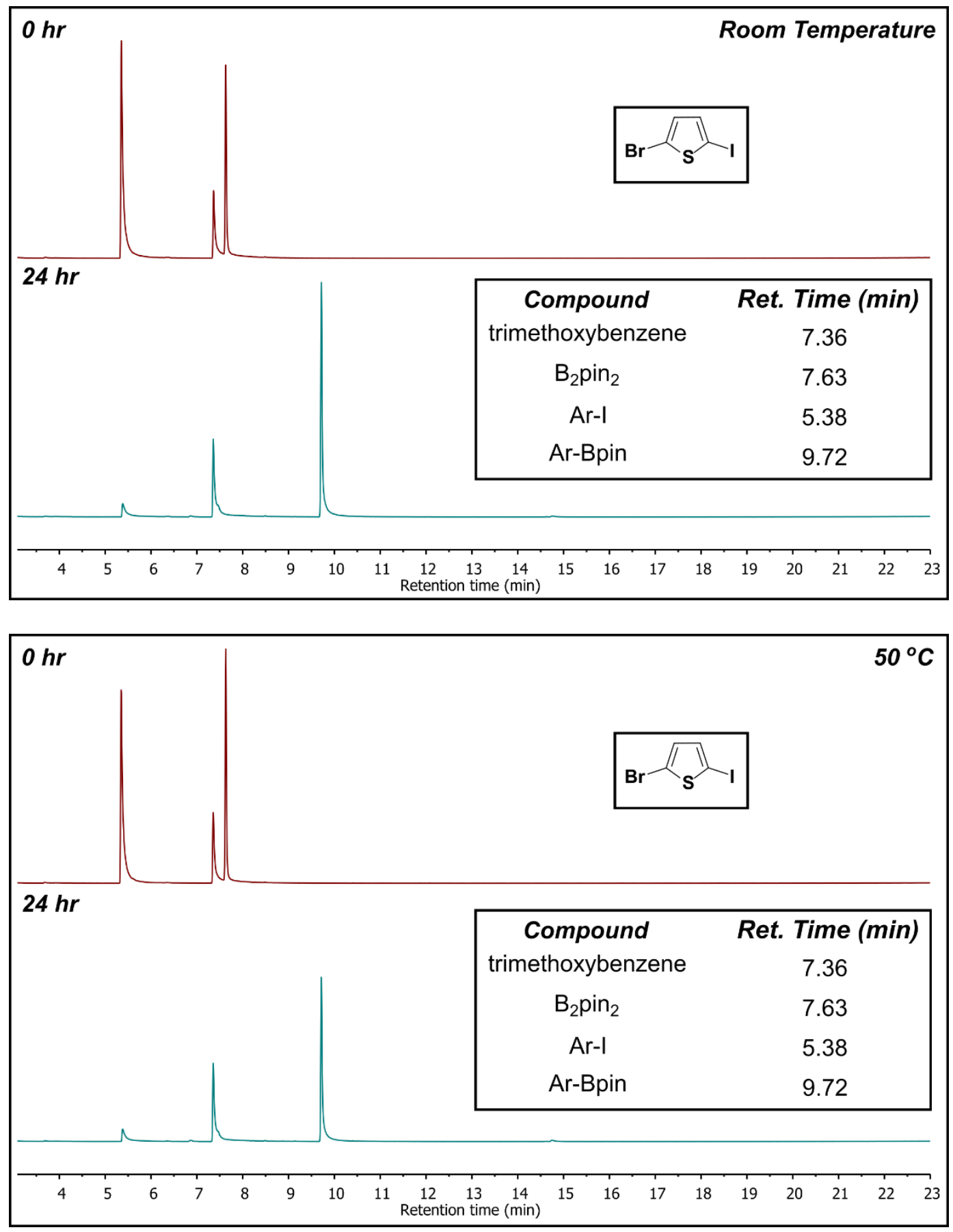

Figure S42. GC-MS spectra for conversion of 2-bromo-5-iodothiophene to 2-(5bromothiophen-2-yl)-4,4,5,5-tetramethyl-1,3,2-dioxaborolane at room temperature (top) and $50{ }^{\circ} \mathrm{C}$ (bottom). 

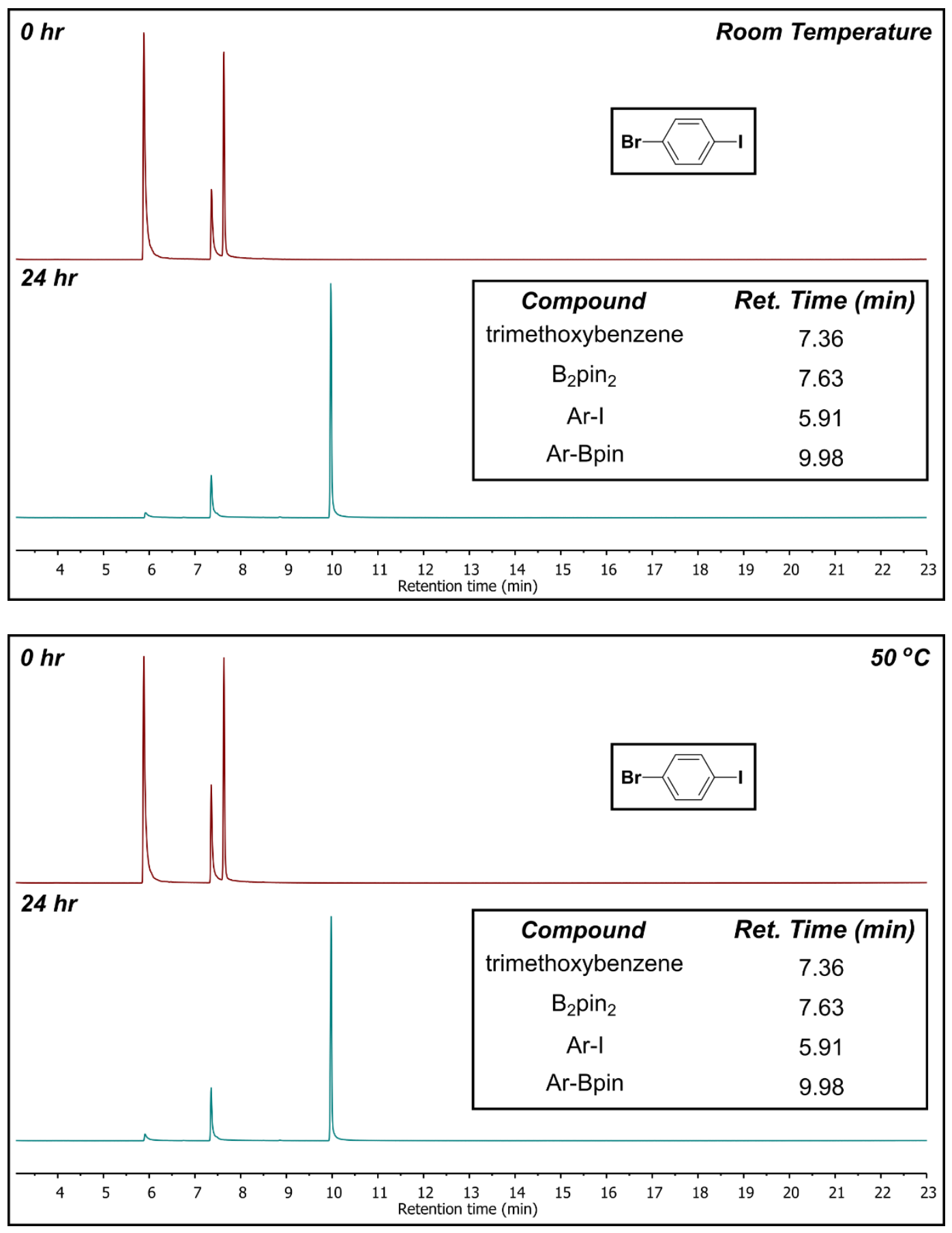

Figure S43. GC-MS spectra for conversion of 1-bromo-4-iodobenzene to 2-(4-bromophenyl)4,4,5,5-tetramethyl-1,3,2-dioxaborolane at room temperature (top) and $50{ }^{\circ} \mathrm{C}$ (bottom). 

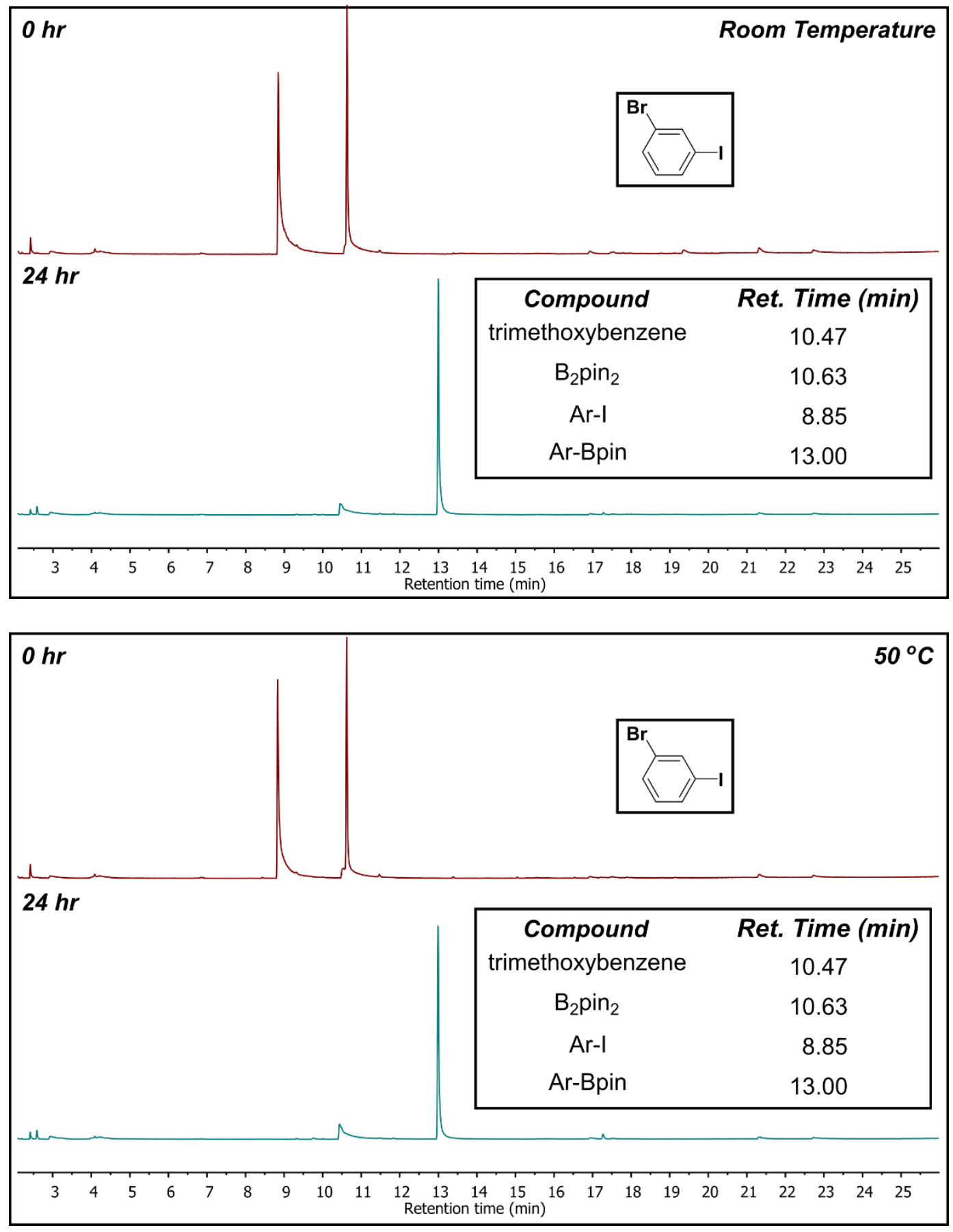

Figure S44. GC-MS spectra for conversion of 1-bromo-3-iodobenzene to 2-(3-bromophenyl)4,4,5,5-tetramethyl-1,3,2-dioxaborolane at room temperature (top) and $50{ }^{\circ} \mathrm{C}$ (bottom). 

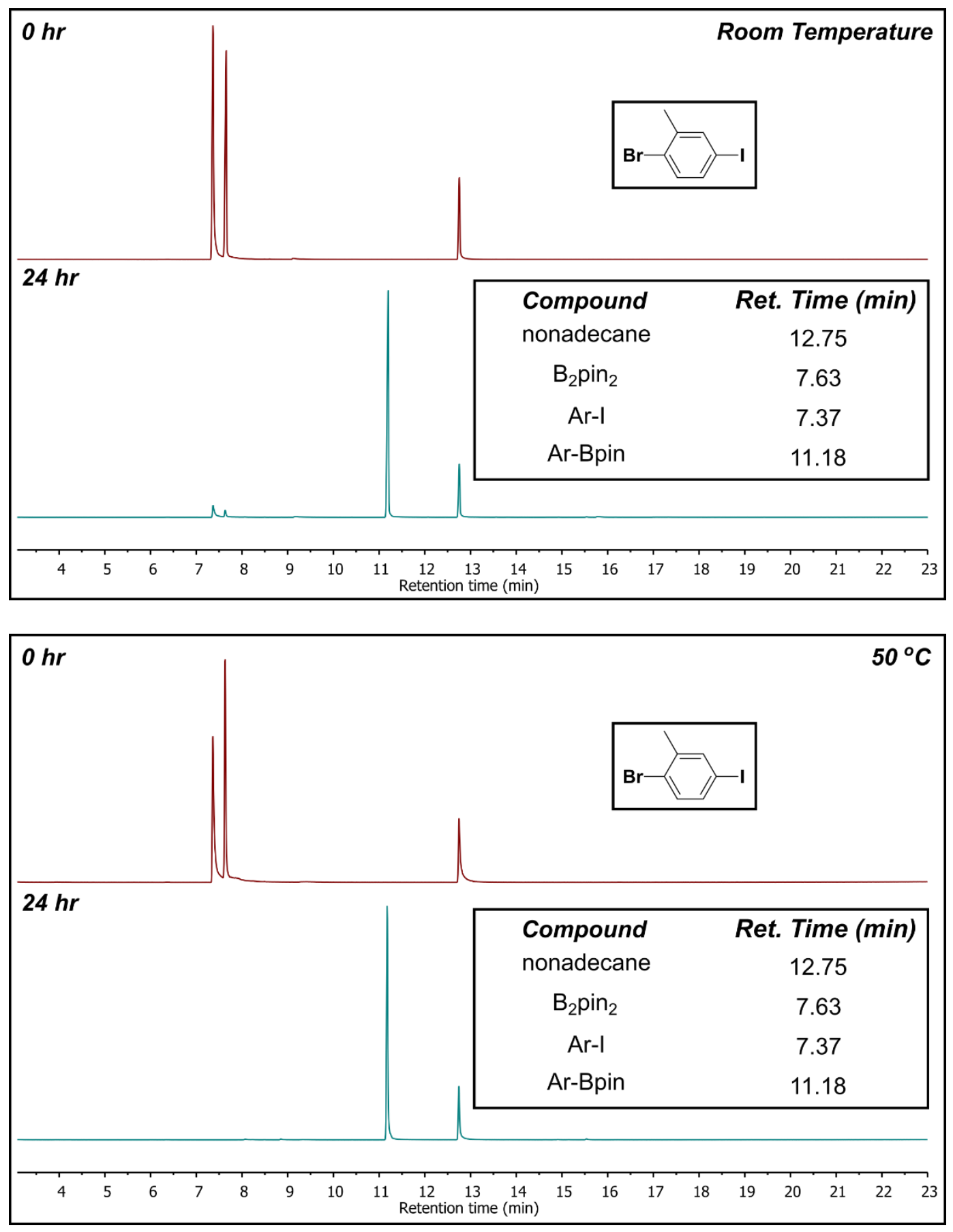

Figure S45. GC-MS spectra for conversion of 1-bromo-4-iodo-2-methylbenzene to 2-(4-bromo3-methylphenyl)-4,4,5,5-tetramethyl-1,3,2-dioxaborolane at room temperature (top) and $50{ }^{\circ} \mathrm{C}$ (bottom). 

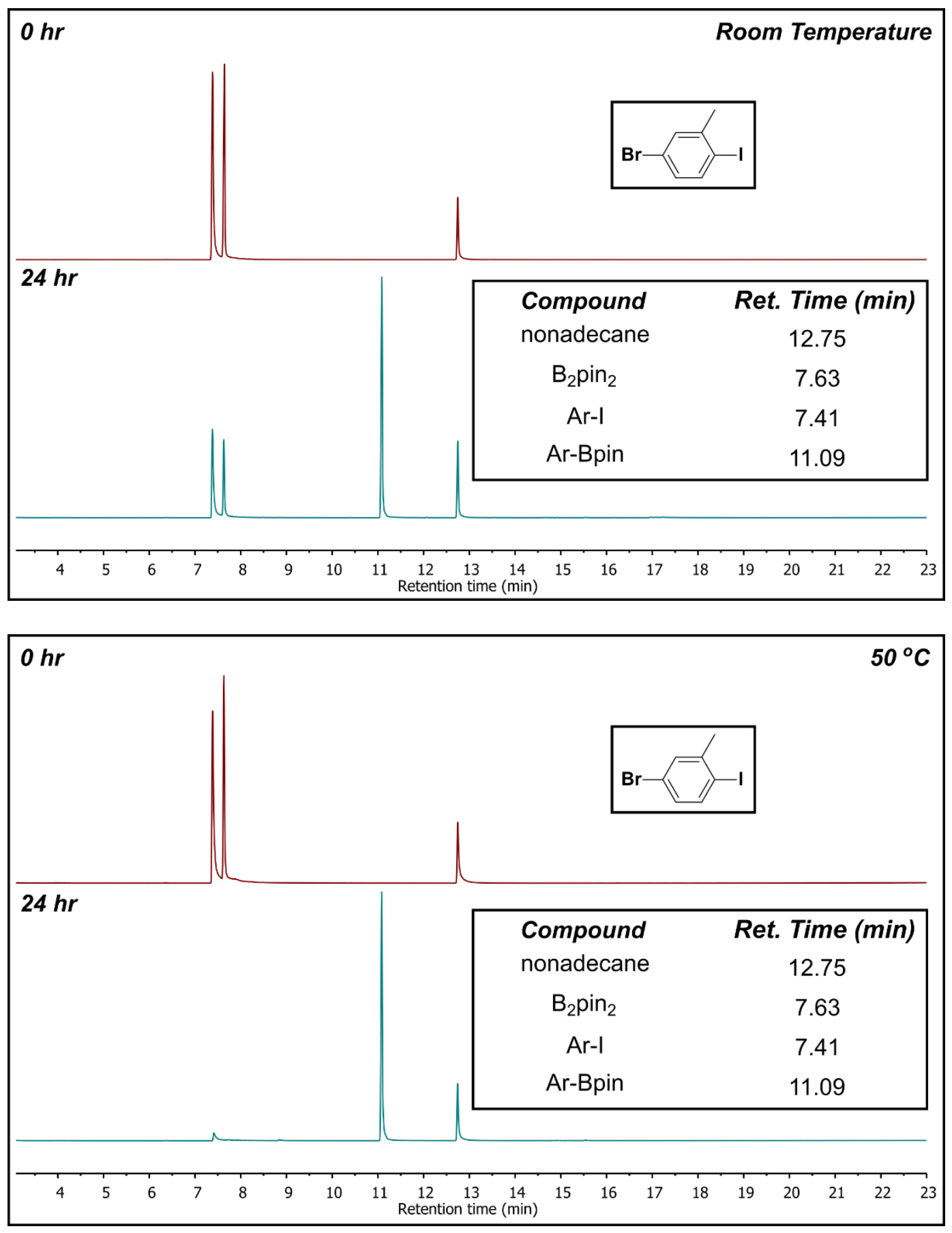

Figure S46. GC-MS spectra for conversion of 4-bromo-1-iodo-2-methylbenzene to 2-(4-bromo2-methylphenyl)-4,4,5,5-tetramethyl-1,3,2-dioxaborolane at room temperature (top) and $50{ }^{\circ} \mathrm{C}$ (bottom). 

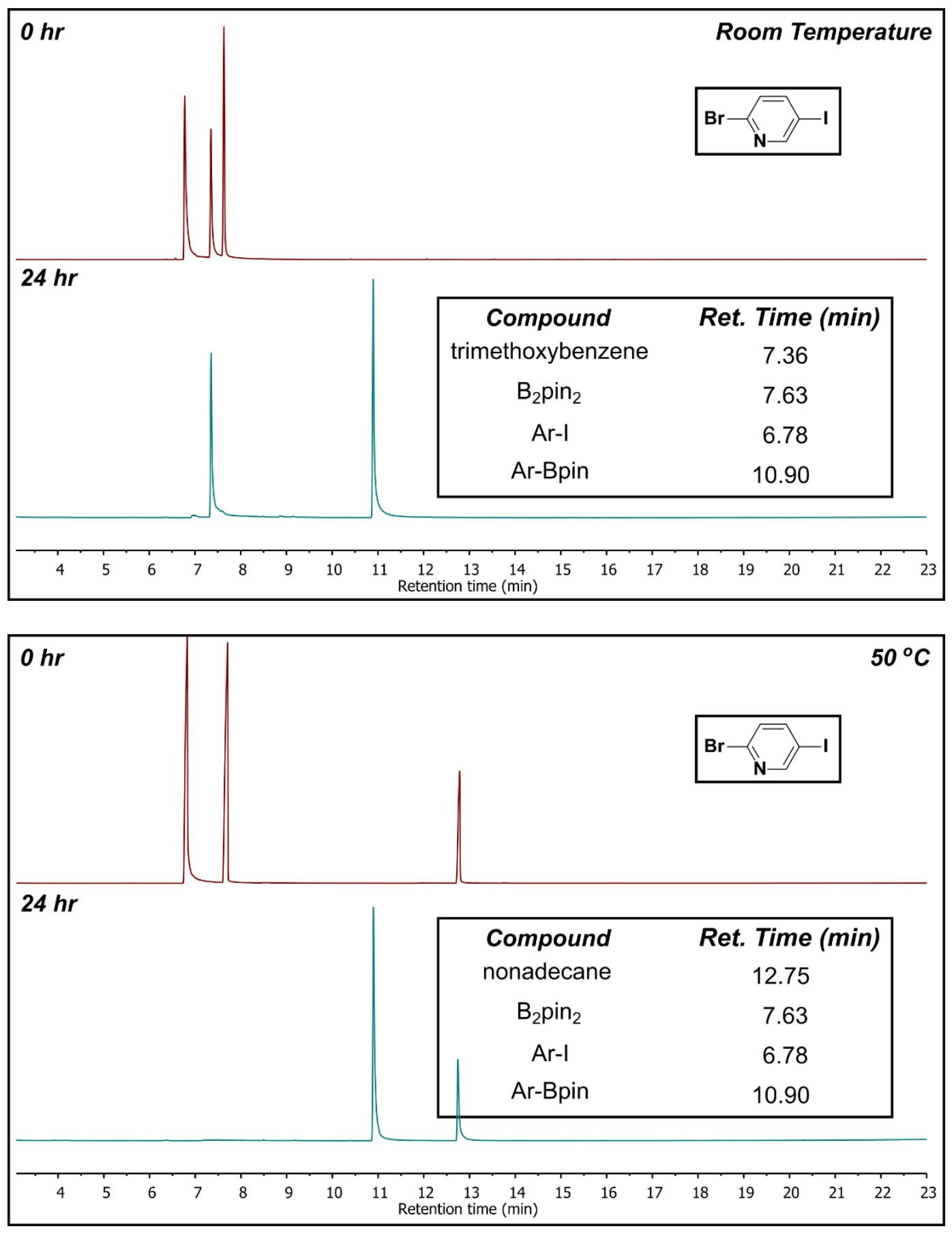

Figure S47. GC-MS spectra for conversion of 2-bromo-5-iodopyridine to 2-bromo-5-(4,4,5,5tetramethyl-1,3,2-dioxaborolan-2-yl)pyridine at room temperature (top) and $50{ }^{\circ} \mathrm{C}$ (bottom). 

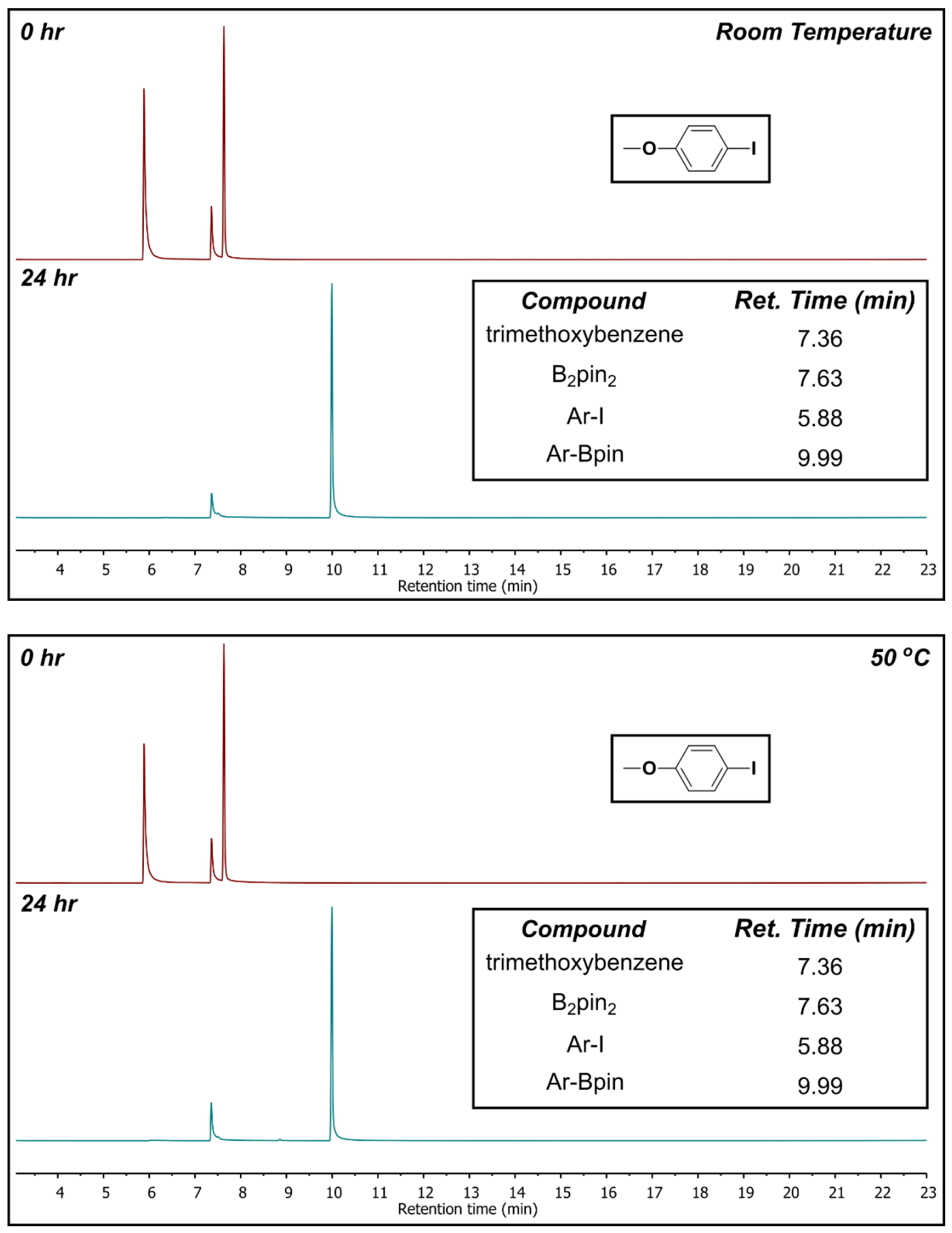

Figure S48. GC-MS spectra for conversion of 1-iodo-4-methoxybenzene to 2-(4methoxyphenyl)-4,4,5,5-tetramethyl-1,3,2-dioxaborolane at room temperature (top) and $50{ }^{\circ} \mathrm{C}$ (bottom). 

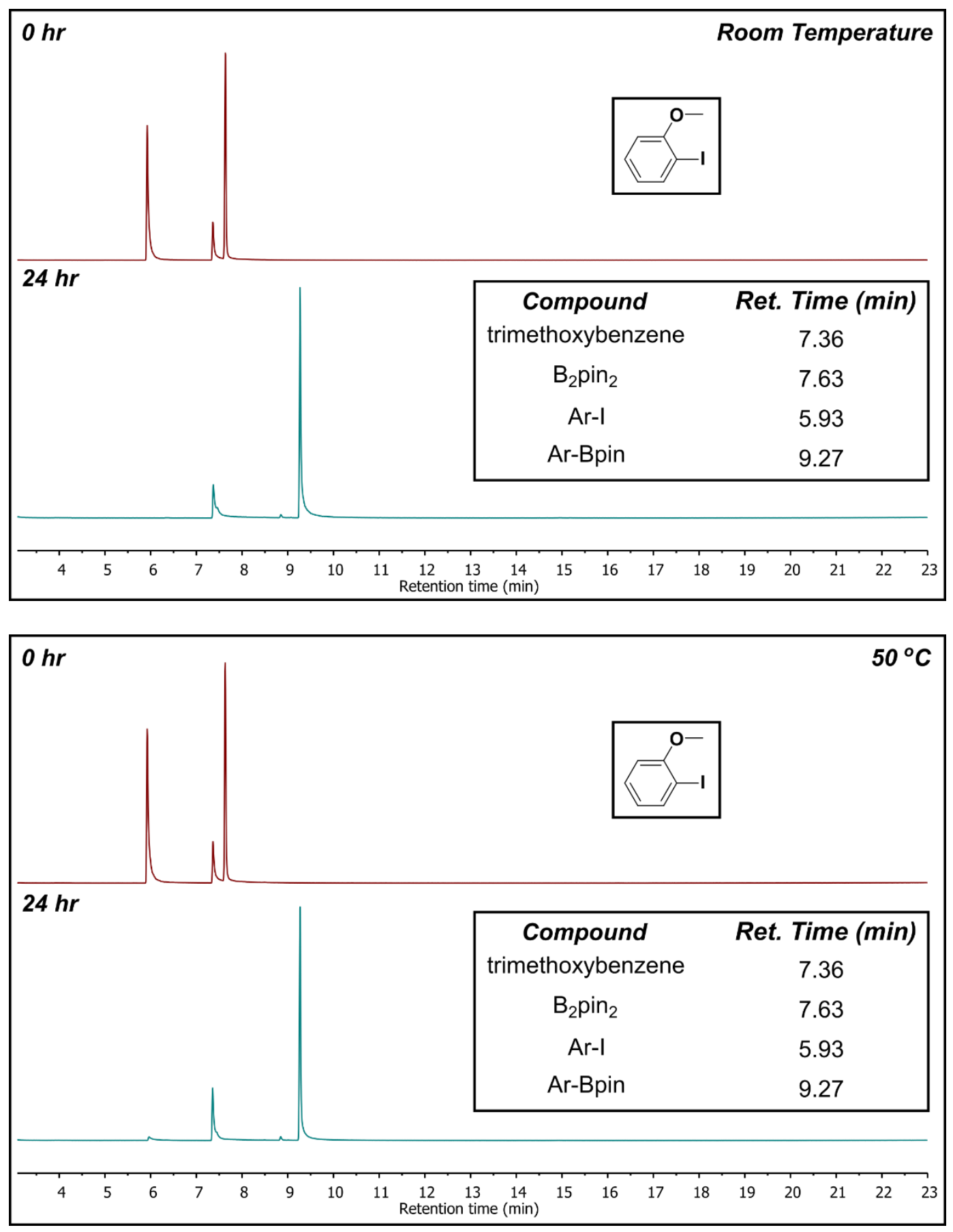

Figure S49. GC-MS spectra for conversion of 1-iodo-2-methoxybenzene to 2-(2methoxyphenyl)-4,4,5,5-tetramethyl-1,3,2-dioxaborolane at room temperature (top) and $50{ }^{\circ} \mathrm{C}$ (bottom). 

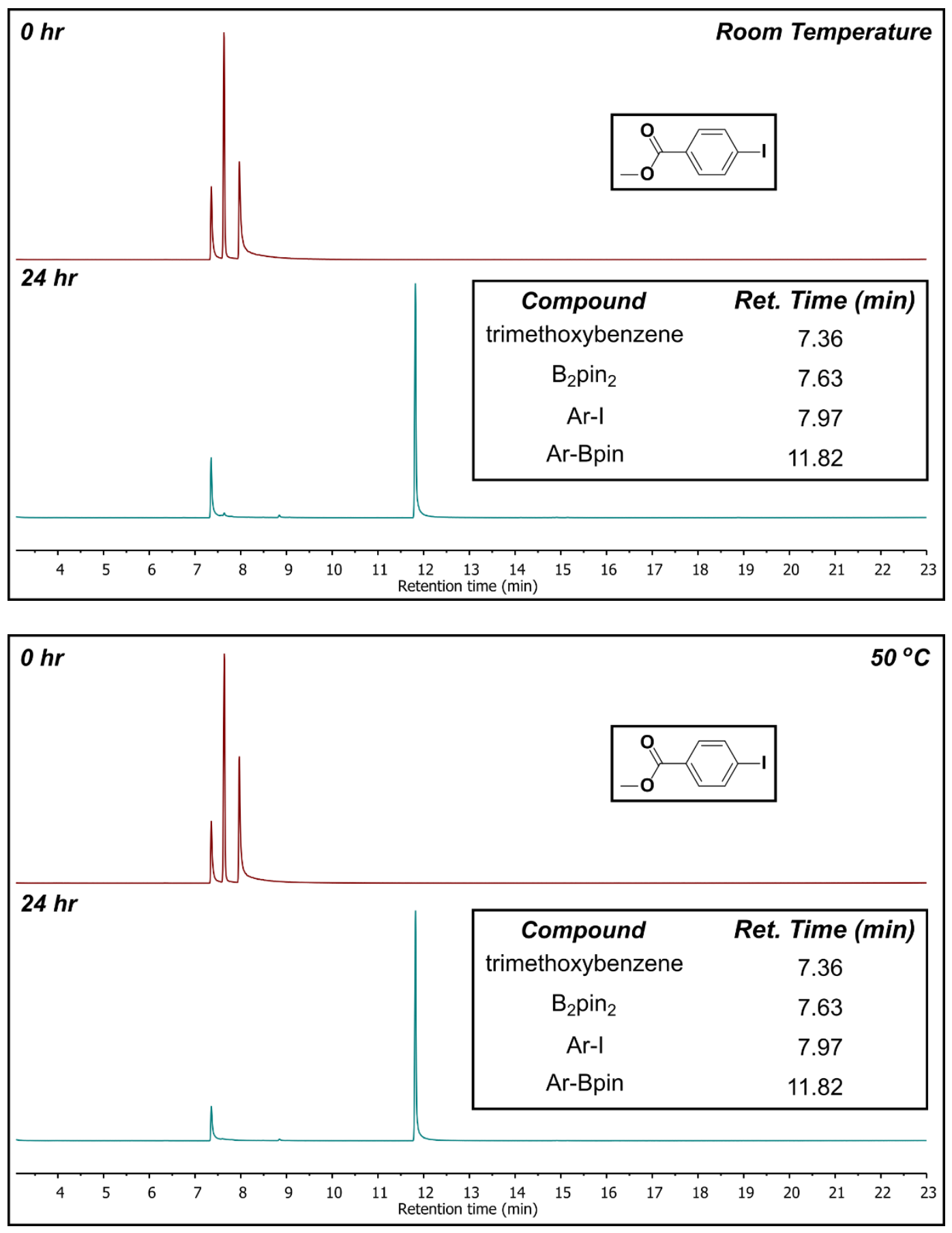

Figure S50. GC-MS spectra for conversion of methyl 4-iodobenzoate to methyl 4-(4,4,5,5tetramethyl-1,3,2-dioxaborolan-2-yl)benzoate at room temperature (top) and $50{ }^{\circ} \mathrm{C}$ (bottom). 

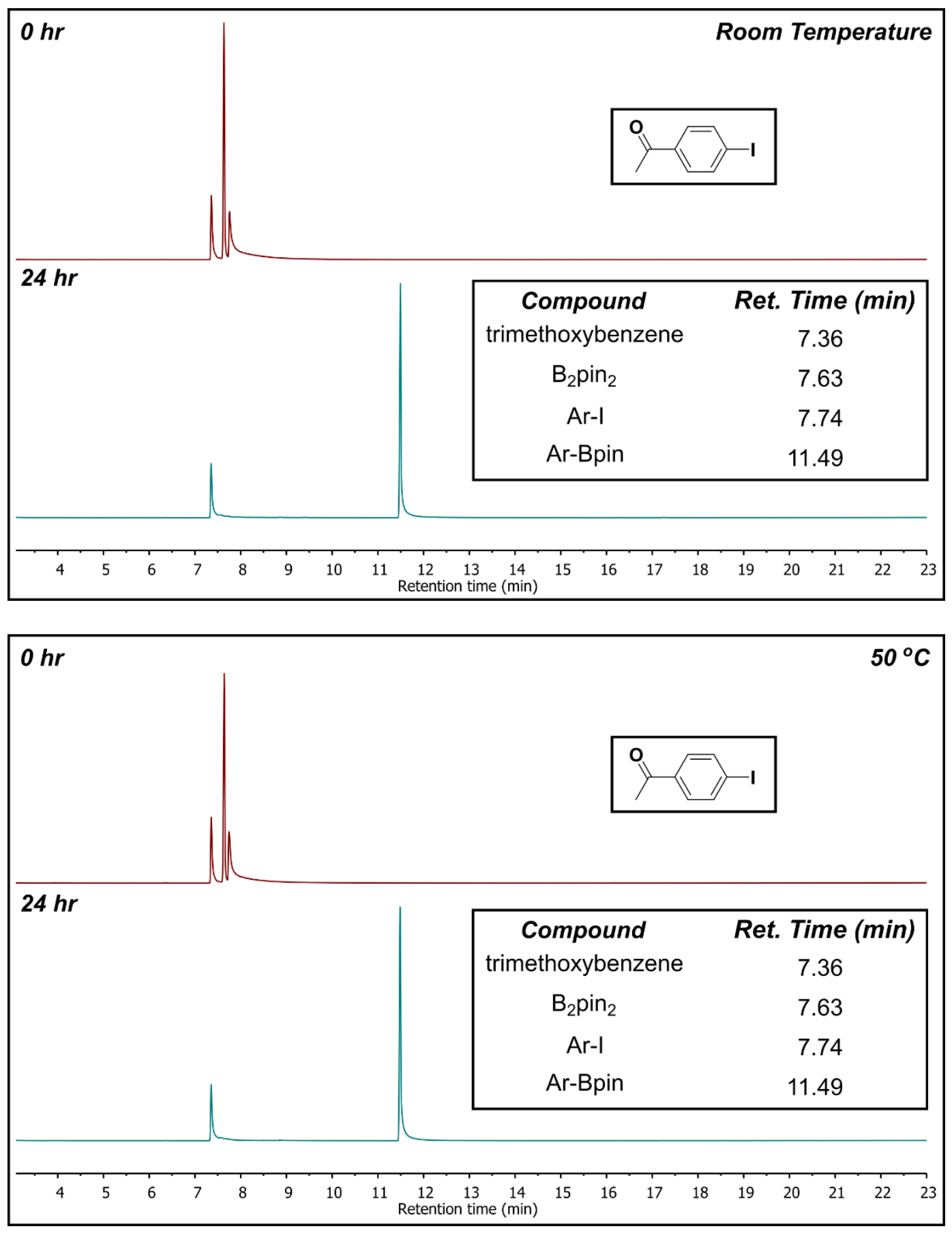

Figure S51. GC-MS spectra for conversion of 1-(4-iodophenyl)ethan-1-one to 1-(4-(4,4,5,5tetramethyl-1,3,2-dioxaborolan-2-yl)phenyl)ethan-1-one at room temperature (top) and $50{ }^{\circ} \mathrm{C}$ (bottom). 

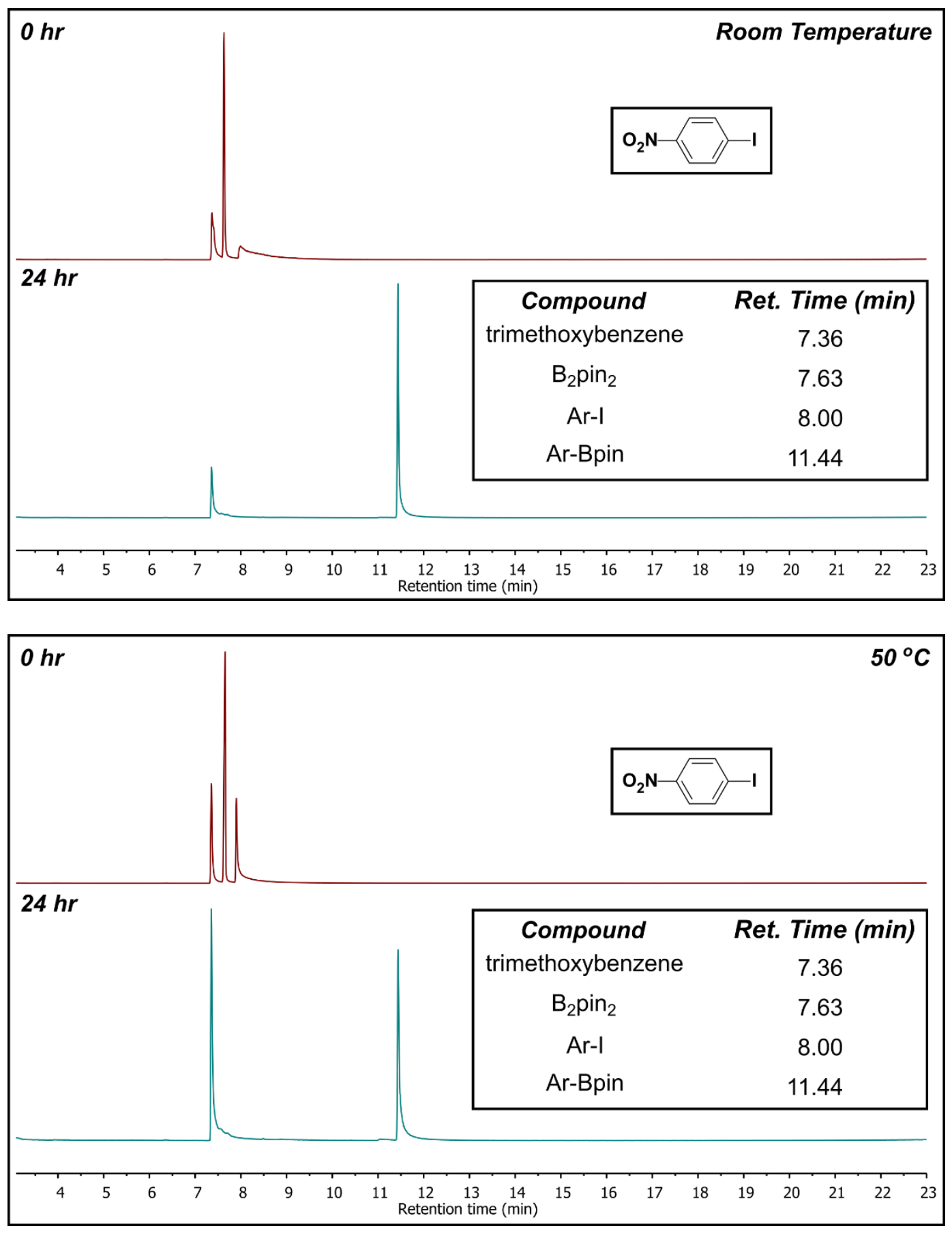

Figure S52. GC-MS spectra for conversion of 1-iodo-4-nitrobenzene to 4,4,5,5-tetramethyl-2(4-nitrophenyl)-1,3,2-dioxaborolane at room temperature (top) and $50{ }^{\circ} \mathrm{C}$ (bottom). 

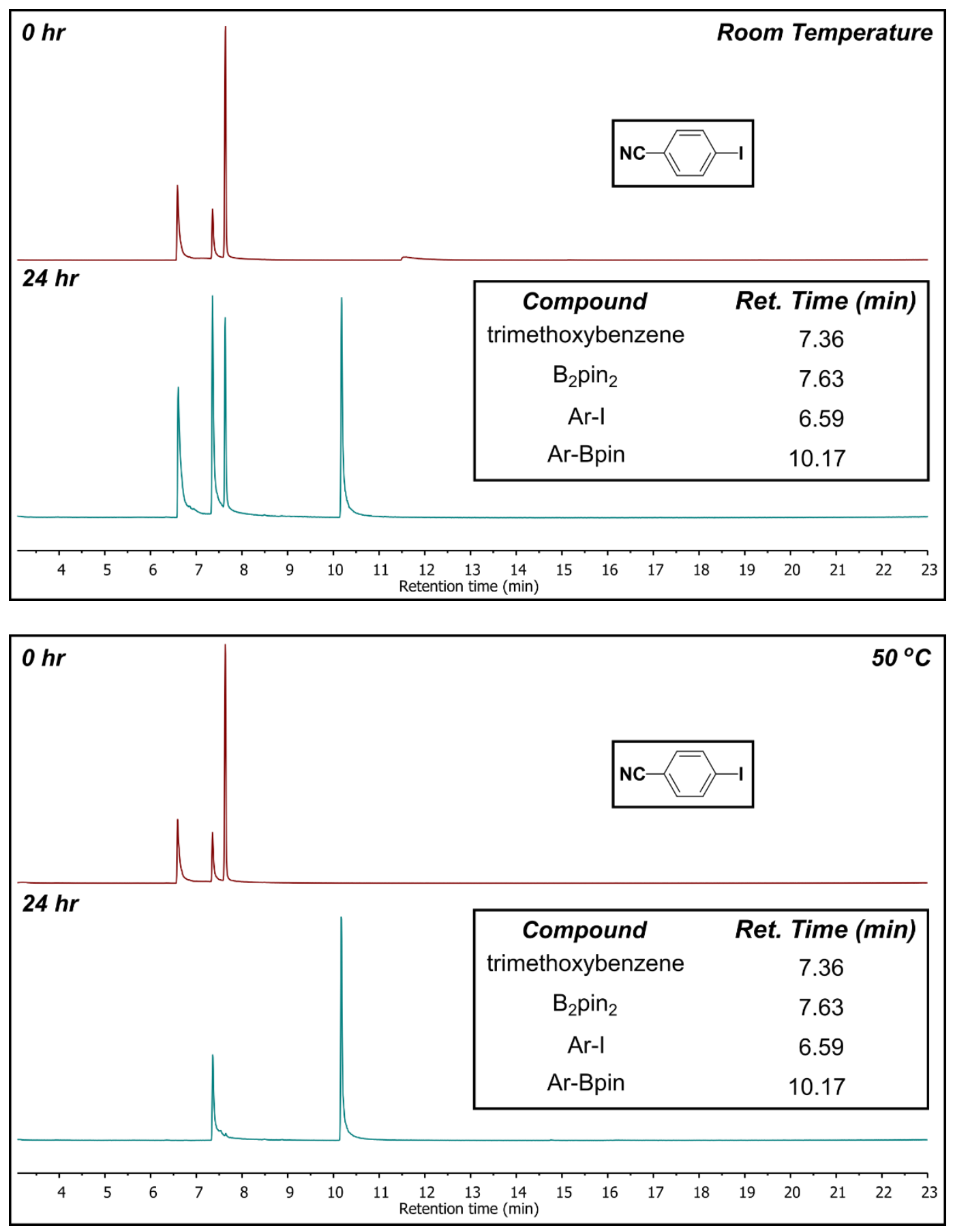

Figure S53. GC-MS spectra for conversion of 4-iodobenzonitrile to 4-(4,4,5,5-tetramethyl1,3,2-dioxaborolan-2-yl)benzonitrile at room temperature (top) and $50{ }^{\circ} \mathrm{C}$ (bottom). 

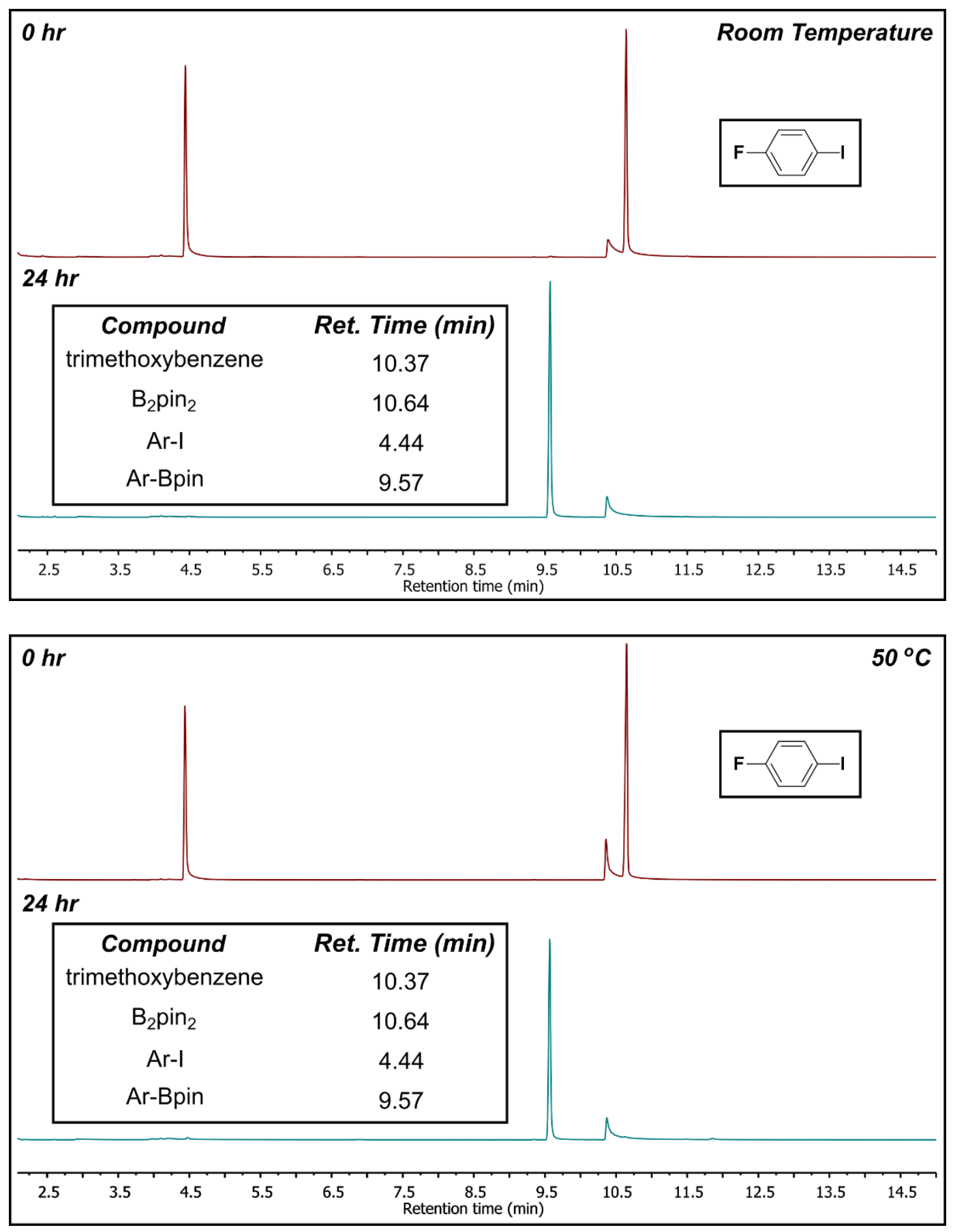

Figure S54. GC-MS spectra for conversion of 1-fluoro-4-iodobenzene to 2-(4-fluorophenyl)4,4,5,5-tetramethyl-1,3,2-dioxaborolane at room temperature (top) and $50{ }^{\circ} \mathrm{C}$ (bottom). 

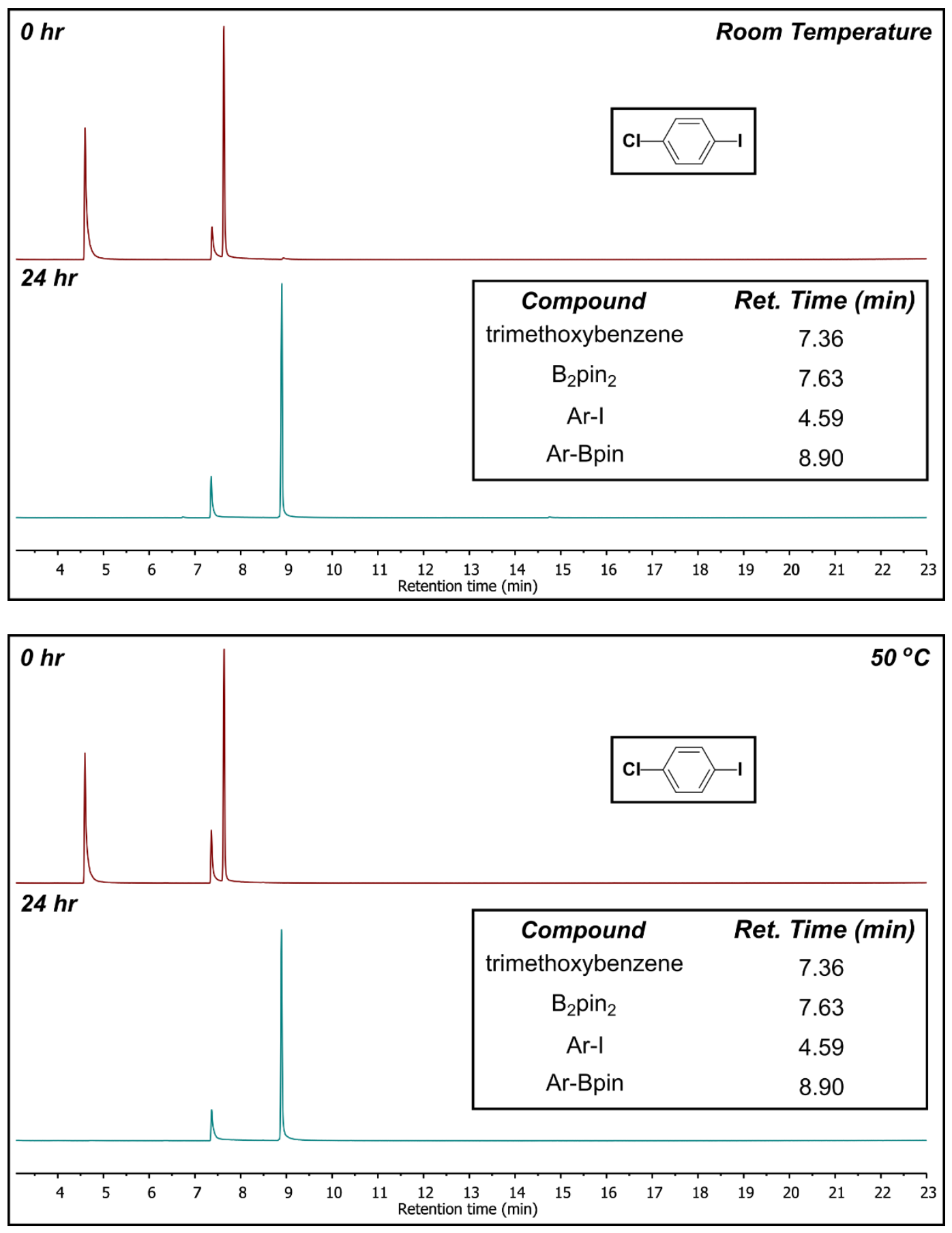

Figure S55. GC-MS spectra for conversion of 1-chloro-4-iodobenzene to 2-(4-chlorophenyl)4,4,5,5-tetramethyl-1,3,2-dioxaborolane at room temperature (top) and $50{ }^{\circ} \mathrm{C}$ (bottom). 

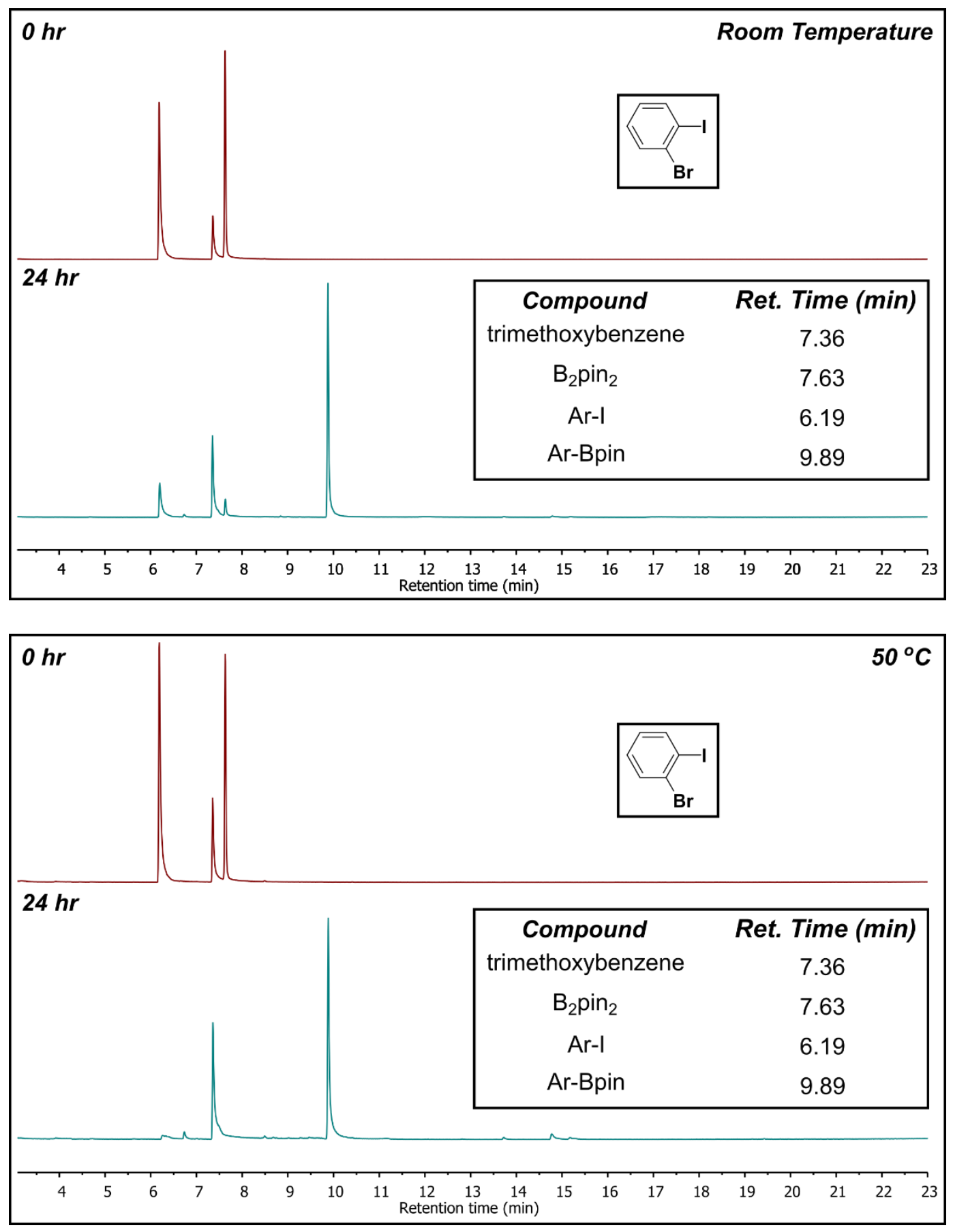

Figure S56. GC-MS spectra for conversion of 1-bromo-2-iodobenzene to 2-(2-bromophenyl)4,4,5,5-tetramethyl-1,3,2-dioxaborolane at room temperature (top) and $50{ }^{\circ} \mathrm{C}$ (bottom). 

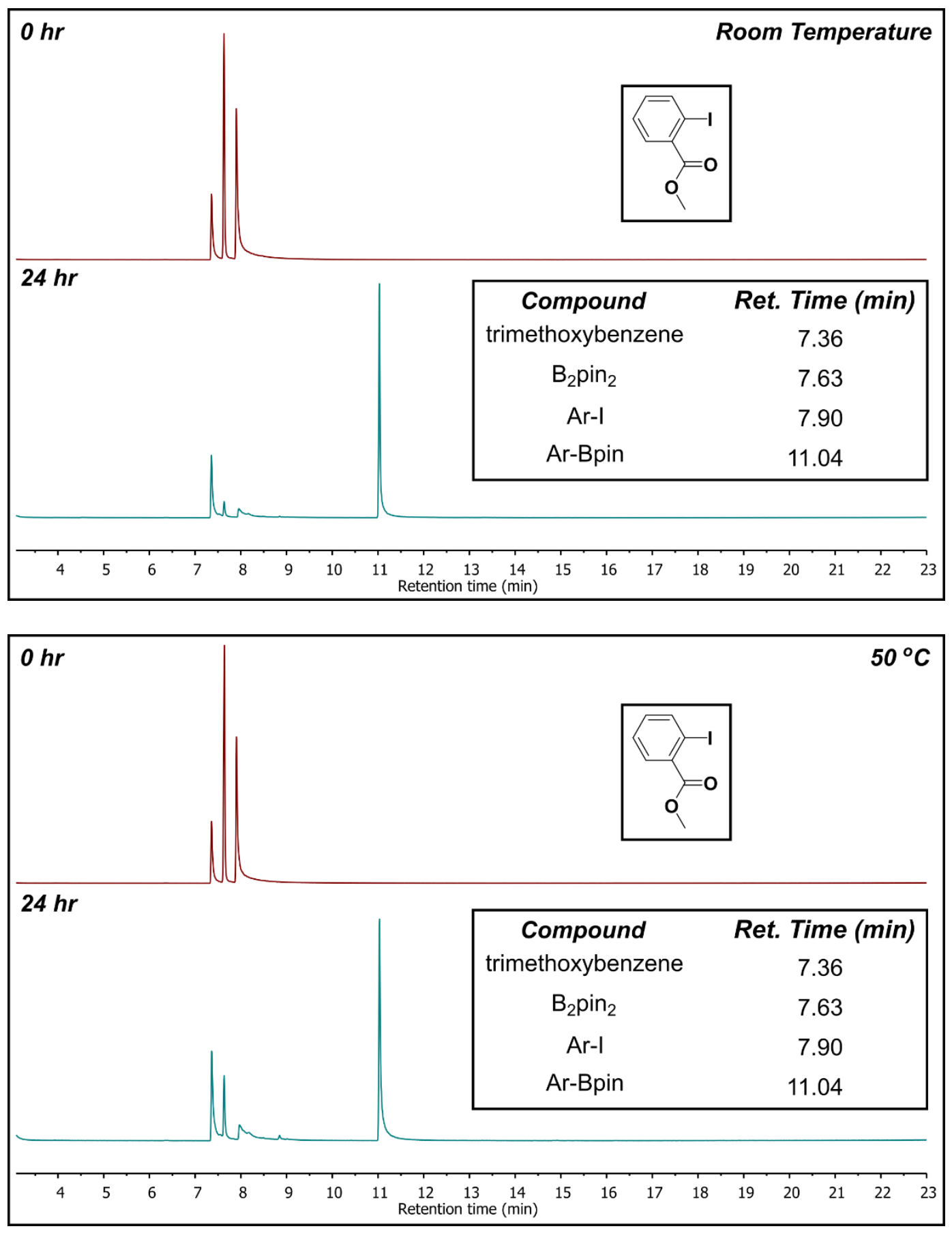

Figure S57. GC-MS spectra for conversion of methyl 2-iodobenzoate to methyl 2-(4,4,5,5tetramethyl-1,3,2-dioxaborolan-2-yl)benzoate at room temperature (top) and $50{ }^{\circ} \mathrm{C}$ (bottom). 

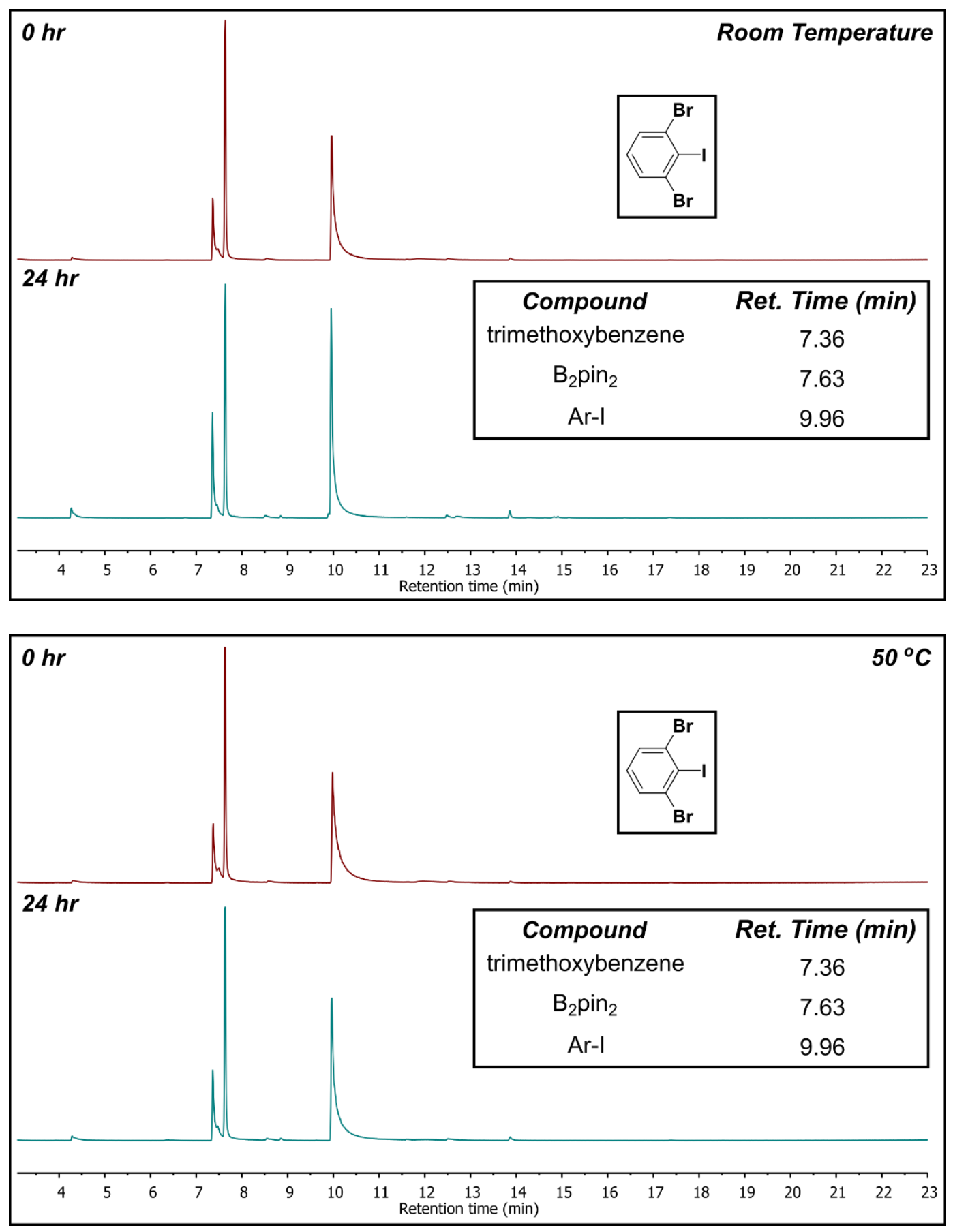

Figure S58. GC-MS spectra for conversion of 1,3-dibromo-2-iodobenzene to 2-(2,6dibromophenyl)-4,4,5,5-tetramethyl-1,3,2-dioxaborolane at room temperature (top) and $50{ }^{\circ} \mathrm{C}$ (bottom). 

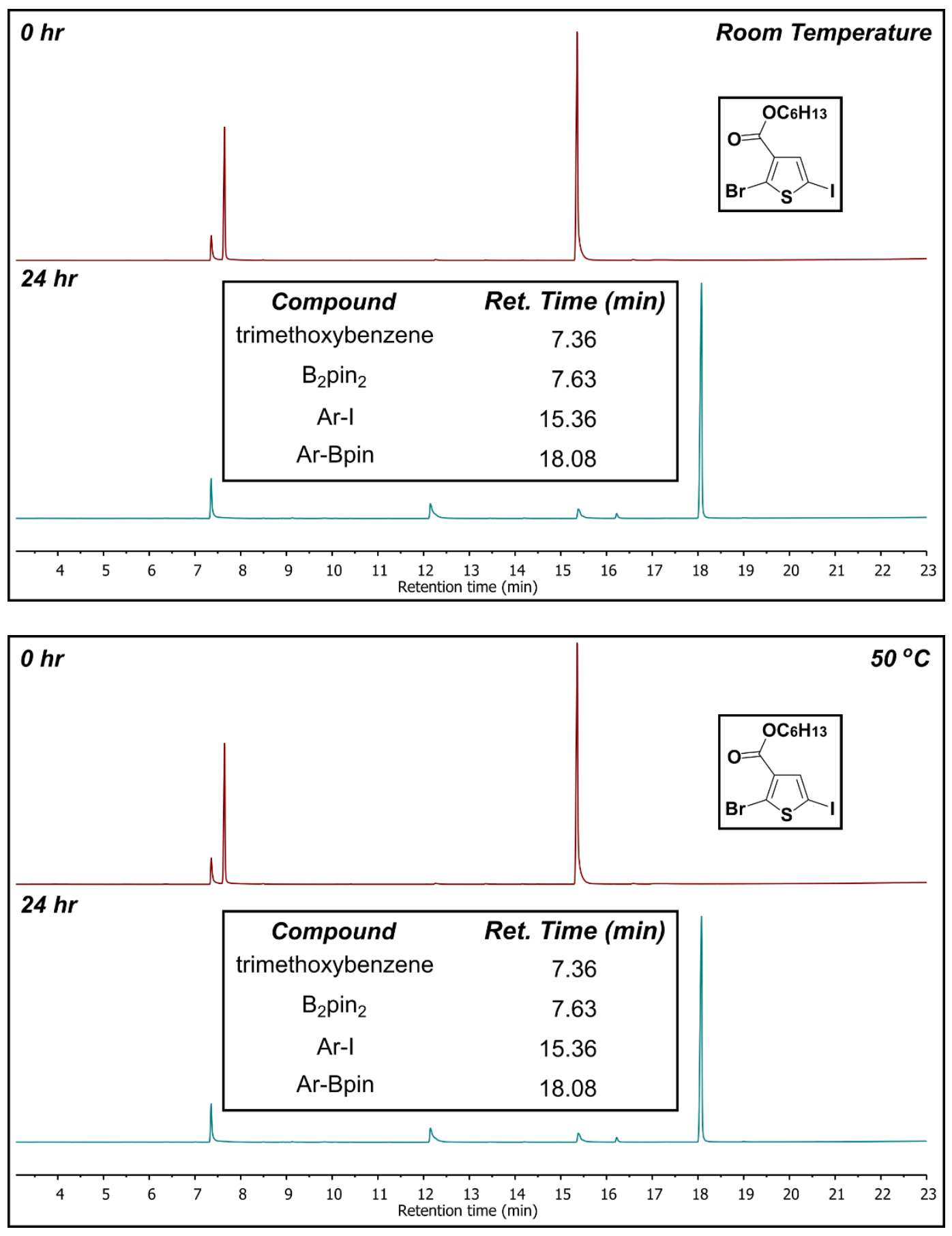

Figure S59. GC-MS spectra for conversion of hexyl 2-bromo-5-iodothiophene-3-carboxylate to hexyl 2-bromo-5-(4,4,5,5-tetramethyl-1,3,2-dioxaborolan-2-yl)thiophene-3-carboxylate at room temperature (top) and $50^{\circ} \mathrm{C}$ (bottom). 

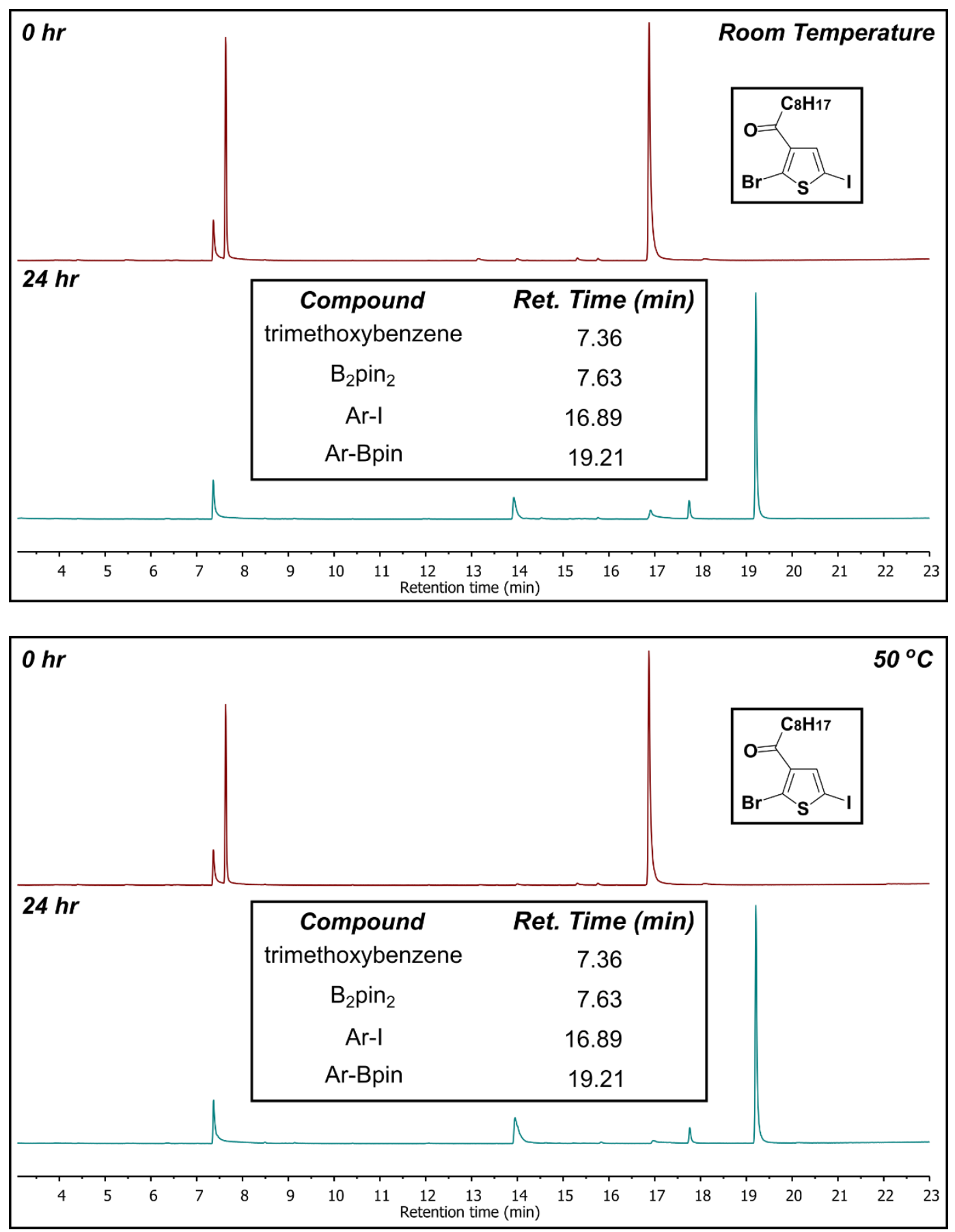

Figure S60. GC-MS spectra for conversion of 1-(2-bromo-5-iodothiophen-3-yl)nonan-1-one to 1-(2-bromo-5-(4,4,5,5-tetramethyl-1,3,2-dioxaborolan-2-yl)thiophen-3-yl)nonan-1-one at room temperature (top) and $50{ }^{\circ} \mathrm{C}$ (bottom). 

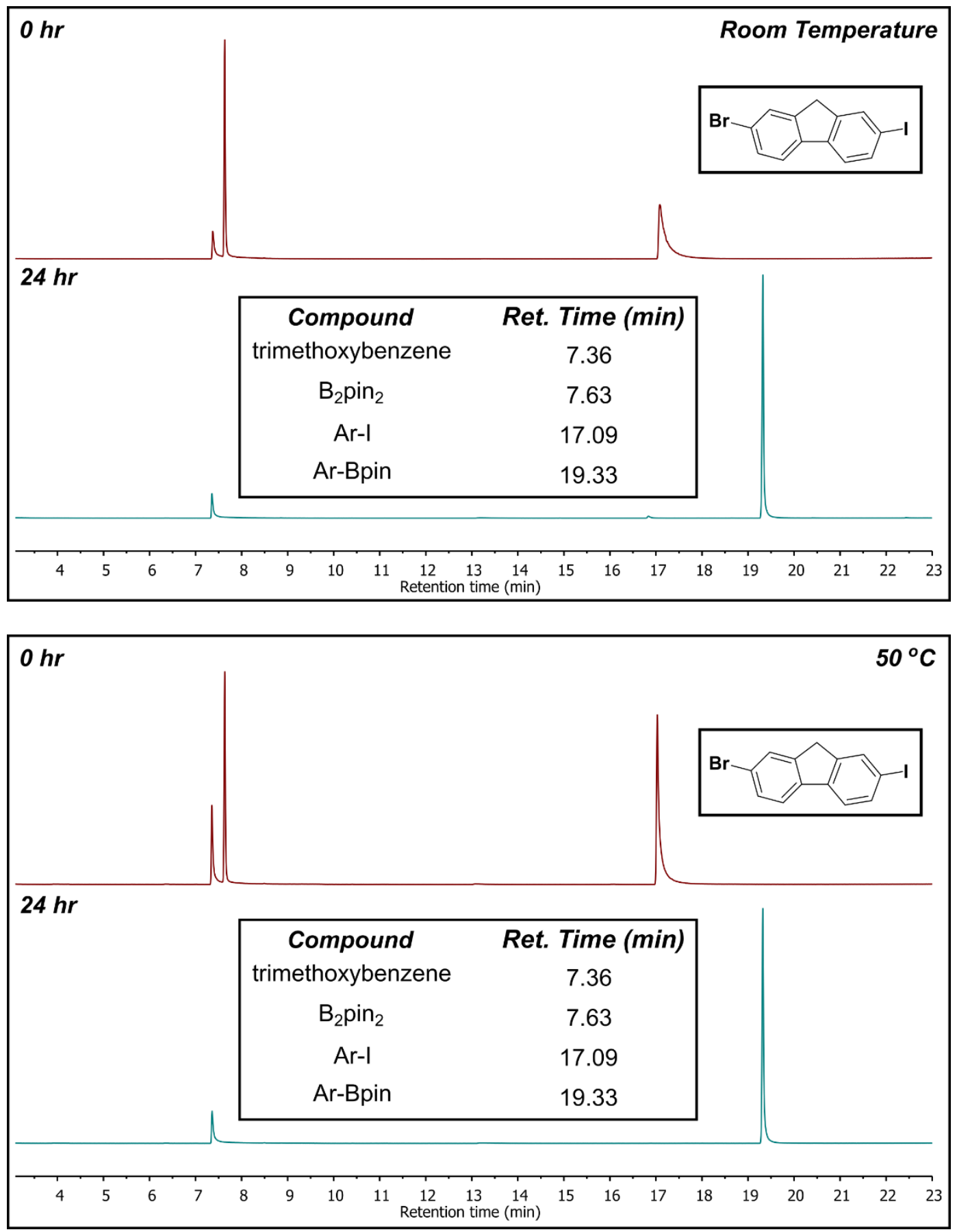

Figure S61. GC-MS spectra for conversion of 2-bromo-7-iodo-9H-fluorene to 2-(7-bromo-9Hfluoren-2-yl)-4,4,5,5-tetramethyl-1,3,2-dioxaborolane at room temperature (top) and $50{ }^{\circ} \mathrm{C}$ (bottom). 

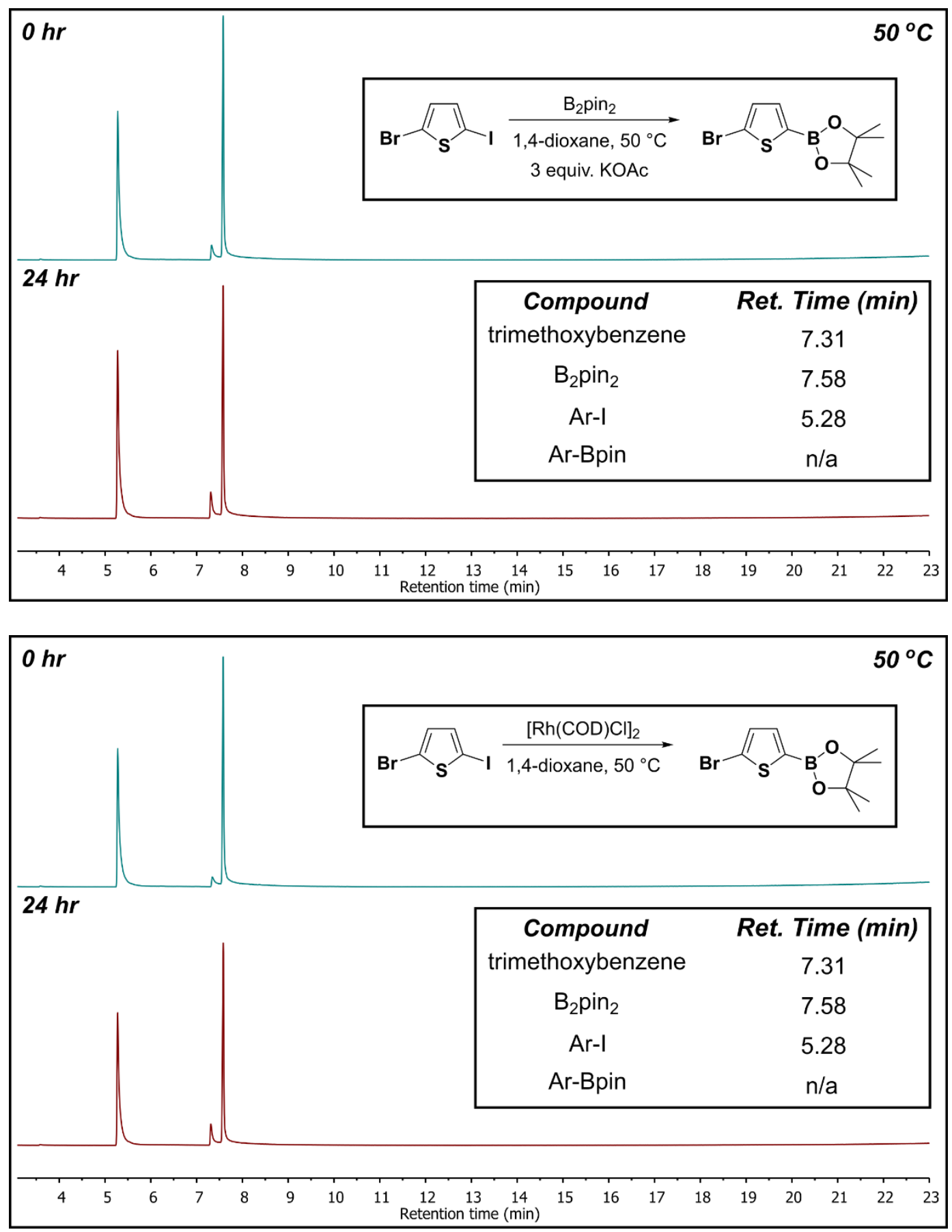

Figure S62. GC-MS spectra for control experiments performed under standard conditions without $[\mathrm{Rh}(\mathrm{COD}) \mathrm{Cl}]_{2}$ (top) and without KOAc (bottom). 

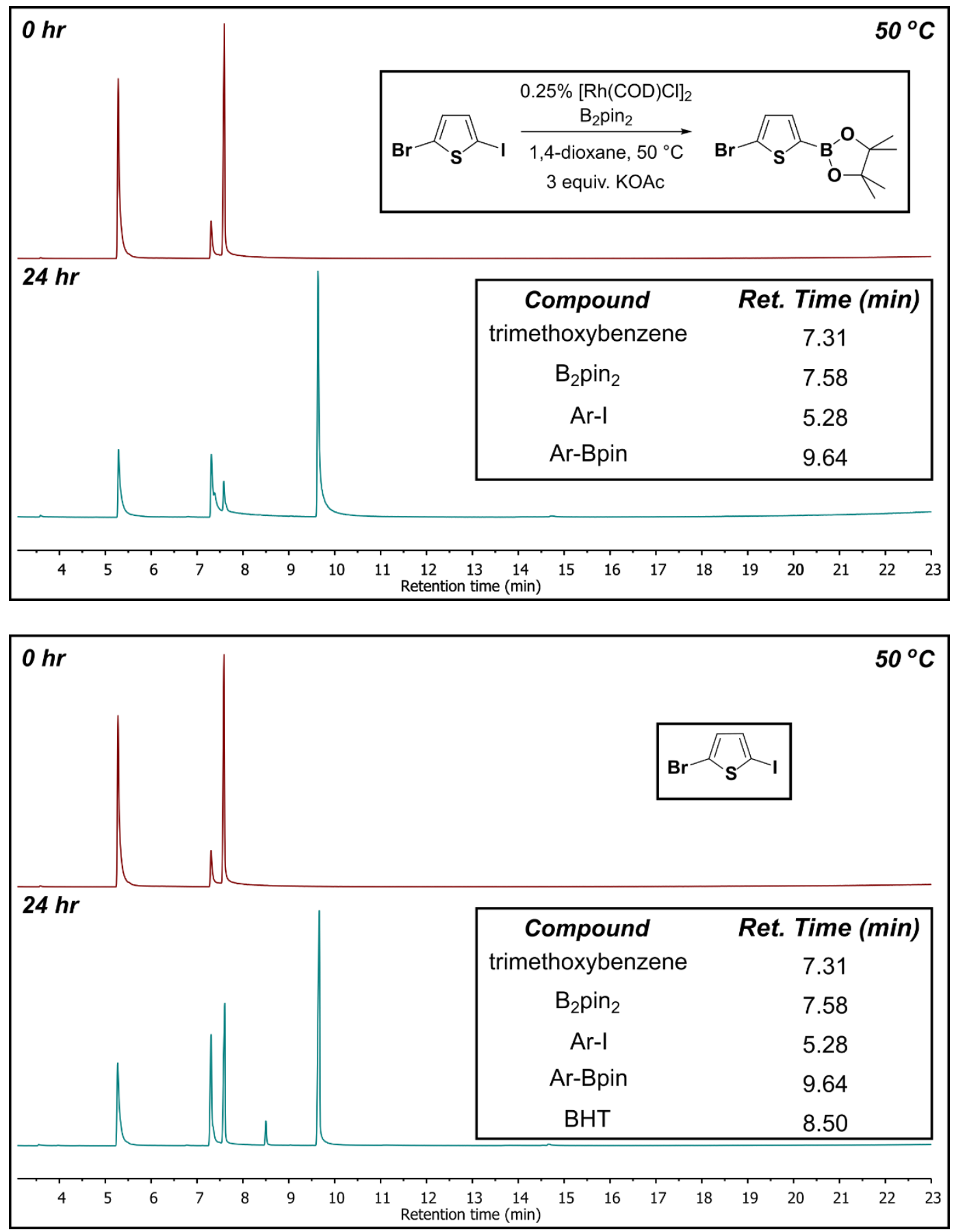

Figure S63. GC-MS spectrum for experiment performed under standard conditions with $0.25 \%$ $[\mathrm{Rh}(\mathrm{COD}) \mathrm{Cl}]_{2}$ (top). GC-MS spectrum for $1 \mathrm{~h}$ time point in experiment performed under standard conditions (bottom). 

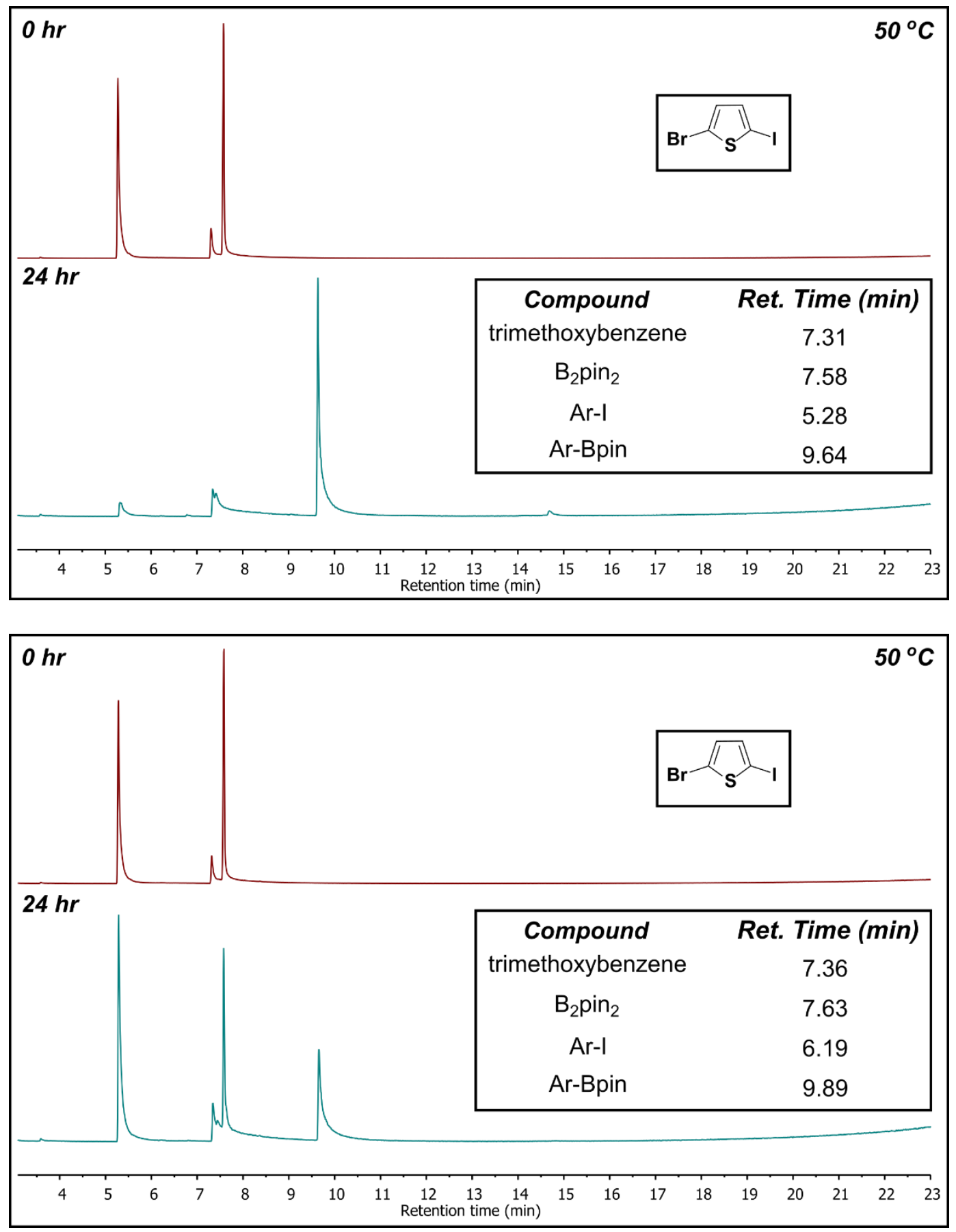

Figure S64. GC-MS spectra for experiments performed under standard conditions with 2 equiv. KOAc (top), and 1 equiv. KOAc (bottom). 